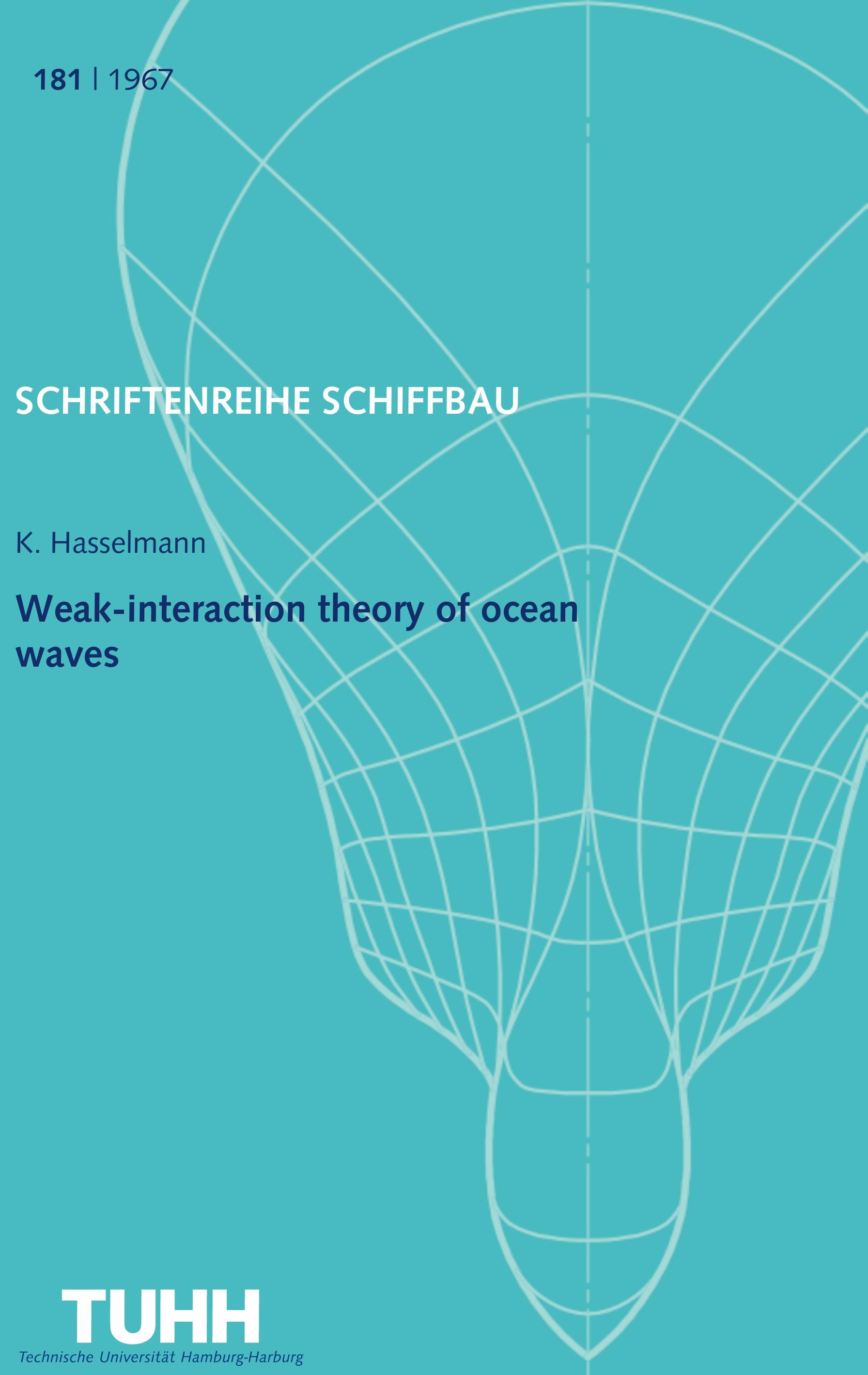




\section{K. Hasselmann, UnIversity of Hamburg}

contents

1. Introauction

2. The raaiation balance

2.1 Representation of the wave field

2.2 The railative transfer equation

2.3 Empirical source functions

3. Theory of weak interactions in random fields

3.1 The interacting fielas

3.2 Interaction diagrams

3.3 The resonant interactions

3.4 The energy transfer

3.5 Transfer àiagrams

3.6 Conservative wave-wave interactions

4. Interactions between gravity waves and the atmosphere

4.1 The lowest-order processes

4.2 The generation of waves by turbulent pressure fluctuations

4.3 The linear interaction with the mean boundary-layer flow

4. 4 Wave-turbulence Interactions

4.5 Non-linear wave-atmosphere interactions

4.6 The pressure spectra

4.7 Comparison with observations 
5. Interactions within the ocean

5.1 The lowest-oraer processes

5.2 The interaction equations

5.3 Interactions with mean currents

5.4 Wave-turbulence interactions

5.5 Strong interactions

5.6 Comparison with observations

6. Conclusions

Appendix. Coupling coefficients 


\title{
Weak-interaction theory of ocean waves
}

\author{
K. Hasselmann
}

\section{Introduction}

Ocean wave research covers a broad range of toplcs incluaing the theoretical analysis of the basic processes of wave growth and decay, semi-empirical methods of wave-forecasting and engineering problems related to the effects of waves on ships, structures and beaches. We shall be concerned here primarliy with the state of the sea as such, rather than the seconaary effects of waves.

The Increased Interest in ocean waves in the past two decades was originally stimulatea by the wave prediction problem. Since aynamical wave theory was virtually nonexistent, wave forecasting evolved for many years as an essentialiy empirical art. However, the latest developments show promise of a stronger interaction with dynamical wave theory, which has made considerable advances in recent years. A general theoretical framework has emerged, enabling a rational aiscussion of both the prediction problem and the dynamical processes determining the local energy balance of the waves. An assessment of the present state of the fleld may be useful before proceeding to the more detalled measurements and computations whlch will be needed to place the theoretical framework on a sounder quant1tative basis.

The flrst prediction methods by Svedrup and Munk (1943, unclassifled 1947) and Suthons (1945) were based on a simplifled parametric description of both wind and wave flelds. Empirical relationships were established between the characteristic parameters of each field. The introduction of a wave spectrum in the prediction methods of Neumann (1953) and Plerson, Neumann and James (1955) represented an Important conceptual advance. However, the reliablilty of these methods and the alternative prediction. formulae suggested by Darbyshire (1955, 1959), Bretschneider (1959), Roll and Fischer (1956), Burling (1959) and others was 
st1ll basically limited by the parametric description of the wind fields, which was retained in the new methods, but was now no longer matched to the more sophisticated description of the wave fleld.

The present forecasting methods use a complete description if both the wind and wave fields and are based on the numerical integration of the radiative transfer equation. The approach was pioneered by Gelc1 and collaborators (cf. Gelc1 et al, 1956, Gelc1 and Cazale, 1962, Fons, 1966) and has been developed In dependently by Baer (1962), Plerson et al, (1966) and Barnett (1966). The source functions used in these methods are st111 largely empirical. However, a closer interaction with dynamical wave theory may be expected in the future. The function. al form of most terms in the source function can now be predict ed theoretically, although extensive measurements and comput ations are still needed to fill in many aetalls。

Dynamical wave theory is the statistical theory of the local interactions of the wave fleld with the atmosphere and ocean. The first significant contributions to this problem were Phillips' (1957) and Miles' (1957) theories of wave generation, which ylelded rigorous transfer expressions for certain aspects of the wave-atmosphere interactions which had been aiscussed previously by Eckart (1953), Jeffreys (1925, 1926) Wuest (1949), Lock (1954) and others. A further contribution was the determination and computation of the nergy transfer due to non-linear wave-wave interactions (Phililps, 1960, Hasselmann $1960,1962,1963 \mathrm{a}, \mathrm{b})$ 。

We shall see in the following that these processes may be regarded as particular applications of a general theory of weak interactions, which ylelds the energy transfer for all expansible interactions between the wave field and the atmosphere and ocean. 
The lowest-order set of transfer expressions for waveatmosphere interactions consists of the Phillips and Miles processes, a non-IInear correction to Miles' process, and wave-turbulence interactions. Present data suggests that the wave-turbulence interactions may be the most important of the four.

The Interactions between waves and the ocean are formally very similar to wave-atmosphere interactions. The lowest-order processes consist of parametric damping by mean currents, scattering by large and medium-scale turbulence and parametric dampling by small-scale turbulence. The last process may be interpreted as an eady viscosity. $9 / \sqrt{A}$ further application of the general interaction theory is the alffusion due to waves, but this will not be considered here.

The major part of this paper w11l be devoted to the development and application of the weak interaction theory. The theory ylelas the source functions for the radiative transfer problem. which will be alscussed briefly in the first section. The interaction and transfer problems are complementary aspects of the complete problem of determining the state of the sea for a glven wind fleld. Although we shall consider only ocean waves in detall, the basic concepts are applicable to all random wave fielas. We shall accordingly present the theory first for an arbitrary set of interacting fields, following Hasselmann $(1966,1967$ a). Since the emphasis is on developing a general approach, we cannot do adequate justice to many specific contributions to the subject; we refer in this respect to the more extensive expositions of Kinsman (1965) and Phililps (1966). 
2. The radiation balance

\subsection{Representation of the wave flela}

Ocean waves are a statistical phenomenon; it 1s meaningful to conslder only average propertles of the wave fleld. In practice, the mean values are determined elther as time or spaclal averages. For theoretical purposes, it is more convenlent to conslier the mean values as averages over a hypothetical ensemble of fields. Our averages will be defined in this latter sense. The two definitions are equivalent if the fleld is elther statistically stationary or homogeneous, 1.e. if all mean quantitles are invarlant under elther time or (horizontal) spacial translations.

To a first approximation, an ocean wave fleld is both stationary and homogeneous. Th1s 1mplles that the aynamical processes changing the state of the fleld are weak, and the fleld may thus be regarded approximately as a superposition of free waves. The fleld can then be represented as a Fourler-Stielties integral (which we write in a more convenient sum notation)

$$
S(x, t)=\sum_{k}\left\{\eta_{k} e^{i(k \cdot x-\sigma t)}+\eta_{k}^{*} e^{-i(k \cdot x-\sigma t)}\right\} \quad \text { (2.1.1) }
$$

where $\zeta$ is the surface displacement (positive upwards) $x_{n}=\left(x_{1}, x_{2}\right)$ is the horizontal coordinate vector $k_{m}=\left(k_{1}, k_{2}\right)$ is the horizontal wave-number vector $\sigma=(g k \tanh k H)^{1 / 2}$ is the frequency of a free surface gravity wave in water of aepth $H$, which we take to be constant, and

$\eta_{k}$ is a random Fourier amplitude. 
For a homogeneous, stationary wave field, the covariance. matrix of the Fourier amplitudes is diagonal,

$$
\begin{aligned}
& \left\langle\eta_{k_{1}} \eta_{m}\right\rangle=0, \\
& \left\langle\eta_{k_{1}} \eta_{k_{2}}\right\rangle=0 \quad \text { for } k_{m} \neq k_{m} \\
& \left\langle\eta_{k} \eta_{k}^{*}\right\rangle=\frac{2}{\rho g} F\left(k_{m}\right) \Delta k_{m}
\end{aligned}
$$

where the cornered parentheses denote ensemble means, $\rho$ is the density of water, 8 is the acceleration of gravity,

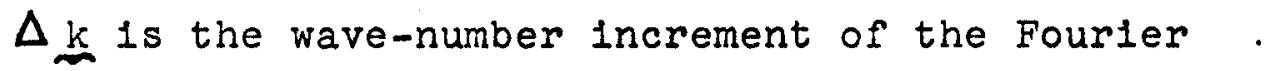
sum anà

$F(k)$ is the (continuous) energy spectrum.

The total wave energy per unlt surface area' is then

$$
E=\rho g\left\langle\zeta^{2}\right\rangle=2 \int F(k) d k
$$

The normalisation of $F$ is that used in the general interaction theory; it differs from the more usual definition by a factor $2 / 98$.

It can be shown that a homogeneous field consisting only of alspersive, free-wave components rapidiy attains a Gaussian state (Hasselmann, 1967 a). In this case the energy spectrum. $F(\mathrm{k})$ completely specifies the fleld statistically.

Since the wave components undergo weak interactions, the Gaussian property, the stationarity and the homogene1ty of the wave fleld are only approximately val1d. The fleld can still be described locally by a spectrum, but this must now be regarded as a slowly varying function of $x$ and $t$, where $\frac{1}{k} \frac{\partial F}{\partial x_{i}} \ll 1, \frac{1}{\sigma} \frac{\partial F}{\partial t} \ll 1$. 


\subsection{The raciative transfer equation}

The evolution of the spectrum $F(k, x, t)$ is determined by the energy balance, or radiative transfer, equation

$$
\frac{D F}{D t} \equiv \frac{\partial F}{\partial t}+\dot{x}_{i} \frac{\partial F}{\partial x_{i}}+\dot{k}_{i} \frac{\partial F}{\partial k_{i}}=S
$$

wheire

$$
\begin{aligned}
& \dot{x}_{i}=\frac{\partial}{\partial k_{i}} \sigma(\underset{m}{x}, \underline{m}) \\
& \dot{k}_{i}=-\frac{\partial}{\partial x_{i}} \sigma(\underline{x}, k)
\end{aligned}
$$

The source function $S$ represents the net transfer of energy to (or from) the spectrum at the wave number $k$ aue to all interaction processes which affect the component $k$. $\frac{D F}{D t}$ is the Lagranglan rate of change of the spectrum relative to a wave group $k$ moving along the path in $x-k$ phase space aeterminea by the Hamiltonian equations (2.2.2) and (2.2.3) (the dot denotes aifferentiation with respect to $t$.). It is assumea that the aepth $H$ is slowly varying, $\frac{1}{k} \frac{\partial H}{\partial x_{i}} \ll 1$, so that the geometrical refraction laws $(2.2 .2)$ and $(2.2 .3)$ are applicable. In deep water, the refraction term $\dot{k}_{i} \frac{\partial F}{\partial k_{i}}$ in (2.2.1) vanishes. Equations $(2.2 .1)$ - (2.2.3) apply for a plane ocean. The corresponaing relations for wave propagation on a sphere are given in Groves and Melcer (1961) and Backus (1962).

Equation (2.2.1) can be derived from geometric ray theory by assuming that the wave-numbers and amplitudes in equation (2.1.1) are slowly varying functions of $x$ and $t$. It can best be unajerstood by regaraing $F(k, x)$ as the energy density in $x-k$ phase space of an ensemble of wave groups. In the case $S=0$, the energy of each wave group remains constant, so that the energy density is proportional to the number density. Equation (2.2.1) can then be inter- 
preted as the continuity equation for the number aensity of wave groups in phase space. The number density, and therefore F, remains constant along a wave-group trajectory, since the flow in phase space defined by equations (2.2.2) and (2.2.3) is incompresslble (Longuet-H1ggins, 1957. The analogy with the Llouvilie theorem was pointea out by Dorrestein, 1960). If the energy of a wave group changes along its propagation path, a source term appears on the right hand side of the equation.

The transfer equation may be expressed in the integral form

$$
F(\underset{m}{k}, \underset{m}{x}, t)=F\left(k_{0}, x_{0}, t\right)+\int_{t_{0}}^{t} S\left(k_{m}^{\prime}, x_{m}^{\prime}, t^{\prime}\right) d t^{\prime}
$$

where $k^{\prime}, x^{\prime}$ and $t$ vary along a wave group trajectory from an inftlal value $k_{0}, x_{0}, t_{0}$ to the point $k, x, t$. Equation

(2.2.4) does not, In general, represent a solution of the transfer problem, since the source function in the integral is a functional of the spectrum.

The major part of this paper will be devoted to determining the source function $S$. We shall show that $S$ is of the general form

$$
S(\underline{k})=s_{1}+s_{2}+s_{3}+s_{4}+s_{1}+s_{2}+s_{3}+s_{4}+s_{5}+s_{6}+s_{7}+\ldots(2.2 .5)
$$

where

$$
\begin{aligned}
& S_{1}=\alpha \\
& S_{2}=\beta F\left(\begin{array}{l}
k \\
m
\end{array}\right) \\
& S_{3}=F(\underset{m}{k}) \int \gamma\left(k, k_{m}^{\prime}\right) F\left(k_{m}^{\prime}\right) d k_{m}^{\prime} \\
& s_{4}=-\delta F(\underline{k})+\int \varepsilon\left(\underline{k}, k^{\prime}\right) F\left(\underline{k}^{\prime}\right) d k^{\prime} \\
& S_{1}^{1}, S_{2}^{\prime}, S_{\frac{1}{3}} \text { and } S_{4}^{\prime} \text { are of the same functional form } \\
& \text { as } S_{1}, S_{2}, S_{3} \text { and } S_{4} \text {, respectively, } \\
& S_{5}=\int\left[T_{1} F\left(k_{m}^{\prime}\right) F\left(k_{m}^{\prime \prime}\right) F\left(k_{m}-k_{m}^{\prime}-k_{m}^{\prime \prime}\right)-T_{2} F\left(k_{m}\right) F\left(k_{m}^{\prime}\right) F\left(k_{m}^{\prime \prime}\right)\right] d k^{\prime} d k_{m}^{\prime \prime} \\
& S_{\sigma}=-x_{i j} k_{i} k_{j} F\left(k_{m}\right) \\
& \mathrm{S}_{7} \text { represents the as-yet-unknown dissipation due to } \\
& \text { wave breaking. }
\end{aligned}
$$


We have expressed only the dependence on the wave spectrum $F$ explicitly. The coefficients $\alpha, \beta$ etc. aepend in a known manner on the properties of the other interacting fields.

The source function $S_{1}$ represents the constant energy transfer to the wave fiela through turbulent atmospheric pressure fluctuations (Eckart, 1953, Phililps, 1957); $\mathrm{S}_{2}$ corresponas to Miles' (1957) unstable coupling mechanism between the wave fleld and the mean boundary layer flow; $S_{3}$ is a non-linear correction of Miles' theory, and $S_{4}$ represents the energy transfer due to wave-turbulence Interactions (Hasselmann, 1967 a).

The processes $S_{1}$ to $S_{4}$ represent the complete set of lowest oraer transfer expressions due to interactions with the atmosphere. With the possible exception of $\mathrm{S}_{2}$, all are probably 1mportant in varlous frequency ranges and stages of development of a wina-generated sea.

The interaction of waves with mean currents and turbulence in the ocean are formally very similar to the interaction with the mean flow and turbulence in the atmosphere. The corresponding transfer expressions $S_{1}$ to $S_{4}$ are of the same functional form as the transfer expressions $S_{1}$ to $S_{4}$. However, $S_{1}^{\prime}$ and $S_{2}^{\prime}$ are normally negligible.

$S_{5}$ represents the energy transfer due to non-linear wave-wave Interactions (Hasselmann, 1960, 1962). The energy transfer has been computed for typical wave spectra and was found to be not inconsistent with spectra observed to the lee of generating areas (Snodgrass et al, 1966).

$S_{6}$ represents the alssipation in shallow water aue to turbulent bottom friction (Hasselmann and Collins, 1967). $\nu_{i j}$ is a known functional of the wave spectrum. The expression is basea on a quaoratic friction law. In contrast to the processes mentioned above; it cannot be derived from the weak-interaction theory, since the friction law is non-expansible. 
Another example of a weak, but probably non-expansible process is the energy loss $S_{7}$ aue to wave-breaking, which is still undetermined.

We note that with the exception of $S_{1}, S_{2}$ and $S_{1}, S_{2}$, all source functions depend on the entire wave spectrum, and not only on the component $\mathrm{k}$. The source functions $\mathrm{s}_{4}, \mathrm{~s}_{4}, \mathrm{~s}_{5}, \mathrm{~s}_{6}$ and $\mathrm{S}_{7}$ are furthermore non-linear in $F$. The first property 1 s common to many transfer problems (c.f. Chanaresekhar, 1960, Kourganoff, 1963). It lmplies that all wave components of the flela are couplea. It is not possible to aetermine the spectrum at any point $P$ in the ocean by 1ntegrating the source function along only? torles which terminate in $\mathrm{P}$; the spectrum must be determined simultaneousiy in the entire region of the ocean in which $S$ is non-zero. The numerical integration of the transfer equation nontheless lies within the capacity of present-äay difital computers, ano several programs for prealcting areal wave spectral distributions are already in operation. 


\subsection{Empirical source functions}

Thesource functions acopted in present wave forecasting programs are largely empirical.

In the latest version of their method DSA (a1stribution spectroangulaire) Gelc1 ana Cazalé (1966) Introduce a source function

$$
S_{D S A}=\alpha-A \sigma^{4} F(\underset{m}{k}) \int(B-C \cos \varphi) F\left(k_{m}^{\prime}\right) d k^{\prime}
$$

where $\alpha$ is a function of $k$ and the wind velocity $U, \varphi$ is the angle between $k$ and $\underset{\sim}{ }$ and $A, B$ and $C$ are constants. $S_{D S A}$ is of the form $S_{1}+S_{3}$. However, the non-linear term is introduced as the energy loss aue to wave breaking, rather than a non-Iinear correction to Miles' theory. A Iinear term $\mathrm{S}_{2}$ corresponding to Miles' theory is not incluaied.

Plerson, Tick and Baer (1966) suggest a source function

$$
S_{P T B}= \begin{cases}{\left[\alpha\left(1-\gamma^{2}\right)^{1 / 2}+\beta E(k)\right]\left(1-\gamma^{2}\right) s(k, \varphi)} & \text { for } F<F_{\infty},|\varphi|<\pi / 2 \\ 0 & \text { for } F>F_{\infty},|\varphi|<\pi / 2 \\ \Phi & \text { for }|\varphi|>\pi / 2\end{cases}
$$

In which

$$
\begin{aligned}
& \alpha, \beta \text { are functions of } k \text { and } U, \\
& E(k)=\int_{-\pi}^{+\pi} F(k) k d \varphi \text { is the one-aimensional spectrum, } \\
& s(k, \varphi) \text { is an empirical angular spreading factor, with } \\
& \int_{-\pi}^{+\pi} s(k, \varphi) d \varphi=1
\end{aligned}
$$

$\Phi$ is a alssipation function whlch acts only for waves traveling against the wind,

$$
\gamma=\frac{E(k)}{E_{\infty}(k)}
$$


ana $F_{\infty}(k, U), E_{\infty}(K, U)$ are empirical fully aeveloped spectra taken from Plerson and Moskowitz (1964). As a function of frequency, the one-almensional Pierson-Moskowitz spectrum is given by

$$
E_{\infty}(\sigma)=\alpha g^{2} \sigma^{-5} \exp \left\{-\beta\left(\sigma_{1} / \sigma\right)^{4}\right\}
$$

where $\sigma_{1}=g / u, \alpha=0.0081$ and $\beta=0.74$

The form of the source function (2.3.2) ensures that the waves tend to a fully-aevelopea Plerson-Moskowitz spectrum in a uniform wind fleld. For small values of the spectrum, the growth of the one-almensional spectrum $E(K)$ (but not $F(\underline{K})$ ) is in accoraance with a combinea Phillips-Miles mechanism, $S=S_{1}+S_{2}$. The functions $\alpha$ and $\beta$ were determined from the field measurements of Snyajer and Cox (1966) (section 4.6).

Barnett (1966) introduces a source function

$$
S_{B}=\alpha+\beta F(k)(1-\Phi)+S_{5}
$$

In wh1ch $\alpha$ ana $\beta$ are functions of $\mathrm{k}$ and

$$
\begin{aligned}
& \Phi=a \exp \{-b(R-F) / F\} \quad \text { with } a, b \text { constant, and } \\
& R=\gamma k^{-4} s(\varphi) \text { corresponàs to Ph1llips' (1958) universal }
\end{aligned}
$$
equilibrium spectrum ( $\operatorname{section} 5.5$ ), with $\gamma=\operatorname{const,} s(\varphi)=\frac{8}{3 \pi} \cos ^{4} \varphi$. For computational convenience, the wave-wave scattering integral $S_{5}$ was approximatea by a parametrical expression. $\alpha$ and $\beta$ were determined from the measurements of Snyder and Cox (1966).

The source function $S_{B}$ is of the general form

$$
S=s_{1}+s_{2}+s_{5}+s_{7}
$$

$\left(\right.$ w1th $\left.s_{7}=-\left(S_{1}+S_{2}\right) \Phi\right)$

suggestiea by Hasselmann (1960) on the basis of then existing 
theories of wave generation and wave-wave scattering.

A similar source function, with $S_{5}=0, s_{7}=-\left(s_{1}+s_{2}\right)\left(\frac{E}{E_{\infty}}\right)^{2}$ has been consiciered in the one-dimensional case by Inoue $(1966)$.

The alscrepancy between the varlous emplrical source functions is consiaerable. It points to the aificulty of making sufficiently detallea, conclusive measurements of wave growth and decay in the ocean. A considerable gap exists still between our understanilng of the basic aynamical processes, as indicated by the general form of the theoretical source function (2.2.5), and the application of this knowleage to the forecasting problem. The affflculty is that although theory can furnish the transfer expressions for wave interactions with other fielas, the transfer rates depend on the detallea statistical properties of the interacting fielos, which can be determined only experimentally. To aetermine the source functions reliably, measurements of wave growth and decay need to be combined with detalled measurements of the interacting fielos. Although the present source functions w1ll almost certainly be modified in the light of future experiments, the aevelopment of the radiative transfer method is nontheless an important first step towards a rational treatment of the wave prealction problem. 


\section{Theory of weak interactions in ranaiom fields}

The principal goal of dynamical wave theory is the aetermination of the theoretical source functions summarized in equation (2.2.5). The problem may be âiviced into two:

(1) the determination of the coupling coefficients charact,erizing the interactions between the wave field and its physical environment, and

(11) the evaluation of the energy transfer due to these interactions.

The first problem involves the detalled analysis of the non-linear equations of motion of the interacting fields. We shall consider this in later sections.

The second problem may be treated without. specific reference to the type of interacting flela. The theory applies to any system Involving weak interactions between wave flelas and other random fields, e.g. the generation of sound by turbulence, the scattering of light and sound in the atmosphere, interactions between Rossby waves and currents, plasma-wave interactions, etc.

In this section we shall develop the theory in a general form, considering later its specific application to ocean waves.

The theory is as a rather straightforwara extension of the analysis of conservative wave-wave interactions, which was first considered in aetall for the case of non-Iinear lattice vibrations in solias by Pelerls (1929), and is well known in many branches of physics, particularly in quantum fiela scattering theory.

One of the main difficulties which arise is keeping track of the numerous terms occuring in the perturbation expansion of the flelos and ordering the various transfer expressions associated with different term combinations. Here, diagram notations are useful. In the case of conservative wave-wave interactions, both the perturbation expansion and the transfer expressions can be summarlzed by a single set of diagrams, which may be interpreted 
In a particle picture anà correspona to Feynman diagrams in quantum field theory (Hasselmann, 1966). The general case of non-conservative interactions with non-wave flelds is more complicated. Two types of alagrams are needea: interaction diagrams, to describe the perturbation expansion of the fiela amplitudes, and transfer diagrams, to summarlze the energytransfer expressions (Hasselmann, 1967 a).

\subsection{The interacting flelàs}

Consiaer a set of weakly interacting flelas consisting of wave flelás, denotea by inälces $\nu$, anà non-wave (external) flelás $\mu$. We shall be concernea only with interactions which affect.the wave flelas. We can then alstingulsh between two types of interaction: wave-wave Interactions, involving wave components only ana external interactions, Involving both wave components and external-fielö components. Interactions between external fielós only have no effect on the wave flelas.

The set of all wave fields will be termed the wave-fiela system. We assume that the wave-wave interactions conserve energy and momentum of the wave-field system. (Non-conservative wave-wave interactions can be treated formally as external interactions.)

The physical system is assumed to be homogeneous in $x$, where $x$ is elther a two- or three-aimensional coordinate vector ( 1 . the case of the ocean, $x=\left(x_{1}, x_{2}\right)$ is the horizontal coorainate vector). We assume further that all flelds are random and statisticaliy homogeneous with respect to $\mathrm{x}$.

In the IInear approximation, let the wave-field system consist of a set of normal mocies $\varphi_{k}^{\nu} \exp \left\{i\left(k, x \pm \omega_{k}^{\nu} t\right)\right\}$

where $\varphi_{k}^{\nu}$ is an elgenfunction (for three-aimensional $x_{m} \varphi_{k}^{\nu}$ may aegenerate to an elgenvector or simply a constant) and $\omega_{k}^{\nu}$ is the elgenfrequency. We assume that the amplitudes $q_{k}^{\nu}$ of the elgenfunctions represent a complete set of coorainates for. the wave-fiela system. 
$-15-$

Let the evolution of the wave-field system, excluding the non-conservative external interactions, be specified by a Lagrangian

$$
\mathcal{L}\left(\dot{q}_{\underline{m}}^{\nu}, \dot{q}_{\underline{k}}^{\nu}\right)=\mathcal{L}_{2}+\mathcal{L}_{\text {int }}
$$

where

$$
\mathcal{L}_{2}=\sum_{k, \nu} \frac{1}{2}\left(\dot{q}_{m}^{\nu} \dot{q}_{-k}^{\nu}-\left(\omega_{k}^{\nu}\right)^{2} q_{k}^{\nu} q_{-k}^{\nu}\right)
$$

is the free-fielo Lagrangian of the linear system and $I_{\text {int }}$ is the wave-wave interaction Lagrangian. The harmonic-osc1liator form of $\mathrm{I}_{2}$ is uniquely determined, except for an arbitrary normalisation factor, by the form of the normal modes (symmetrical propagation characteristics in the positive and negative $\mathrm{k}$ direction) and the homogeneity conation.

It 1 s convenient to transform to canonical variables $q_{k}^{\nu}, p_{k}^{\nu}$,

$$
p_{k}^{\nu}=\frac{\partial \mathcal{L}}{\partial \dot{q}_{k}^{\nu}}, H\left(p_{k}^{\nu}, q_{k}^{\nu}\right)=\sum_{k, \nu} p_{k}^{\nu} \dot{q}_{k}^{\nu}-\mathcal{L}
$$

and then to standard wave variables $a_{k}^{\nu}, a_{k}^{-\nu}$ defined by

$$
\begin{aligned}
& a_{k}^{\nu}=\frac{1}{\sqrt{2}}\left(p_{-k}^{\nu}-i \omega_{k}^{\nu} q_{k}^{\nu}\right) \\
& a_{k}^{-\nu}=\frac{1}{\sqrt{2}}\left(p_{-k}^{\nu}+i \omega_{k}^{\nu} q_{k}^{\nu}\right)
\end{aligned}
$$

$(3.1 .1)$

The equations of motion become in these variables

$$
\ddot{a}_{k}^{v}=-i \omega_{k}^{\nu} \frac{\partial H}{\partial \underline{a}_{-k}^{-\nu}}
$$

where for negative indices the frequency is defined by 


$$
\omega_{k}^{-\nu}=-\omega_{k}^{\nu}
$$

The free-fiela Hamiltonian is given by

$$
H_{2}=\sum_{k, \nu \geqslant 0} \frac{1}{2} a_{k}^{\nu} a_{-k}^{-\nu}
$$

The linear solution of equation (3.1.2) for $\mathrm{H}=\mathrm{H}_{2}$ is

$$
a_{k}^{\nu}=\alpha_{k}^{\nu} e^{-i \omega_{k}^{\nu} t}, \alpha_{k}^{\nu} \text { constant } \nu \geqslant 0
$$

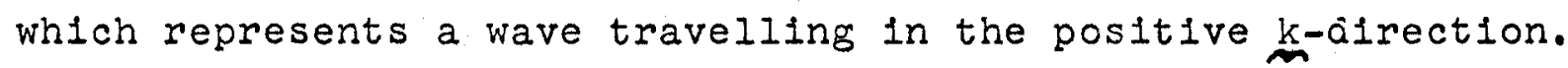

We assume now that the interaction Hamiltonian can be regaried as a small perturbation of the complete Hamiltonlan $\mathrm{H}=\mathrm{H}_{2}+\mathrm{H}_{\text {int }}$, $\mathrm{H}_{\text {Int }} \ll \mathrm{H}_{2}$. We assume further that $\mathrm{H}_{\text {int }}$ can be expancied in a. Taylor serles,

$$
\mathrm{H}_{\text {Int }}=\mathrm{H}_{3}+\mathrm{H}_{4}+\cdots
$$

where

$$
H_{n}=\sum_{k_{j}, \nu_{j} \geqslant 0} D_{k_{1} \cdots k_{n}}^{\nu_{1} \cdots v_{n}} a_{k_{1}}^{\nu_{1}} \cdots a_{k_{n}}^{\nu_{n}}
$$

and $D_{k_{1} \cdots k_{n}}^{v_{1} \cdots \nu_{n}}$ is a constant coupling coefficient.

The conaltion $\mathrm{H}_{\text {Int }} \ll \mathrm{H}_{2}$ is Implicit in the definition of a , wave flela. If $\mathrm{H}_{\text {int }}=O\left(\mathrm{H}_{2}\right)$, it is no longer meaningful to speak of normal modes, dispersion relations, etc. The expansibility assumption is valia in most cases, but not always. (For example, white-cappling 
The Hamiltonian represents the total energy of the wave-fielo system per unit $x$ and is therefore real. The reality of $\mathrm{H}_{2}$ Implies

$$
a_{k}^{\nu}=\left(a_{-k}^{-\nu}\right)^{*}
$$

The reality of $H_{n}$, together with the condition $(3.1 .6)$, yields the relations

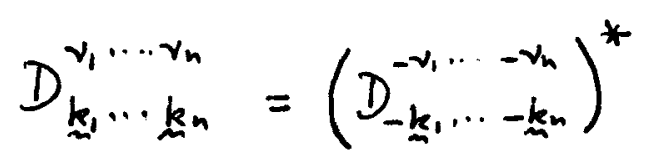

for the interaction coefficients. A second conaition for the interaction coefficlents follows from the invariance of the physlcal system under horizontal translations,

$$
D_{k_{1} \cdots k_{n}}^{\nu_{1} \cdots v_{n}}=0 \text { for } k_{1}+\cdots+k_{n} \neq 0
$$

For uncier a translation $\widetilde{x}=x+\xi{ }_{m}$, the wave components transform to $\tilde{a}_{k}=a_{k}^{v} e^{-i k} \sum_{n}$. The coupling coefficients therefore transform to $\tilde{D}_{k_{1}}^{\gamma_{1} \cdots \cdots k_{n}}=D_{k_{1} \cdots k_{n}}^{v_{1} \cdots v_{n}} \exp \left\{i\left(k_{1}+\cdots+k_{n}\right) \xi_{n}\right\}$ which is invariant only unaer condition (3.1.8). We note further that the coefficients $D_{k_{1}}^{\gamma_{1} \cdots v_{n}}$ can be defineal to symmetrical with respect to the indices $1, \ldots n$.

The equations of motion (3.1.2) for the Hamiltonian (3.1.4) become

$$
\begin{aligned}
& \ddot{a}_{m}^{\nu}+i \omega_{k_{m}}^{\nu} a_{k}^{\nu}=-3 i \omega_{m}^{\nu} \sum D_{-k_{m} k_{1} k_{2}}^{-\nu \gamma_{1} \nu_{2}} a_{k_{1}}^{\gamma_{1}} a_{k_{2}}^{\gamma_{2}}-\cdots \cdot
\end{aligned}
$$

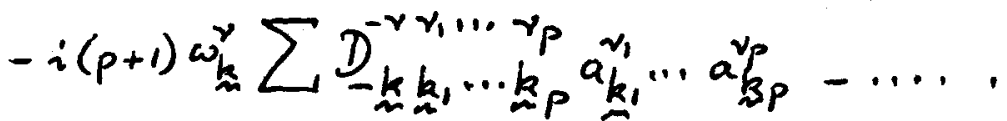


To incluae external interaction within the same framework as wave-wave interactions, we assume now that the external flelds can be described by a set of varlables

$$
b_{k}^{\mu}(\lambda, t)=\beta_{k}^{\mu}(\lambda) e^{-i \omega_{k}^{\mu} t}
$$

In analogy with the representation (3.1.3) of the free wave fields.

The superscript $\mu$ is a combination of a alscrete inaex specifying the external fielo and an aditional varlable which determines the frequency $w_{k}^{\mu}$. In the case of wave flelas, the frequency $\pm \omega_{k}$ is uniquely determined by a discrete inaex $\nu$ which speclfies the wave field and the appropriate alspersion curve. Since the frequencles of the external flelds can vary continuously

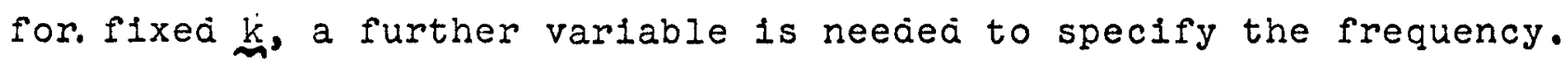
To maintain the analogy with the wave-component notation, we choose $\mu$ such that $\omega_{k}^{\mu}>0$ for $\mu>0$ and

$$
\omega_{k}^{-\mu}=-\omega_{k}^{\mu}
$$

The varlable $\lambda$ represents the set of all further parameters specifying the external flelo.

The reality condition corresponaing to equation $(3.1 .6)$ is

$$
b_{k}^{\mu}(\lambda)=\left(\underline{b}_{-k}^{-\mu}(\lambda)\right)^{*}
$$

As example, consider the turbulence flelas in the ocean (w) or the atmospheric boundary layer (a). We assume that the fielas are statistically stationary and homogeneous with respect to the horizontal coorilinate vector $x_{m}=\left(x_{1}, x_{2}\right)$. The fluctuating velocity fielás $u_{i}^{\gamma}\left(x_{m}, z, t\right)$, where $\gamma=w$ ora, $z=$ vertical coorälnate, may then be expressea as Fourier sums (FourlerStleitjes integrals) 
$-19-$

$$
u_{i}^{\gamma}(x, z, t)=\sum_{k, \omega} u_{i, k, \omega}^{\gamma}(z) e^{i(k, x+\omega t)}
$$

If we define

$$
b_{k}^{\mu}(\lambda, t)=\beta_{k}^{\mu}(\lambda) e^{-i \omega_{k}^{\mu} t} \equiv u_{i, k, \omega}^{\gamma}(z)
$$

and use the notation

$$
\mu \equiv(\gamma,-\omega), \omega_{k}^{\mu}=-\omega, \lambda \equiv(i, z)
$$

this may be written

$$
u_{i}^{\gamma}(x, z, t)=\sum b_{m}^{\mu}(\lambda, t) e^{i k \cdot x}
$$

Using this notation, we may include interactions with external fields in equation (3.1.9) simply by adding further terms to the right hand side,

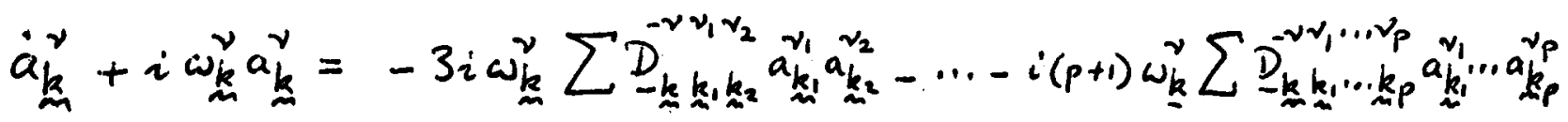

$$
\begin{aligned}
& -2 i \omega_{k}^{\nu}\left(\sum_{\nu_{1}} E_{-k v_{m}}^{-\nu \nu_{1}} a_{k_{1}}^{\nu_{1}}+\sum_{\mu} E_{-k}^{-\nu \mu} b_{k}^{\mu}\right)-\cdots \cdot
\end{aligned}
$$

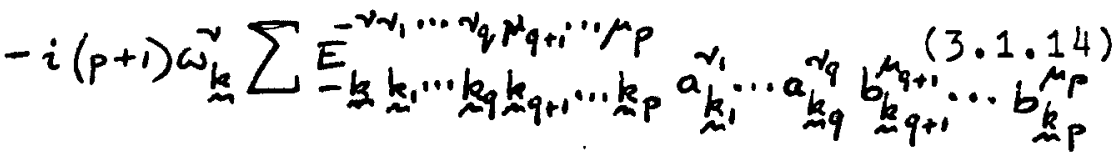


The external coupling coefficlents $E_{-k_{1}}^{-\nu \nu_{1} \cdots \nu_{q} \mu_{q} \mu_{q+1} \cdots k_{q+1} \cdots k_{p}}$ satisfy the same reality and homogeneity conditions $(3.1 .7)$ and $(3.1 .8)$ as the internal coupling coefficients. However, since they are not derlved from a Hamiltonian, they are not symmetrical with respect to all permutations of the indices. Besides the indices shown, the coefflclents aepend on the variables $\lambda_{j}$, which are also included in the summations in equation (3.1.14).

We note that the external interactions include non-conservative interactions between wave components, and that the lowest orderterms are linear.

Equations (3.1.14) represent the equations of motion for the wave flelds only. The external fielas are regarajo as given. The back-interaction of the wave fielas on the external fielas is assumea to be either negligible or already incluaed in the definition of the coupling coefficlents $E$. In the latter case, the external field components $b_{k}^{\mu}$ refer to the unaisturbed external flelds.

\subsection{Interaction diagrams}

Since the interactions are weak, we can construct solutions of equation ( 3.1 .14 ) by perturbation or 1teration methoas. For a general discussion, the iteration method is more convenient, as the perturbation parameters need not be specified.

Writing equation (3.1.14) symbolically in the form

$$
I[a]=N[a, b]
$$

the n'th iteration $n^{a_{k}^{\nu}}$ is given by

$$
n^{a}=L^{-1} N[n-1, b]
$$


$-21-$

The first order solution, $a_{k}^{\nu}$ is the free field solution, equation (3.1.3).

Explicitly, equation $(3.2 .1)$ is given by

$$
\begin{aligned}
& n^{a_{k}^{\nu}}(t)=\alpha_{k}^{\nu} e^{-i \omega_{k}^{\nu} t}+\int_{0}^{t} d t^{\prime} e^{-i \omega_{k}^{\nu}\left(t-t^{\prime}\right)} x
\end{aligned}
$$

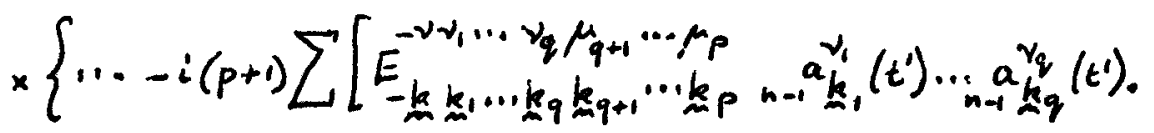

$$
\begin{aligned}
& \left.\left.\cdot b_{k_{q+1}}^{\mu q+1}\left(t^{\prime}\right) \ldots b_{k_{p}}^{\mu p}\left(t^{\prime}\right)\right] \ldots\right\} \text {. }
\end{aligned}
$$

where $\quad \alpha_{k}^{\gamma}=a_{\underline{m}}^{\gamma}(t=0)$.

The structure of the solutions rapidly becomes complicated as $n$ increases and 1 is convenient to introduce a simpler notation in terms of interaction diagrams. To this end, let us represent the field components $a_{k}^{\gamma}, b_{k}^{\mu}$ with $\nu_{2} \mu>0$ by arrows equal to the wave-number vectors $\mathrm{k}$. Similarly, let the

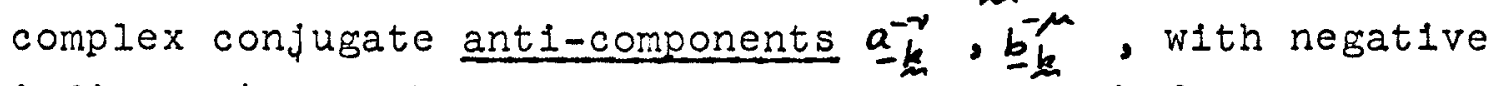
indices $-\nu,-\mu$, be represented by cross-stroked arrows equal. to $\mathrm{k}$. The sign convention is chosen such that the arrows point in the propagation directions for both components $\nu, \mu$ and ant $1-$ components $\bar{\nu}, \bar{\mu}$.

We may now represent the general term in the square parentheses in equation (3.2.2) by $p$ arrows ( $q$ components ${ }_{n-1}^{a_{k j}^{r}}$ and $p-q$ components $b_{k j}^{j}$ ) entering a vertex ana a single arrow (the contribution of the term $[\ldots .$.$] to { }_{n} a_{k}^{\nu}$ ) leaving the vertex. The linear term $\alpha_{k}^{\nu} e^{-i \omega_{k}^{\nu} t}=a_{k}^{\nu}$ is represented simply by an arrow. The complete expression (3.2.2) is then given by the linear diagram and the set of all possible diagrams with one vertex ana one outgoing component. 
The Ingolng components ${ }_{n-1} a_{k}^{\nu_{j}}$ in these diagrams can be reaucea by further diagrams to components ${ }_{n-2} a_{k}^{\nu}$, the components ${ }_{n-2} a_{k}^{\nu}$ to ${ }_{n-3} a_{k}^{\nu}$, and so forth to the components ,$a_{k}^{\nu}$, which are given by the initial conaitions. One obtains in this manner branch diagrams contracting through a series of vertices from à number of input components, $a_{k_{j}}^{\gamma_{j}}, b_{\mathbf{j}_{j} \nu}$ to a single output component. The $n^{\text {th }}$ order iteration $n^{2}$. Is represented by the set of all interaction diagrams containing not more than $n-1$ vertices.

Each vertex of a aigram is associated with a coupling coefficient and a number of field components. In applications, these are normally characterized by certain small parameters. The order of an interaction diagram with respect to these parameters is immediately apparent. The representation of the iteration. solutions as a perturbation series involves oniy thetordering of alagrams with respect to the parameters chosen; the alagrams themselves are indepenaent of the representation of the solution.

\subsection{The resonant interaction}

The forcing terms in equation (3.1.14) consist of products of exponentials $e^{ \pm 1 \omega t}$. If the resultant sum frequency is equal to the elgenfrequency $\omega_{\vec{k}}^{\nu}$, the response is non-stationary. An interaction alagram rêpresents a resonant interaction if

$$
\sum_{\gamma} s_{\gamma} \omega_{\gamma}=s_{\nu} \omega_{\gamma}
$$

where $\omega_{j}>0, s_{j}= \begin{cases}+1 & \text { for compowents } \\ -1 & \text { for anti-components }\end{cases}$

and the sum is taken over all ingoing components $\gamma$.

Further resonances occur if equation (3.3.1) holds for any subalagram within an interaction alagram. 
The homogeneity conaition (3.1.8) ylelas an analogous relation for the wave numbers,

$$
\sum_{\gamma} s_{\gamma} k_{\gamma \gamma}=s_{\nu} k_{\nu}
$$

which is valid for all aiagrams.

We shall alstingulsh between resonant (free-wave) and forced (virtual) components in a alagram by full anc dottea arrows, respectively.

Forcea components represent small moaifications of the free fielas. They are normally of secondary physical interest. However, the analysis of higher oraer non Gaussian properties due to the forced components) can yleld important information about the coupling coefflclents(cfiasselmann et al, 1963).

The resonant interactions lead to a continuous redistribution of energy between the interacting components. Our primary goal will be to determine the source functions characterizing this energy flux.

\subsection{The energy transfer}

Let us consider first the effect of the interactions on the statistical properties of the flelas.

It can be shown that free, aispersive wave flelōs asymptotically become Gaussian, stationary ana statistically inaepenaient (Hasselmann, 1967 a). These properties hold in the coarsegrained sense, assuming that all mean values can be aetermined only with an arbitrary large, but finite spectral resolution. In practice, this is always the case.

The flelos are then completely determinea statistically by the set of time-indepencient energy spectra 


$$
F_{k}^{\nu}=\frac{1}{2}\left\langle a_{k}^{\nu} \underline{a}_{k}^{-\nu}\right\rangle
$$

The total energy of the wave-fleld system is accoraingly

$$
H_{2}=\sum_{k, \nu \geqslant 0} F_{k}^{\nu}=\text { const }
$$

We may expect the interactions to modify this simple picture In two respects: firstly, the non-linear distortion of the fleld due to the forced-interaction components will give rise to non-Gaussian statistical properties; seconoly, the resonant interactions w1ll destroy the stationarity of the system, proaucing, in particular, a continuous reaistribution of energy within the spectrum. We are concerned here with the latter . effect.

It is not immealately apparent that the energy transfer can be conslaerea separately from the non-Gaussian aistortion. For example, In the case of conservative wave-wave interactions, the total energy $\mathrm{H}=\mathrm{H}_{2}+\mathrm{H}_{\text {Int }}$ consists of the total spectral energy $\mathrm{H}_{2}$ and the energy $\mathrm{H}_{\text {int }}$ associated with the field aistortions. It is an important result of weak-interaction theory that to lowest order the resonant interactions affect only the spectral alstributions, and not the partition of energy between $\mathrm{H}_{2}$ and $\mathrm{H}_{\text {Int }}$; thus the total spectral energy is conserved, $\frac{\partial \mathrm{H}_{2}}{\partial t}=0$. The statistical properties of the distortion fiela are given to lowest orier as stationary functions of the spectral distributions. As the spectral distributions vary, there is therefore a seconaary, lower oraer redistribution of energy between $\mathrm{H}_{2}$ and $\mathrm{H}_{\text {int. The same situation applies if energy }}$ is Introduced or withorawn by external interactions; to first order, the energy transfer affects only the energy spectra assoclated with $\mathrm{H}_{2}$. 
The redistribution of energy can be determined by expanding $F_{k}$ in terms of the various alagram contributions to $a_{k}^{\nu}$ and a which grow linearly in $t$. The secular terms can then be rewritten as the rate of change of a slowiy varying spectrum. The analysis is well known from various scattering problems in solia state ana quantum flelò theory. A derivation in the present context is given in Hasselmann (1967 a). We inalcate here only the structure of the analysis in terms of the interaction alagrams assoclated with typlcal transfer terms. Since $\mathrm{F}_{\mathrm{K}}^{\nu}$, is quadratic, each term involves two alagrams representing the relevant contributions to $a_{k}^{\nu}$ and $a_{-k}^{-\nu}$, respectively.

The net energy transfer, or source function $S$, consists of a number of contributions, which are listed belpw. We return to the continuous spectrum notation $F_{\nu}(\mathrm{k})=\frac{F_{k}}{\Delta k_{m}}$, where $\Delta \mathrm{k}$ is the wave-number increment of the Fourier sum. The source functions represent the energy transfer to the normal mode $\nu, S_{. .}^{\gamma}(k)=\frac{D}{D t} E(k)$

The linear interactions yield the transfer expressions

$$
S_{\nu}^{\nu}(k)=T_{\nu}^{\nu}(k) F_{\nu}(\underline{k})
$$

and

$$
S_{(\mu \nu)}^{\nabla}(k)=\int T_{\mu \rightarrow \nu}^{\nu}\left(k_{\mu}, \lambda, \lambda^{\prime}\right) F_{\mu}\left(k, \lambda, \lambda^{\prime}\right) \delta\left(\omega_{k}^{\nu}-\omega_{k}^{\mu}\right) d \lambda d \lambda^{\prime}
$$

where

$$
T_{\nu}^{\nu}(k)=4 \omega_{m}^{\nu} \mathcal{F}_{m}\left(E_{-k v}^{-\nu \nu}\right)
$$


$-26-$

$$
T_{\mu \rightarrow \nu}^{\nu}\left(k, \lambda, \lambda^{\prime}\right)=8 \pi\left(\omega_{k}^{\nu}\right)^{2} E_{-k k}^{-\nu \mu}(\lambda)\left(E_{-k \mu}^{\nu \mu}\left(\lambda_{\mu}^{\prime}\right)\right)^{*}
$$

ana

$$
F_{\mu}\left(\underline{k}, \lambda, \lambda^{\prime}\right)=\frac{\left\langle b_{k}^{m}(\lambda) b_{k}^{-r}\left(\lambda^{\prime}\right)\right\rangle}{\Delta \underline{\underline{k}}}
$$

is the external field spectrum.

The Index notation for source functions $S$ and transfer functions $T$ refer to the transfer alagrams introduced in the next section. The relevant interaction diagram pairs are shown in figure 1. We note that although both processes are linear, the transfer rates $(3.4 .2)$ and $(3.4 .3)$ are of different order with respect to the coupling coefficients.

The nonlinear interactions yield transfer integrals involving quadratic and higher products or spectra. We give here only the quadratic expressions.

The wave-wave interactions lead to four transfer expressions which in the general, non-conservative case are given by

$$
\begin{aligned}
S_{\left(\nu_{1} \nu_{2} \bar{\gamma}\right)}^{\bar{\nu}}= & \int\left(T_{\nu_{1} \nu_{2} \rightarrow \nu}^{\nu} F_{\gamma_{1}} F_{\nu_{2}}-T_{\nu \bar{v}_{1} \rightarrow \nu_{2}}^{\nu} F_{\gamma} F_{\nu_{1}}-T_{\nu \bar{v}_{2} \rightarrow \nu_{1}}^{\nu} F_{\nu} F_{\nu_{2}}\right) . \\
& . \delta\left(\underline{k}_{1}+k_{2}-k_{2}\right) \delta\left(\omega_{k_{1}}^{\nu}+\omega_{k_{2}}^{\nu_{2}}-\omega_{\underline{k}}^{\nu}\right) d k_{1} d k_{2}
\end{aligned}
$$

$$
\begin{aligned}
& S_{\left(\gamma_{1}, \bar{\nu} \bar{\nu}\right)}^{\bar{\gamma}}=\int\left(T_{\nu, \bar{\nu}_{2} \rightarrow \nu}^{\nu} F_{\nu_{1}} F_{\gamma_{2}}+T_{\bar{\nu} \nu_{1} \rightarrow \nu_{2}}^{\bar{\nu}} F_{\nu} F_{\nu_{1}}-T_{\nu \gamma_{2} \rightarrow \nu_{1}}^{\nu} F_{\nu} F_{\gamma_{2}}\right) . \\
& . \delta\left(k_{1}-k_{2}-k_{m}\right) \delta\left(\omega_{k_{1}}^{\nu}-\omega_{k_{2}}^{\nu_{2}}-\omega_{k_{m}}^{\nu}\right) d k_{1} d k_{m}
\end{aligned}
$$


- $27-$

$$
\begin{aligned}
& S_{\left(\nu_{2} \bar{\nu}_{1} \bar{\nu}\right)}^{\bar{\nu}}=\text { equation }(3.4 .8) \text { with indices } 1 \text { ind } 2 \text { interchanged, } \\
& \text { and } \\
& S_{\nu \nu_{1}}^{\nu}=-\int T_{\nu \nu_{1}}^{\nu} F_{\nu} F_{\nu_{1}} d k_{m}
\end{aligned}
$$

where $F_{\gamma}=F_{\gamma}\left(k_{i}\right), 3, P_{y_{j}}=F_{\gamma_{j}}\left(k_{j}\right)$, $\nu_{j}>0$ is any wave index, including $\nu$, ana

$$
T_{\nu_{1} \nu_{2} \rightarrow \nu}^{\nu}=72 \pi\left(\omega_{k_{2}}^{\nu}\right)^{2}\left|E_{-k_{1} k_{1} k_{2}}^{-\nu \gamma_{1} \nu_{2}}\right|^{2}
$$

$$
T_{\gamma \bar{\gamma}_{1} \rightarrow \nu_{2}}^{\nu}=72 \pi \omega_{k_{2}}^{\nu} \omega_{k_{2}}^{\nu_{2}}\left(R_{e}\right) \wedge(1,2)
$$

$$
T_{\gamma \bar{\gamma}_{2} \rightarrow \gamma_{1}}^{\nu}=72 \pi \omega_{k}^{\nu} \omega_{k_{1}}^{\gamma_{1}}\left(R_{e}\right) \Lambda(2,1)
$$

$$
\begin{aligned}
T_{\nu \nu_{1}}^{\gamma}= & -96 \omega_{k_{2}}^{\nu}\left(f_{m}\right) E_{-k_{n}-k_{1} k_{1}}^{-\nu_{\nu}-\nu_{1} \gamma_{1}}+144 \omega_{k}^{\nu}\left(P f_{m}\right) . \\
& \cdot \sum_{\gamma_{2}>0} \omega_{k_{2}}^{\gamma_{2}}\{\tilde{\Lambda}(1,2)+\tilde{\Lambda}(-1,2)-\tilde{\Lambda}(1,-2)-\tilde{\Lambda}(-1,-2)\}(3.4 .13)
\end{aligned}
$$

with

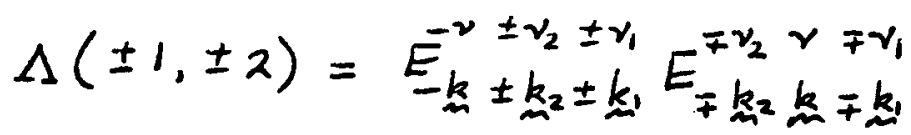




$$
\tilde{\Lambda}( \pm 1, \pm 2)=\frac{\Lambda( \pm 1, \pm 2)}{\left(-\omega_{k_{2}}^{\nu} \pm \omega_{k_{1}}^{\nu_{1}} \pm \omega_{k_{2}}^{\nu_{2}}\right)}
$$

The transfer functions of equation (3.4.8) follow from equations $(3.4 .10)-(3.4 .12)$ by changing the signs of the inaices $\nu_{j}$ and $k_{j}$ in the coupling coefficlents wherever components $\nu_{j}$ and anticomponents $\bar{\nu}_{j}$ are interchange ${ }^{2}$ in the transfer functions. Operators in parentheses apply to all later expressions in the transfer integrals. $P$ denotes the Chauchy principal value.

The interaction aiagrams associated with the various terms of the source function $\left.S_{\left(\gamma_{1}, \bar{\nu}\right)}^{\bar{\nu}}\right)$, equation (3.4.7), are shown in figure 2 .

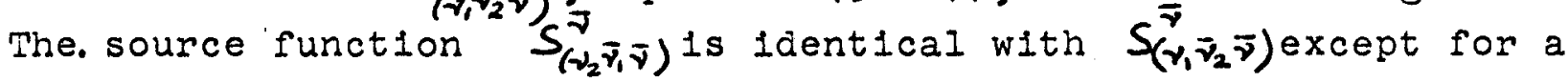
notational interchange of the inaices 1 and 2 . It has been listed as a alstinct source function, however, since the net energy transfer is obtalned by adaing all source function seperately.

In the case of conservative wave-wave interactions, the coupling coefficients $E$ are replaced by the symmetrical coefficlents $D$. The three transfer functions (3.4.10), (3.4.11) and $(3.4 .12)$ then become lientical except for a frequency factor, and the source functions $S_{\left(\nu_{1} \nu_{2}\right.}^{\bar{\nu}}, S_{\left(\nu_{1} \bar{\nu}_{2} \bar{\nu}\right)}^{\bar{\nu}}$ and $S_{\left(\nu_{2} \bar{\nu}, \bar{\nu}\right)}^{\bar{\nu}}$ can each be characterized by a single transfer function. The fourth source function $S_{\nu,}^{\gamma}$ vanishes, since the expressions in the parentheses $\{\ldots\}$ become real (section 3.6 ).

The interactions with external flelas ylelo the seven transfer expressions

$$
\begin{aligned}
S_{\left(\gamma_{1} \mu_{2} \bar{\nu}\right)=}^{\bar{\gamma}}= & \int\left(T_{\nu_{1} \mu_{2} \rightarrow \nu}^{\nu} F_{\nu_{1}} F_{\mu_{2}}-T_{\nu p_{2} \rightarrow \nu_{1}}^{\nu} F_{\gamma} F_{\mu_{2}}\right) . \\
& . \delta\left(k_{\mu_{1}}+k_{2}-k_{m}\right) \delta\left(\omega_{k_{1}}^{\nu}+\omega_{k_{2}}^{\mu_{2}}-\omega_{k_{2}}^{\nu}\right) d k_{1} d k_{m_{2}}
\end{aligned}
$$


$-29-$

$$
\begin{aligned}
& S_{\left(\nu_{1} \bar{\mu}_{2} \bar{\nu}\right)}^{\bar{\nu}}=\int\left(T_{\nu_{1} \bar{\mu}_{2} \rightarrow \nu}^{\nu} \bar{\nu}_{1} F_{\mu_{2}}-T_{\nu \bar{\mu}_{2} \rightarrow \nu_{1}}^{\nu} F_{\nu} F_{\mu_{2}}\right) . \\
& . \delta\left(k_{1}-k_{2}-k_{1}\right) \delta\left(\omega_{k_{1}}^{\nu_{1}}-\omega_{k_{2}}^{\mu_{2}}-\omega_{\underline{k}}^{\nu}\right) d k_{1} d \underline{k}_{2} \\
& S_{\left(\bar{\nu}_{1} \mu_{2} \bar{\nu}\right)}^{\bar{\nu}}=\int\left(T_{\bar{v}_{1} \mu_{2} \rightarrow \nu}^{\nu} F_{\nu_{1}} F_{\mu_{2}}+T_{\bar{\nu} \mu_{2} \rightarrow \nu_{1}}^{\bar{\nu}} F_{\nu} F_{\mu_{2}}\right) . \\
& \cdot \delta\left(k_{2}-k_{1}-k_{m}\right) \delta\left(\omega_{k_{2}}^{\mu_{2}}-\omega_{k_{1}}^{\nu_{1}}-\omega_{k_{2}}^{\nu}\right) d k_{1} d k_{2}
\end{aligned}
$$

$$
S_{\left(\mu_{1} \mu_{2} \bar{\nu}\right)}^{\bar{\nu}}=\int T_{\mu_{1} \mu_{2} \rightarrow \nu}^{\nu} F_{\mu_{1}} F_{\mu_{2}} \delta\left(k_{1}+k_{2}-k_{m}\right) \delta\left(\omega_{k_{1}}^{\mu_{1}}+\omega_{k_{2}}^{\mu_{2}}-\omega_{k}^{\nu}\right) d k_{1} d k_{2}(3.4 .19)
$$

$$
S_{\left(\mu_{1} \bar{\mu}_{2} \bar{\nu}\right)}^{\bar{\nu}}=\int T_{\mu_{1} \bar{\mu}_{2} \rightarrow \nu}^{\nu} F_{\mu_{1}} F_{\mu_{2}} \delta\left(k_{1}-k_{2}-k_{m}\right) \delta\left(\omega_{k_{1}}^{\mu_{1}}-\omega_{k_{2}}^{\mu_{2}}-\nu_{k}^{\nu}\right) d k_{2} d k_{2}(3.4 .20)
$$

$$
S_{\left(\mu_{2} \overline{\mu_{1}} \bar{\nu}\right)}^{\bar{\nu}}=\text { equation }(3.4 .20) \text { with indices } 1 \text { and } 2 \text { interchanged, }
$$

$$
\begin{aligned}
& S_{\nu \mu_{1}}^{\nu}=-\int T_{\nu \mu_{1}}^{\nu} F_{\mu_{1}}\left(k_{1}\right) E_{\nu}(k) d k_{\mu} \\
& \text { with } F_{\mu j}=F_{\mu_{j}}\left(k_{j}, \lambda, \lambda^{\prime}\right), \\
& \mu_{j}>0 \quad \text { and } \\
& T_{\gamma_{1} \nu_{2} \rightarrow \nu}^{\nu}=36\left(\omega_{k}^{\nu}\right)^{2}\left(R_{e}\right) E_{-k k_{\mu} k_{2}}^{-\nu \nu_{1} \nu_{2}}(\lambda)\left(E_{-k_{2} k_{1} k_{2}}^{-\nu \nu_{1} \nu_{2}}\left(\lambda^{\prime}\right)\right)^{*}
\end{aligned}
$$




$$
\begin{aligned}
& T_{\nu \bar{\mu}_{2} \rightarrow \nu_{1}}^{\nu}=36 \pi \omega_{k}^{\nu} \omega_{k_{1}}^{\nu_{1}}\left(R_{e}\right) \Lambda(2,1) \\
& (3.4 .23) \\
& T_{\mu_{1} \mu_{2} \rightarrow \nu}^{\nu}=72 \pi\left(\omega_{k}^{\nu}\right)^{2}(R e) \underbrace{-\nu \mu_{1} \mu_{2}}_{-k_{1} k_{1} k_{2}}\left(\lambda_{1}, \lambda_{2}\right)\left(E_{-k k_{1} k_{2}}^{-\nu \mu_{1} \mu_{2}}\left(\lambda_{1}^{\prime}, \lambda_{2}^{\prime}\right)\right)_{(3.4 .24)}^{*} \\
& T_{\nu \mu_{1}}^{\nu}=-32 \omega_{k}^{\nu}\left(F_{m}\right) E_{-k k_{n}-k_{1} k_{1}}^{-\nu \nu-\mu_{1}}+32 \omega_{k_{m}}^{\nu} . \\
& \cdot \sum_{\gamma_{2}>0} \omega_{k_{2}}^{\nu_{2}}\left(p y_{m}\right)\{\tilde{\Lambda}(1,2)+\tilde{\Lambda}(-1,2)-\tilde{\Lambda}(1,-2)-\tilde{\Lambda}(-1,-2)\}
\end{aligned}
$$

where $\Lambda$ and $\tilde{\Lambda}$ are defined as in equations (3.4.14), (3.4.15), the inalces $\gamma_{j}$ being replacea where appropriate by $\mu_{j}$. The first coefficient $E$ in the expressions for $\Lambda, \tilde{\Lambda}$ aepenas on $\lambda_{j}$, the second on $\lambda_{j}^{\prime}$.

The transfer functions of equations $(3.4 .17),(3.4 .18)$ and $(3.4 .20)$ are obtainea from equations $(3.4 .20)-(3.4 .22)$ as before by changing the signs of indices.

The source functions $(3.4 .16)-(3.4 .20)$ are similar in structure to the source functions $(3.4 .7)$ and $(3.4 .8)$ for wave-wave interactions. They are assoclated with the interaction-aiagram comblnations shown in f1g. 2. The source function $S_{\nu \mu,}^{\nu}$ is analogous to the function $S_{\nu \nu_{1}}^{\nu}$ and is characterized by the interaction alagram of fig. 3. (The numerical factors in the transfer functions aiffer from the corresponaing factors for wave-wave interactions. This is due to a difference in the admissable permutations of the inaices of the coupling coefficients. For external interactions, the permutations are restricted by the siae condition that wave inaices precede external inaices.) 
The total source function $S$ is given by the sum over all inaiviaual source functions, including all combinations of inaices $\nu_{j}>0, \mu_{j}>0$.

The derivation of closed transfer expressions in terms of the fiela spectra is based on certain statistical assumptions. In transfer expressions involving mixed spectral products of more than one field, the interacting fields are assumed to be statistically independent. In the case of interactions involving several components of the same fiela, the components are treated as statistically independent, $i . e$. the field is regaraed as Gaussian. The corresponaing transfer expressions are characterized by quadratic or higher-oraer proaucts of the spectrum of a single field. The linear transfer expressions involve no statistical assumptions.

The valiaity of these assumptions has been demonstrated for the case of weakly interacting wave fields by Prigogine (1962). The proof is rather complicatea, but can be unaerstood physically by interpreting the energy transfer in terms of interacting wave packets. The assumptions are then seen to be very similar to the Boltzmann hypothesis of statistical independence of interacting particles in a dilute gas (cf. Hasselmann, 1966). The physical argument can be similarly applied to support the hypothesis of statistical indepenaence between wave fielos and external fielas. On the other hand, the external fields themselves are in general neither statistically inaepenaent nor Gaussian. In this sense, the transfer expressions (3.4.19) and $(3.4 .20)$ are only approximate, the complete transfer expressions incluaing further integrals over cross spectra and thira and fourth cumulants. However, we shall not require these in our applications. The expressions $(3.4 .19)-(3.4 .20)$ can be a useful approximation in cases in which the cumulants are not well known, for example, in the problem of aerodynamically generated sound (cf. Lighthill, 1963). 


\subsection{Transfer diagrams}

The formal analysis of a weakly interacting system of wave fields ana external fielas has lead to thirteen distinct source functions at lowest order, most of which involve several transfer terms. . Thus even in rather simple systems, we may expect a wide variety of transfer processes (as we shall indeed find). To discuss these, some form of systematic nomenclature is clearly neeaed. It is again convenient to base this on a aiagram notation.

We shall refer in the present context to transfer aiagrams, as distinct from the interaction diagrams introduced in section 3.5 .

Each transfer term in a source function may be associatea with a particular component of a transfer aiagram. The superscript of the transfer functions in section 3.4 refers to the component, the group of subscripts to the diagram. We shall distinguish between two types of alagrams:

a) Scattering diagrams consist of a number of components or anti-components entering a vertex and a single wave component leaving the vertex. The frequencies and wavenumbers of the components satisfy the relations $(3.3 .1)$ and $(3.3 .2)$ for a resonant interaction aiagram.

b) Parametric diagrams consist only of a number of components entering a vertex. The diagram contains no anticomponents and no outgoing components. There is no restriction on the wave-numbers and frequencies.

We shall denote scattering diagrams by/symbols $\nu_{1} \nu_{2} \rightarrow \nu_{3} \nu_{1} \tilde{\mu}_{2} \rightarrow \nu_{2}$ etc., parametric diagrams by $\nu \nu_{1}, \nu \mu_{1}$, etc.

Scattering aiagrams/represent both conservative and non-conservative processes. There is always a net transfer of energy from the ingoing components to the outgoing component. In the case of conservative wave-wave interactions, the energy transfer of each component is proportional to the frequency of the component. The scattering diagrams can then be interpreted as collision alagrams in a particle picture (section 3.6 ). The wave-particle analogy is not applicable for non-conservative interactions. 
The aiagram resonance conaitions correspona to $\delta$-functions in the transfer integrals.

Parametric diagrams apply only to non-conservative interactions. They represent processes analogous to the parametric amplification of signals in non-linear electronic circuits. The rate of growth of a component is proportional to the power present in other components. In the simplest case, the diagrams contain only two components (excluding the degenerate linear case); there is no energy scattered into a third component. The diagrams are associated with transfer expressions which contain no

$\delta$-function resonance terms (the energy transfer is nontheless aue to resonant interaction aiagrams, cf. fig. 3). The aistinction between components and anti-comporents is therefore lost, as this is based - for transfer diagrams - on the sign combinations occuring in the resonance conditions.

The transfer expressions of any interacting system can be derived from the transfer diagrams with the aid of a single transfer rule: the rate of change of the spectrum of any wave component or antiwave component in a diagram is proportional to the product of the spectra of the ingoing components.

The lowest-oraer transfer expressions of section (4.4), for example, obtainea by applying the transfer rule to the components $\nu$ and $\bar{\nu}$ in the set of all transfer alagrams containing not more than two ingoing components. The transfer expressions $(3.4 .3),(3.4 .7)$, $(3.4 .8)$ and $(3.4 .16)-(3.4 .20)$ correspond to scattering aiagrams, the expressions $(3.4 .2),(3.4 .9)$ and $(3.4 .21)$ to parametric diagrams. Typical transfer diagrams are shown in figure 4. (The degenerate linear transfer expression (3.4.2) may be characterizea by either a scattering or parametric aiagram. In Hasselmann (1967 a) a scattering aiagram is used. A parametric diagram is in some respects preferable, cf. section 4.6). We coula aistinguish further between 'generating' processes, in which all ingoing components of a scattering alagram are external components, and'scattering processes proper; in which at least one ingoing component is a wave component. However, we shall not do this in the following. 
Scattering transfer expressions containing the same $\delta$-function resonance factors have been grouped into a single source function. The associated scattering alagrams define a diagram set. Members of a dlagram set are obtained by interchanging ingoing and outgoing components of a scattering alagram, the interchanged components changing s1gn (15 this loads to an outgoing ant1-wave component instead of a wave component, all components of the diagram change sign). For example, the diagrams $\nu_{1} \nu_{2} \rightarrow \gamma$, $\bar{\nu}_{1} \nu \rightarrow \nu$ and $\bar{\nu}_{2} \nu \rightarrow \nu_{1}$ represent a diagram set. We denote the set by the symmetrical symbol $\left(\nu_{1} \nu_{2} \bar{\nu}\right)$, , which lists all interacting components on the same side of the resonance equation. The source functions $\left.S_{\left(\nu_{1}\right.}^{\bar{\nu}} \nu_{2} \bar{\nu}\right)$ represents the net energj transfer of the wave component $\nu$ or $\bar{\nu}$ for all aiagrams of the $\operatorname{set}\left(\nu_{1} \nu_{2} \bar{\gamma}\right)$ (figure 4$)$.

We have introduced transfer äiagrams primarily às a notational convenience. They reflect the structure of the transfer expressions, but yield no information about the transfer functions themselves. These can be determined only from the detailea interaction analysis as characterized by the interaction diagrams. Comparison of figures 1-3 with figure 4 indicate that the interaction and tránsfer aiagrams of a given transfer expression are generally not very closely relatea.

However, in the case of conservative wave-wave interactions an interrelationship exists on account of the symmetry of the coupling coefficients. Both the Interaction analysis and the transfer expressions can be characterized in this case by a single set of aiagrams. The transfer rules become particularly simple if expressed in a particle plcture. They are closely related to the transition rules of quantum field scattering theory, and the diagrams themselves may be regarded as modified Feynman diagrams. 


\subsection{Conservative wave-wave interactions}

The lowest-order energy transfer due to conservative wave-wave interactions is given by

$$
\begin{aligned}
& S_{\left(\nu_{1} \nu_{2} \bar{\gamma}\right)}^{\bar{\gamma}}=\omega_{\underline{\underline{k}}}^{\nu} \int T_{\left(v_{1} \nu_{2} \bar{\gamma}\right)}\left(n_{1} n_{2}-n_{1} n-n_{2} n\right) \delta\left(k_{1}+k_{n}-k_{m}\right) . \quad(3.6,1) \\
& . \delta\left(\omega_{k_{1}}^{\nu_{1}}+\omega_{k_{2}}^{\nu_{2}}-\omega_{k_{2}}^{\nu}\right) d k_{1} d k_{2} \\
& S_{\left(\nu_{1} \bar{\nu}_{2} \bar{\gamma}\right)}^{\bar{\gamma}}=\omega_{k}^{\nu} \int T_{\left(\nu_{1} \bar{\nu}_{2} \bar{\nu}\right)}\left(n_{1} n_{2}+n_{1} n-n_{2} n\right) \delta\left(k_{1}-k_{2}-k_{n}\right) \cdot \quad(3.6 .2) \\
& \text {. } \delta\left(\omega_{\underline{k}_{1}}^{\nu_{1}}-\omega_{\underline{k}_{2}}^{\nu_{2}}-\omega_{k_{2}}^{\nu}\right) d k_{1} d k_{2}
\end{aligned}
$$

and

$S_{\left(\nu_{2}, \bar{v}, \bar{\gamma}\right)}^{\bar{\nu}}=$ equation $(3.6 .2)$ with inäices 1 and 2 interchanged, where $n=\frac{F_{\nu}(k)}{\omega_{k}^{r}}, n_{j}=\frac{F_{v_{j}}\left(k_{j j}\right)}{\omega_{k_{j}}^{v_{j}}}$

anò

$$
\begin{aligned}
& T_{\left(v_{1} v_{2} \bar{\gamma}\right)}=72 \pi \omega_{k_{1}}^{v_{1}} \omega_{k_{2}}^{v_{2}} \omega_{k}^{\nu}\left|D_{-k k_{1} k_{2}}^{-\nu v_{1} v_{2}}\right|^{2} \quad(3.6 .3)
\end{aligned}
$$

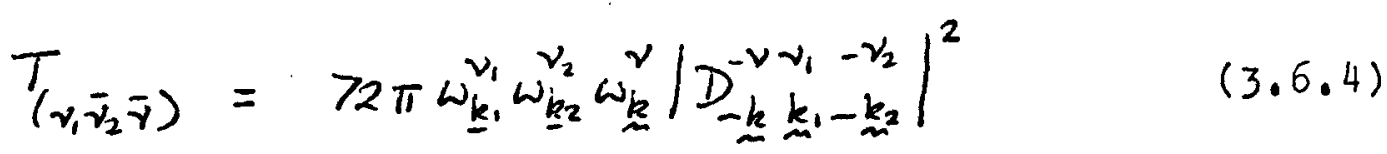

Equations $(3.6 .1)-(3.6 .4)$ follow from the general transfer expressions $(3.4 .7)-(3.4 .12)$ in the case of symmetrical coupling coefficients. They were first derived by Peierls (1929) for non-linear interactions between crystal lattice vibrations. 
We shall be interested primarily in wave-wave interactions within a gravity wave spectrum. On account of the negative curvature of the dispersion curve, it is not possible in this case to satisfy the resonance conditions with only three wave components (Phillips, 1960). The lowest order energy transfer Involves scattering from three components to a fourth,

where

$$
\begin{aligned}
S_{\left(g_{1} g_{2} g_{3} \bar{g}\right)}^{\bar{g}}=\omega_{k}^{g} \frac{D_{n}}{D t} & =\omega_{k}^{g} \int T_{\left(g_{1} g_{2} \bar{g}_{3} \bar{g}\right)}\left(n_{1} n_{2}+n_{3} n-n n_{1}-n n_{2}\right) \cdot(3 \cdot 6 \cdot 5) \\
& \cdot \delta\left(k_{1}+k_{2}-k_{m}-k\right) \delta\left(\omega_{k_{1}}^{g}+\omega_{k_{2}}^{g}-\omega_{k_{3}}^{g}-\omega_{m_{m}}^{g}\right) d k_{1} d k_{2} d k_{m}
\end{aligned}
$$

$$
\begin{aligned}
& T_{\left(g_{1} g_{2} \bar{g}_{3} \bar{g}\right)}=192 \pi \omega_{k_{1}}^{j} \omega_{k_{2}}^{g} \omega_{k_{3}}^{g} \omega_{k_{2}}^{g} \mid 2 D_{k_{1} k_{2}-k_{3}-k}^{g}-g-g
\end{aligned}
$$

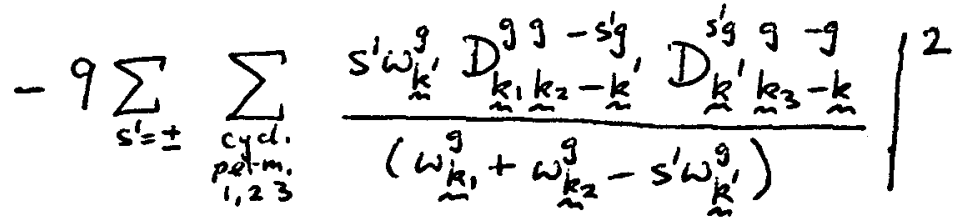

with $\omega_{k_{2}}^{g}, \omega_{k j}^{g}, \omega_{k^{\prime}}^{g}>0$ and $k_{m}^{\prime}=k_{1}+k_{m}$.

The coupling coefficients and transfer fuctions are given In Hasselmann $(1962,1963$ a). The general features of the computed transfer rates agree with observations mace by Snodgrass et al (1966), ef section ( 5.6 ).

The transfer expressions $(3.6 .1),(3.6 .2)$ and $(3.6 .5)$ have the general form of Boltzmann collision integrals for an ensemble of interacting particles, the spectra $n_{\nu}=F_{\nu} / w$ corresponaing to the number densities in $x-k$ phase space of particles of momentum $k$ ana energy $\omega$. The resonance conditions represent the conservation of energy and momentum, and the transfer functions correspona to interaction cross sections. 
The wave-particle analogy is unaerstanaable if one regards the interacting wave-system formally as the classical limit of a set of quantised fielas. The transfer expressions follow in this limit from the interaction rates of an ensemble of bosons (of. Peierls, 1,955).

An alternative particle picture which is not related to the rules of second quantisation may be defined in terms of an ensemble of both particles and anti-particles, anti-particles being characterized by negative energies, momenta and number densities. Although not realisable physically, the particle picture leads to simpler interaction rules and is more convenient for geophysical applications. The scattering diagrams may be interpreted in this picture as collision processes in which particles and anti-particles are created or annihilated.' The expressions for the transfer functions may be summarized by a few rules involving the coupling coefficients of interaction diagrams with the same inputs and output as the associated scattering diagram ( $f i g .5)$. In the case of the lowest-order processes, only one coupling coefficient occurs, and the expressions become particularly simple, equations (3.6.3), (3.6.4). We refer to Hasselmann (1966) for a summary of the interaction rules and their application to geophysical scattering problems. An acivantage of the particle analogy is that it determines the ratios of the energy and momentum transfer rates of all components of a scattering process. However, we shall be concerned here primarily with non-conservative processes, which can be characterized only by the general transfer rule stated in the previous section.

Once the general form of the transfer expressions has been establishea, the analysis of the wave energy balance of an interacting system is reduced to the determination of coupling coefficients: In the following, we consider the various coupling coefficients occuring in ocean wave interactions. 


\section{Interactions between gravity waves and the atmosphere}

\subsection{The lowest order processes}

We consiaer in this section the interactions between a gravitywave field ana a turbulent atmospheric boundary-layer. We shall assume that the boundary-layer flow consists of a mean horizontal velocity fiela $u_{m}=\left(U_{1}(z), U_{2}(z), 0\right)$ and a superimposed fluctuating field u $(x, z, t)$ which is statistically stationary and homogenous with respect to $x$.

The fluctuating fiela is characterizea by the spectrum

$$
F_{i j}\left(k, \omega, z, z^{\prime}\right)=\frac{u_{i, k, \omega}(z)\left[u_{j, k, \omega}\left(z^{\prime}\right)\right]^{*}}{\Delta k \Delta \omega}
$$

where $u_{i, k}, w$ is the Fourier component of the fluctuating velocity fiela, equation (3.1.13) (the index (a) refering to the atmosphere may be discarded in this section).

We shall find that the wave-atmosphere interactions can be expanaied in the form (3.1.14) with respect to the gravity-wave components $g$ and the turbulence Fourler components $t$; the mean flow enters only implicitly in the coupling coefficients. We are thus concerned formally with a two-component system.

The complete set of lowest-oraer transfer alagrams for this system are shown in figure 6. All combinations involving not more than three components occur, with the exception of the diagram set $\left(B_{1} g_{2} \bar{g}_{3}\right)$, which cannot satisfy the resonance conditions. Interactions between gravity waves and oceanic turbulence or currents are characterized by the same aiagrams, cf. section 5 .

Diagram (i) corresponds to Miles' linear interaction between the wave field and the mean boundary layer flow. A non-linear correction to Miles' theory is represented by alagram (11). 
The three diagrams (iii) correspond to the Eckart-Philiips theory of wave generation by random turbulent pressure fluctuations. They may be replaced more simply by the linear aiagram $p^{t} \rightarrow g$, where $p^{\text {t }}$ is the turbulent pressure at the surface. The remaining transfer diagrams (iv) and ( $v$ ) represent wave-turbulence interactions (Hasselmann, 1967 a). The net source function aue to these processes is given by the first four terms of equation $(2.2 .5)$.

4.2 The generation of waves by turbulent pressure fluctuations" Let

$$
\zeta(x, t)=\sum_{k} S_{k}(t) e^{i k \cdot x}
$$

and

$$
P(x, t)=\sum_{k} P_{k}(t) e^{i k \cdot x}
$$

be the Fourler representations of the surface elevation $\zeta$ and surface pressure $p$.

For an ideal fluid, the response of the wave components $S_{k}$ to the forcing pressure components $p_{k}$ is determined in the Iinear approximation by the harmonic oscillator equation

$$
\begin{aligned}
\ddot{S}_{k}+\sigma^{2} S_{k} & =-\frac{\sigma^{2}}{\rho g} P_{k} \\
& \left(\sigma \equiv \omega_{k}^{g}=(g k \tanh k H)^{1 / 2}\right) .
\end{aligned}
$$


Introducing stanaard wave variables, which in this case are given by

$$
\begin{aligned}
a_{k}^{g} & =\sqrt{\frac{\rho q}{2}}\left(\frac{\dot{s}_{k}}{\sigma}-i \zeta_{k}\right) \\
a_{k}^{-g} & =\sqrt{\frac{\rho g}{2}}\left(\frac{\dot{\zeta}_{k}}{\sigma}+i \zeta_{k}\right)
\end{aligned}
$$

equation $(4.2 .3)$ becomes

$$
\dot{a}_{k}^{g}+i \sigma a_{k}^{g}=-\frac{\sigma}{\sqrt{2 \rho g}} P_{k}
$$

For $p_{k}=0$, the free-wave solutions $a_{k}^{ \pm g}=\alpha_{k}^{ \pm g} e^{F i \sigma t}$ lead to thễ representation $(2.1 .1)$, with $\alpha_{k}^{g}=-i \sqrt{\frac{\rho g}{2} \eta_{k}}, \underline{\alpha}_{k}^{-g}=i \sqrt{\frac{\rho g}{2} \eta_{k}^{*}}$

The determination of the wave-atmosphere coupling coefficients reauces to the determination of the coupling field $p_{k}$ as a function of the interacting fielas. (We shall neglect the effect of surface shear stresses. In the linear approximation, shear stresses are not coupled to waves, but to rotational eajy motions and currents. We shall consiaer the interactions of waves with these motions in section 5 . The local transfer of wind energy to waves via shear stresses would require a three-fola coupling between waves, rotational flow in the ocean, ana air flow, which is probably negligible.)

To determine all the coupling cuefficients; we shall expana the surface pressure later in powers of the wave components and the turbulent velocity components. As first step, we consiaer here the interactions which involve turbulence components only. The lowest order energy transfer due to these processes is represented by the three diagrams of figure 6 (iii). However, since the pressure field in this case is simply the turbulent pressure $p^{t}$ in the absence of waves, it is more convenient to regard the surface pressure as the given external 
flelo instead of the velocity field. The transfer alagrams then reduce to the Iinear aiagram $\mathrm{p}^{t} \rightarrow \mathrm{g}$.

Introducing the Fourier representation

$$
p^{t}(x, t)=\sum_{k, \omega} p_{k, \omega}^{t} e^{i(k, x+\omega t)}
$$

and the three-aimensional pressure spectrum

$$
F_{p^{t}}(k, \omega)=\frac{P_{k, \omega}^{t}\left(P_{k, \omega}^{t}\right)^{*}}{\Delta k \Delta \omega}
$$

equations $(4.2 .5)$ and $(3.4 .3)$ yield the energy transfer

$$
\frac{D F(k)}{D t}=S_{(p \bar{g})}^{\bar{j}}=\frac{\pi \sigma^{2}}{2 g g} F_{p t}(k,-\sigma)
$$

We shall aiscuss the pressure spectrum in more detail in section $(4.6)$.

\subsection{The linear interaction with the mean boundary-layer flow} As next step in the expansion of the pressure field we consioer terms which are linear in the wave components but independent of the turbulence field. This requires investigating the velocity field $\delta y$ induced in the boundary layer by the waves. The problem has been consiciered in detail by Miles (1957,1959).

The velocity field $\delta$ may be representea as a superposition of two-dimensional flows characterized by stream-functions $\psi_{\mathrm{k}}$ and horizontal shear flows $\varphi_{\underline{N}}$,

$$
\delta u_{j}=\sum_{k}\left\{-\frac{k_{j}}{k} \frac{\partial \psi_{k}}{\partial z}+\eta_{j} \varphi_{k}\right\} e^{i k_{m} \cdot x}(j=1,2)(4.3 .1)
$$


$-42-$

$$
\delta u_{3}=\sum_{k} i k \psi_{k} e^{i k \cdot x}
$$

where $\mathcal{Z}$ is the horizontal unit vector perpendicular to $k$ and $\psi_{k}, \varphi_{k}$ are functions of $z$. The representation (4.3.1),

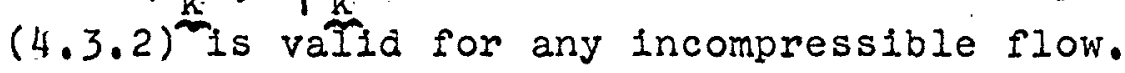

Since in the present approximation the flow is linear in the wave components and the interactions involve only the mean flow, we may write

$$
\psi_{k}=i(2 \rho g)^{-1 / 2}\left(w_{k_{m}}^{\sigma} a_{k}^{g}+w_{m}^{-\sigma} a_{k}^{-g}\right)
$$

$$
\varphi_{k}=i(2 \rho g)^{-1 / 2}\left(v_{k}^{\sigma} a_{k}^{g}+v_{k}^{-6} a_{k}^{-g}\right)
$$

where $w_{k}^{ \pm \omega}, v_{k}^{ \pm \omega}$ represent the response of the boundary layer to a periodic, unitamplituae surface displacement of (arbitrary) phase velocity $\pm \omega / k$. We need consider only positive frequencies, since $w_{k}^{\omega}=\left(w_{-k}^{-\omega}\right)^{*}, \varphi_{k}^{\omega}=\left(\varphi_{-k}^{-\omega}\right)^{*}$ on account of the reality of the fields.

Neglecting variations of the Reynolds'stresses, the perturbed equations of mean motion yield

$$
v_{k}^{\sigma}=-\frac{\frac{d \hat{U}}{d \underline{d}}}{\bar{u}-c} w_{w_{w}^{\sigma}}^{\sigma}
$$


$-43-$

ana the inviscid Orr-Sommerfeld equation

$$
L\left[w_{k}^{\sigma}\right]=\left\{(\bar{u}-c)\left(\frac{d^{2}}{d z^{2}}-k^{2}\right)-\frac{d^{2} \bar{u}}{d z^{2}}\right\} w_{k}^{\sigma}=0
$$

$(4.3 .6)$

where $\bar{u}=\frac{k \cdot u}{k}, \hat{u}=\eta \cdot u$ and $c=\sigma / k$

The neglect of the viscous terms in equations (4.3.5), $(4.3 .6)$ has been justified in greater detail by Benjamin (1959). \$T The appropriate boundary conditions are

$$
w_{k}^{\sigma}=(\bar{u}-c) \text { at } z=0
$$

and

$$
w_{k}^{\sigma}=0 \text { at } z=\infty
$$

Equation (4.3.7) follows from equations $(4.2 .4),(4.3 .2)$, $(4.3 .3)$ and the kinematic boundary condition

$$
\frac{D}{D t}(s-z)=\frac{\partial S}{\partial t}+u_{i} \frac{\partial s}{\partial x_{i}}-\delta u_{3} \quad \text { at } z=0
$$

where $\frac{D}{D E}$ denotes the substantial derivative. 
$-44-$

After solution of equations $(4.3 .6)-(4.3 .8)$, the pressure field at the surface follows from the horizontal equations of motion,

$$
p_{k}-p_{k}^{t}=\sum_{s= \pm} \frac{i \rho_{a}}{\sqrt{2 \rho g}}\left\{\left(\bar{u}_{0}-s c\right) \frac{d w_{k}^{s \sigma}(0)}{d z} a_{z}^{s j}-w_{k}^{s \sigma} a_{k}^{s \sigma} \frac{\partial \bar{u}_{0}}{d z}\right\} \quad(4.3 .9)
$$

where $\bar{U}_{0}=\bar{U}(0)$ and $\rho_{a}=$ density of air.

The linear coupling coefficient is obtained from equations $(3.1 .14)$ and $(4.2 .5)$,

$$
E_{-k k}^{-g g}=\frac{\sigma}{4 g} \frac{\rho a}{\rho}\left\{\left(\bar{u}_{0}-c\right) \frac{d w_{k}^{\sigma}}{d k z}-w_{k}^{\sigma} \frac{d \bar{u}_{0}}{d z}\right\} \quad \text { (4.3.10) }
$$

The energy transfer is then given by equation $(3.4 .2)$. .

The solution $w_{k}^{\sigma}$ of the Orr-Sommerfeld equation can normally be determined only numerically. However, the energy transfer can be expressed in an alternative form which is easier to estimate and illustrates more clearly the physical nature of the pressure feedback.

According to equation $(3.4 .4)$, the energy transfer depends only on the imaginary part of the coupling coefficient, $1 . e$, on the imaginary part of $\frac{d}{d z} w_{k}^{\sigma}(0)$. If we multiply equation $(4.3 .6)$ by $\left(w_{k}^{5}\right)^{*} /(\bar{u}-c)^{d z}$, subtract the complex conjugate expression and integrate from $z=0$ to $\hat{z}$, we obtain

$$
\eta_{m}\left[\frac{d w_{k}^{\sigma}}{d z}\left(w_{k}^{\sigma}\right)^{*}\right]_{0}^{\hat{z}}=\gamma_{m} \int_{0}^{\hat{z}} \frac{d^{2} \bar{u}}{d z^{2}} \frac{\left|w_{k}^{\sigma}\right|^{2}}{(\bar{u}-c)} d z
$$


Taking $\hat{z}=\infty$ and applying the boundary conaitions $(4.3 .7)$ and $(4.3 .8)$, this becomes

$$
Y_{m}\left(\frac{d w_{k}^{\sigma}(0)}{d z}\right)=\frac{1}{\bar{u}_{0}-c} \mathcal{J}_{m} \int_{0}^{\infty} \frac{d^{2} \bar{u}}{d z^{2}} \frac{\left|w_{k}^{\sigma}\right|^{2}}{(\bar{u}-c)} d z
$$

The integral is indeterminate. The singularity at the critical layer $\bar{U}-c=0$ arises from the singularity of the inviscid OrrSommerfela equation ana can be removed either by inclusion of the viscous terms or by treating the resonant response at the critical layer as a non-stationary inftial-value problem. The correct value of the integral is then found by indenting the integration path below the singularity (cf. Iin, 1955). Hence

$$
F_{m}\left(\frac{d w_{k}^{\sigma}(0)}{d z}\right)=\frac{\pi}{\bar{u}_{0}-c}\left(\frac{d^{2} \bar{u}_{c}}{d z^{2}} / \frac{d \bar{u}_{c}}{d z}\right)\left|w_{k}^{\sigma}\right|^{2}
$$

where the subscript c refers to values at the critical layer. The energy transfer follows then from equations $(3.4 .2),(4.3 .10)$ and $(4.3 .12)$,

$$
\frac{D F(k)}{D t}=S_{g}^{g}=-\frac{\pi \sigma^{2} \rho_{a}}{2 \rho g}\left(\frac{d^{2} \bar{u}_{c}}{d z^{2}} / \frac{d \bar{u}_{c}}{d z}\right)\left|\omega_{k}^{\sigma}\right|^{2} F(k)
$$

The essential feature of expression: $(4.3 .12)$ is the proportionality to the curvature-slope ratio of the velocity profile at the critical layer. The energy transfer is positive for normal profiles with negative curvature and positive wave slope. For a logarithmic profile, the energy transfer increases with decreasing height of the critical layer. Thus the Miles mechanism is particularly effective for waves with phase velocities appreciably lower than the wind velocity. For phase velocities greater than the maximal wind velocity or at angles greater than $90^{\circ}$ to the wind, the waves are neither damped nor enhanced. 
The energy and momentum is transfered to the waves entirely from the critical layer. This follows from equation (4.3.11) by noting that $\mathcal{I}_{m}\left[\frac{d w_{k}^{\sigma}}{d z}\left(w_{k}^{\sigma}\right)^{*}\right]=-2 \frac{\tau_{k}}{\rho_{a k}}$. where $\tau_{k}$ is the shear stress $\rho_{a} \overline{\delta u_{3} \delta u_{j}} \frac{k_{j}}{k_{k}}$ for a unit amplitude wave (the bar denotes the time mean). The shear stress is zero above the critlcal layer and constant between the critical layer and the surface. A physical explanation of en terms of the vortex forces acting on fluid particles near the critical layer has been given by IIghthIII (1962)。

We shall discuss measurements in connection with Miles' theory in section ( 4.7 ).

\subsection{Wave-turbulence interactions}

We consider now the terms in the pressure expansion which are Inear in the wave-field but contain arbitrary powers of the turbulence components. These arise from the wave-turbulence interactions in the equations for the wave-induced velocity field $\delta u_{0}$ We Iinearise as before with respect to $\delta u_{0}$. The viscous terms can again be neglected.

Including the cross interactions between the wave-induced field and the turbulence field, equations $(1.3 .5)$ and $(4.3 .6)$ become

$$
\begin{aligned}
& \tilde{L}\left[\psi_{k}\right]=\sum_{\substack{k^{\prime}+k_{i}^{\prime \prime}=k_{m} \\
\omega^{\prime \prime}, j}}\left\{A^{(1)} \psi_{k^{\prime}} u_{j} k_{2}^{\prime \prime} \omega^{\prime \prime}+A^{(2)} \varphi_{k^{\prime}} u_{j k^{\prime \prime}} \omega^{\prime \prime}\right\} e^{i \omega^{\prime \prime} t} \quad(i, 04.1)
\end{aligned}
$$

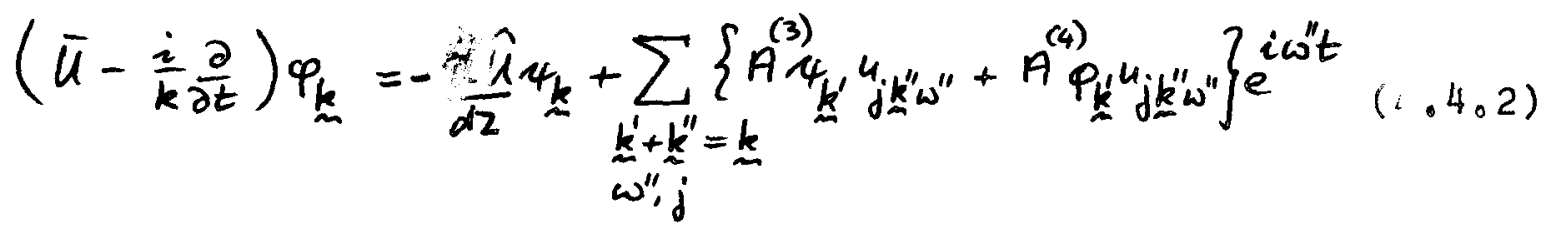


where

$$
\tilde{L}=\left(\bar{U}-\frac{i}{k} \frac{\partial}{\partial t}\right)\left(\frac{\partial^{2}}{\partial z^{2}}-k^{2}\right)-\frac{\partial^{2} \bar{u}}{\partial z^{2}}
$$

(we can now no longer apply the normalisation $(4.3 .3),\left(4.3 .4^{\prime}\right)$ ). The coupling coefficients $A^{(n)}=A^{(n)}\left(\underline{k} ! \underline{k}^{\prime \prime}, \omega^{\prime \prime}, j\right)$ are Iisted in the appendix.

The boundary conditions become

$$
\psi_{k}=\frac{i}{\sqrt{2 \rho g}} \sum_{s= \pm} s(\bar{u}-s c) a_{k}^{s g}+\sum_{\substack{k^{\prime}+k^{\prime \prime}=k \\ s_{1}^{\prime} \omega_{, j}^{\prime \prime} j}} B^{(1)} a_{k^{\prime} g} u_{j k^{\prime \prime} \omega^{\prime \prime}} e^{i \omega^{\prime \prime} t} \text { at } z=0(4.4 .4)
$$

and

$$
\psi_{k} \rightarrow 0 \text { for } z \rightarrow \infty
$$

where $B^{(1)}$ is given in the appenaix.

In the inviscic approximation, the bounaary conoltion (4.4.4) must be applied at the eage of the laminar sublayer. The turbulent velocity field at " $z=$ o" is horizontal, but non zero. Although the mean profiles and turbulent intensities vary rapialy in this region, the final results are insensitive to the precise definition of the sublayer thickness. They involve only the pressure fiela, which is effectively constant across the sublayer. (Similarly, in Miles' theory the boundary condition (4.3.7) is sensitive to the definition of $z=0$, but not the final transfer expression $(4 \cdot 3.12))$. 
The pressure at the surface is again given by the horizontal component of the equations of motion,

$$
\begin{aligned}
& p_{k}-p_{k}^{t}=\rho_{a}\left(\bar{u}-\frac{i}{k \partial} \frac{\partial}{\partial t}\right) \frac{\partial \psi_{k}}{\partial z}-\rho_{a} \psi_{k} \frac{\partial \bar{u}}{\partial z} \\
& +\sum_{k^{\prime} k^{\prime \prime}=k^{\prime}}\left\{c^{(1)} \psi_{k^{\prime}} u_{j k^{\prime \prime} \omega^{\prime \prime}}+c^{(2)} \varphi_{k^{\prime}} u_{j} k^{\prime \prime} \omega^{\prime \prime}\right\} e^{i \omega^{\prime \prime} t}
\end{aligned}
$$

The coupling ${ }^{j}$ coefficients $C^{(1)}$ and $C^{(2)}$ are listed in the appendix.

We attempt now to construct a solution to equations (4.4.1) $(4.4 .4)$ by expanaing $\psi_{k}$ and $\varphi_{k}$ in powers of the turbulence components,

$$
\begin{aligned}
& \psi_{k}=\psi_{k}^{(0)}+\psi_{k}^{(1)}+\psi_{k}^{(2)}+\cdots \cdot \\
& \varphi_{k}=\varphi_{k}^{(0)}+\varphi_{k}^{(1)}+\varphi_{k}^{(2)}+\cdots
\end{aligned}
$$

The leading term is the Miles' solution $\psi_{k}^{(0)}, \varphi_{k}^{(0)}$, which we assume to be a good first-order approximation. Observations by Ionguet-Higgins et al (1961) Indicate that this is indeed the case (cf. section 4.7 ).

We note that this aoes not necessarily imply that the energy transfer due to wave-turbulence interactions is small as compared with the Miles transfer. Miles' (1959) calculations inaicate that the surface pressure is almost $180^{\circ}$ out of phase with the surface elevation over the greater part of the wave spectrum (as one woula expect from a simple constant-velocity model). The energy transfer is due to the pressure component which is $90^{\circ}$. out of phase with the surface elevation, which is only a small fraction of the total pressure. Thus the Miles feed-back represents a small term in the flrst oraer theory; the higherorder pressure corrections can well be of the same order or larger. 
$-49-$

The nth order terms of the expansion are obtained by solving inhomogeneous Orr-Sommerfeld equations in which the $(n-1)-$ order terms appear as forcing terms in the bilinear expressions. Substituting the expansions in equations $(4.4 .1)-(4.4 .4)$ we obtain, using symbolic notation,

$$
\tilde{L}\left[\psi^{(n)}\right]=A^{(1)} \psi^{(n-1)} \ddot{m}+A^{(2)} \varphi^{(n-1)} \tilde{u}
$$

$(4.4 .7)$

$$
\left(\bar{u}-\frac{i}{k} \frac{\partial}{\partial t}\right) \varphi^{(n)}=-\frac{d \hat{u}}{d z} \psi^{(n)}+A^{(3)} \psi^{(n-1)} \ddot{u}+A^{(4)} \varphi^{(n-1)}{ }_{n}^{u}
$$

with boundary conditions

$$
\begin{aligned}
& \psi^{(1)}=B^{(1)} \psi^{(0)} a \\
& \psi^{(n)}=0, n \geqq 2 \quad \text { at } z=0 \\
& \psi^{(n)} \rightarrow 0 \quad \text { for } z \rightarrow \infty
\end{aligned}
$$$$
(4.4 .9)
$$

It can be shown that for the lowest-order transfer dependence of $a_{k}^{ \pm g}$ in equation (4.4.9) can be taken as the freewave time dependence $e^{\mp i \sigma t}$. The solutions can then be expressed in terms of the response functions $G_{k}^{w}\left(z, z^{\prime}\right)$ and $w_{k}^{w}(z)$, where

$$
\begin{aligned}
& L\left[G_{k}^{\omega}\left(z, z^{\prime}\right)\right]=\delta\left(z-z^{\prime}\right) \quad\left(z, z^{\prime}>0\right) \\
& G_{k}^{\omega}\left(0, z^{\prime}\right)=G_{k}^{\omega}\left(\infty, z^{\prime}\right)=0
\end{aligned}
$$


and $w_{\mathrm{k}}^{\omega}$ is defined by equations $(4.3 .6)-(4.3 .8)$,

$$
\begin{aligned}
& L\left[w_{k}^{\omega}\right]=0 \quad(z>0) \\
& w_{k}^{\omega}=\left(\bar{u}_{0}-\omega / k\right) \text { at } z=0 \\
& w_{k}^{w} \rightarrow 0 \text { for } z \rightarrow \infty
\end{aligned}
$$

The surface pressure is then obtained in the form

$$
p_{k}-p_{\underline{k}}^{t}=p_{\underline{k}}^{(0)}+p_{\underline{k}}^{(1)}+p_{\underline{k}}^{(2)}+\cdots \cdot
$$

where $\mathrm{P}_{\mathrm{K}}(0)$ is the Miles term and

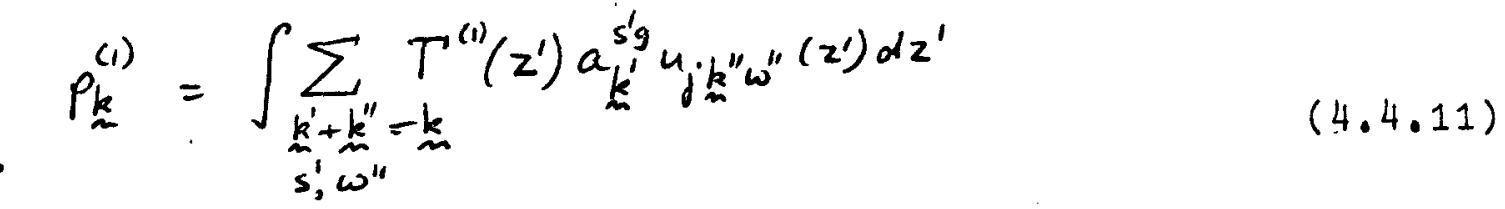

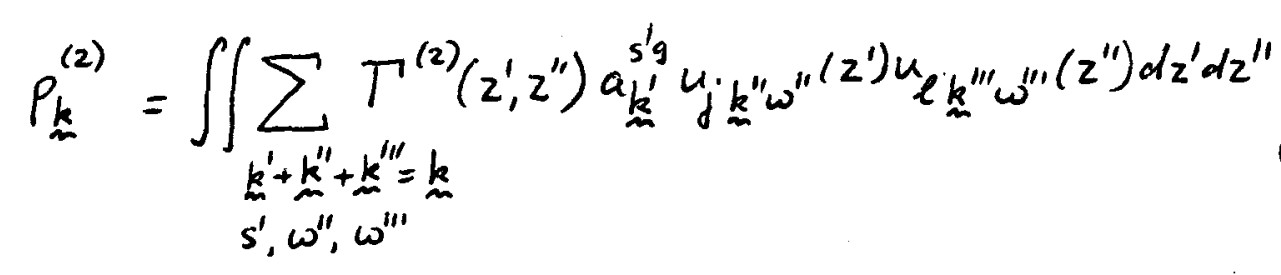

The coupling coefficients $\Gamma^{(1)}, \Gamma^{(2)}$ depend on the response functions and the coupling coefficients $A^{(1)}-A^{(4)}, B^{(1)}, C^{(1)}$ and $C^{(2)}$. They are given in the appenaix.

The coupling coefficients $E$ of equation (3.1.15) follow from equations $(4.2 .5),(4.4 .11)$ and $(4.4 .12)$. The energy transfer, finally, is ofiven by equations $(3.4 .14)-(3.4 .16)$ and $(3.4 .19)$. It may be written in the form 


$$
\begin{aligned}
& \frac{D F(k)}{D t}=\sum_{s= \pm} \int\left\{T_{i j}^{s}\left(\underset{m}{k}, k_{m}^{\prime}, z, z^{\prime}\right) F\left(k_{m}^{\prime}\right)-\hat{T}_{i j}^{s}\left(\underset{m}{k}, k_{m}^{\prime}, z, z^{\prime}\right) F(\underline{k})\right\} . \\
& \cdot F_{i j}\left(\underset{m}{k}+s k_{m}^{\prime}, \sigma+s \sigma^{\prime}, z, z^{\prime}\right) d k_{m}^{\prime} d z d z^{\prime} \\
&-F(\underset{m}{k}) \int \tilde{T}_{i j}\left(\underline{m}_{m}, k_{m}^{\prime}, \omega^{\prime}, z, z^{\prime}\right) F_{i j}\left(k_{m}^{\prime}, \omega^{\prime}, z, z^{\prime}\right) d k_{m}^{\prime} d \omega^{\prime} d z d z^{\prime}
\end{aligned}
$$

where the transfer functions $T_{i j}^{s}, \hat{T}_{i j}^{s}$ and $\widetilde{T}_{i j}^{s}$ are determined by equations $(3.4 .22),(3.4 .23)$ and $(3.4 .25)$.

The first integral of equation (4.4.13) represents the contributions from the three scattering diagrams, fig. 6 (iv). The secona integral corresponds to the parametric process, fig. $6(v)$. The scattering transfer expressions (3.4.16) and $(3.4 .17)$ have been reorderea with respect to components rather than diagrams. The first term of the first integral represents the net energy gained by the three outgoing components of the alagrams. The second term represents the energy lost by the three ingoing components. The three sign combinations have been reauced to two by using the symmetry relation

$$
F_{i j}\left(k, w, z, z^{\prime}\right)=F_{j i}\left(-k,-w, z^{\prime}, z\right)
$$

and Including both positive and negative frequencies in the transfer expression.

The first transfer function $T_{i j}^{S}$ is always positive; the remaining two may be of either sign.

The transfer functions depend on the response functions, which for an arbitrary profile can be determined only numerically. However, for idealised models such as a constant-velocity or line-segment profile, the functions become rather simple analytic expressions. In contrast to the laminar interaction problem, 
these models may yield acceptable approximations in the present case. In the laminar interaction problem, the constantvelocity model predicts the absolute value and phase of the surface pressure quite well for a falrly broad of range of phase velocities of the order of the wind velocity. However, since the energy transfer is zero to this approximation, the small phase shifts due to the critical layer are nevertheless lessentrat.

- In the case of wave-turbulence interactions, the phase shifts can be treated as higher-oraer effects, since an energy transfer occurs already in the first approximation of a constant-velocity profile.

\subsection{Non-linear wave-atmosphere interactions}

We consiaer finally the complete expansion of the surface pressure in which we include both the wave-turbulence interactions and the non-linear wave-wave-mean fow intections,

In symbolic notation, the complete equations of the waveinduced fielà are

$$
\begin{gathered}
\tilde{L}[\psi]=A^{(1)} \psi u \underline{m}+A^{(2)} \varphi \underline{\mu}+A^{(5)} \psi \psi+A^{(6)} \psi \varphi+A^{(7)} \varphi \varphi \\
\left(\bar{u}-\frac{i}{k} \frac{\partial}{\partial t}\right) \varphi=\frac{d \hat{u}}{d z} \psi+A^{(3)} \psi u+A^{(4)} \varphi \underline{m}+A^{(8)} \psi \psi+A^{(9)} \psi \varphi+A^{(10)} \varphi \varphi
\end{gathered}
$$

with boundary conaitions

$$
\begin{gathered}
4=\frac{i}{\sqrt{2 g g}} \sum_{s} s(\bar{u}-s c) a_{m}^{s g}+B^{(1)} a u+B_{m}^{(2)} a q+B_{a \varphi}^{(3)}+B_{a a}^{(4)}+\cdots \\
\text { at } z=0
\end{gathered}
$$

and

$$
u \rightarrow 0 \text { for } z \rightarrow \infty
$$


where $A^{(5)}-A^{(10)}$ and $B^{(2)}, B^{(3)}, \ldots$ are further coupling coeficients.

Similarly; the surface pressure is now

$$
\begin{aligned}
p-p^{t}= & \rho_{a}\left(\bar{u}-\frac{i}{k} \frac{\partial}{\partial t}\right) \frac{\partial \psi}{\partial z}-\rho_{a} \frac{d \bar{u}}{d z} \psi+c^{(1)} \psi u \\
& +c^{(2)} \varphi \varphi_{\mu} \\
& +c^{(3)} 44+c^{(4)} \psi \varphi+c^{(5)} \varphi \varphi+c^{(6)} 4 a+c^{(7)} \varphi a+\cdots
\end{aligned}
$$

with aditional coupling coefficients $c^{(3)}, c^{(4)}, \ldots$

The solutions can be constructed as before by expanding about the Miles solution with respect to both wave components ana turbulence components. We shall not go into detalls.

To lowest order, the only transfer expression not already incluaed in the previous analysis is the parametric process, diagram 6 (11).

$$
\frac{D F(k)}{D t}=S_{g g^{\prime}}^{g}=-F(k) \int T_{g g^{\prime}}^{g}\left(k, k_{m}^{\prime}\right) F\left(k_{m}^{\prime}\right) d k_{m}^{\prime} \quad(4.5 .6)
$$

The process $\mathrm{gg}^{\prime}$ is probably less important than the waveturbulence interactions, for the coupling coefficients are similar in both cases, but the wave-inauced velocity fluct-: uations are normaliy weaker than the turbulent fluctuations.

\subsection{The pressure spectra}

The relationship between the various transfer processes becomes clearer physically if one considers the surface-pressure alstributions.

The turbulent surface pressure $p^{t}$ of the unmodified bounary layer is characterized by a three-aimensional spectrum $F_{p t}(k, \omega)$ (.,) (section 4.2). The power spectrum of the free-wave 
field $\zeta$, on the other hana, is a two-aimensional distribution.

$$
F_{\zeta}(k, \omega)=\frac{1}{\rho g}\{F(k) \delta(\omega+\sigma)+F(-k) \delta(\omega-\sigma)\}
$$

confined to the positive and negative sheets of the dispersion surface $\omega= \pm \sigma(k)$. Interactions between the wave fiela and turbulent bouncary layer leaa to mixea two- ana three-aimensional aistributions for both pressure and wave fielös. For example, the linear interactions between the wave field and the mean flow yielas a two-aimensional pressure distribution on the dispersion surface. Conversely, the turbulent pressure fluctuations generate a three-dimensional continuum of forced waves.

The energy transfered to the waves is equal to the work done by the pressure against the surface. This is proportional to the quadrature spectrum of the surface pressure and wave'height, which is zero everywhere except on the dispersion surface. Thus the energy transfer is due entirely to the pressure components in resonance with free waves, and we need consider only the pressure aistributions on the dispersion surface.

The three-dimensional pressure continuum yieläs an energy transfer proportional to the three-aimensional pressure spectrum at the resonance frequency (see, for example, equation $(4.2 .7$ )).

The two-aimensional pressure alstribution yielas an energy transfer proportional to the wave spectrum (see, for example, equation (4.3.13)).

Three-dimensional pressure spectra are associated with scattering processes, two-dimensional distributions with parametric processes.

The aistributions of the lowest-oraer transfer processes are indicated schematically in figure 7 . 
The general form of the turbulent-pressure distribution follows from Taylor's hypothesis, which states that the frequency ano wave-number spectra of a turbulent fiela are approximately related as though the turbulence were a "frozen" spacial pattern convected a ownstream with the mean velocity of the flow. In our case, this implies that the turbulent pressure spectrum is concentratea about the surface $\omega+k_{1} u_{m}=0$, where $u_{m}$ is a "mean" bounarary-layer velocity. Since the velocity profile is curved, the effective mean velocity depenas on the eady scale $2 \pi / k$. It is not precisely aefined. The indeterminacy is generally of the same order as the spread of the pressure distribution about the surface $\omega+k_{1} U_{m}=0$. For simplicity, $\mathrm{U}_{\mathrm{m}}$ has been taken as constant in figure 7 .

Atmospheric turbulence spectra are normally peakec at consiaerably lower frequencles then wave spectra. Hence, in the range of wind-wave frequencies the turbulent pressure spectrum decreases with increasing frequency along the surface $w+k_{1} u_{m}=0$.

An appreciable energy transfer occurs only where the surface $\omega+k_{1} u_{m}=0$ intercepts the dispersion surface $\omega+\sigma(k)=0$, i.e. along the resonance curve $\sigma=k u_{m} \cos \varphi_{r}$, where $\varphi_{r}$ is the angle between the airection of wave propagation anci the wind. The longest waves are generated in the wind direction with a phase velocity equal to the wind speed. Shorter waves are generated at the angles $\varphi_{r}$ for which. the phase velocity $c / c o \varphi_{r}$ in wina direction equals the wind speea. (An alternative explanation of the resonance angle $\varphi_{r}$ in terms of the auto-correlation time scales of the pressure fluctuation is given in Phillips (1957).)

Linear interactions with the mean flow yield a two-dimensional pressure aistribution on the dispersion surface. The component. in quaarature with the wave height is proportional to the curvature/slope ratio of the wind profile at the critical layer. If we iaentify $U_{m}$ with the "anemometer wina speea", the enerfy transfer per unit wave height is effectively zero to the left 
of the resonance curve for a logarithmic profile and increases monotonicaliy with frequency for $\sigma>k u_{m} \cos \varphi_{r}$.

Wave-turbulence interactions yield both two- and threedimensional pressure distributions.

The scattering processes $g^{\prime} t \rightarrow g, \bar{g} t \rightarrow g$ and $\bar{g} t \rightarrow g$ (diagrams (iv), fig.6) are characterized by three-dimensional pressure spectra. The pressure fluctuations arise from quadratic interactions between turbulence and wave-inaucea velocity fluctuations. Since the most energetic turbulence components are at low frequencies, the sum and difference frequencies of the resultant pressure components lie close to the frequencies of the wave-inducea components. The same holds for the wavenumbers. Hence the pressure aistribution is concentrated about the dispersion surface, the maximum lying close to the maximum of the wave spectrum. (We have ignored weighting effects due to the coupling coefficients. A more detailed analysis shows that these ao not affect the conclusion.)

The parametric process gt (diagram (v), fig. 6) corresponas to a two-aimensional pressure aistribution. The pressure field arises from a cubic interaction between a wave-inducea component and two turbulence components of opposite wave-number and frequency (cf. figure 3 ).

The parametric process gg' (aiagram (ii), fig. 6) is similarly associated with a two-dimensional pressure distribution. In this case, the turbulence components of the process gt are replacea by a conjugate pair of wave-inouced components.

Estimates of the transfer rates are difficilt tomiakelovithoutut acitualty computing the transfer integrals.

Transfer rates of parametric processes are proportional to the air-water density ratio $\rho a / \rho$, whereas scattering processes. yiela an energy transfer proportional to $(\rho a / \rho)^{2}$. This suggests 
that parametric processes generally dominate over scattering processes. However, it should be noted that parametric prociesses depena critically on phase relationships, which can reduce the energy transfer consiaerably.

Similarly, turbulent velocity fluctuations are generally greater than wave-inducea velocities, so that the turbulent processes $t t^{\prime} \rightarrow \mathrm{g}, \ldots$. may be expected to dominate over the waveturbulence scattering processes $g^{\prime} t \rightarrow g, \ldots$ However, this is offset by the more favorable spectral aistribution of the wave-turbulence pressure fluctuations.

A reliable evaluation of the various transfer processes requires numerical calculations of the transfer expressions for typical boundary-layer models and comparison witr. observed wave growth and bouncary layers. Some progress in this eirection has been made, but our picture is still far from complete

\subsection{Comparison with observations}

Our analysis of wave-atmosphere interactions was based on the assumption that the wave-inauced perturbations could be described to first order by the linear interactions with the mean air flow. The hypothesis is supported by simultaneous measurements of wave height and surface pressure made byilionguet-Higgins et al. (1961) with a buoy. High coherency (0.8) between the wave and pressure recoras impliea a two-dimensional pressure spectrum, and over $90 \%$ of the coherent pressure was $180^{\circ}$ out-of-phase with the wave height, as would be expected for a mean-flow interaction. The observea pressure spectra agreed well with theoretical calculations of the $180^{\circ}$-out-of-phase component for a logarithmic profile. The resolution was inadequate to aetermine the energy transfer due to the small quadrature component of the pressure or to estimate the spectral density of the residual turbulent pressure. 
Ware grawth chas been in easured by Sinyder and Cox (:1966). The aevelopment of a single spectral component was aeterminea by towing a fourbuoy array seewaras from a lee shore at the group velocity of the wave component. The energy of the component was obtained from the array recoras by appropriate directional ana frequency filterlng. Only the $17 \mathrm{~m}$ wave-length component was analysed; 29 runs were made under varying wina conàitions.

The first parts of the growth curves were fitted to a MilesPhillips source function $S=\alpha+\beta F$. Initially, the $\alpha$-term dominated, and the wave growth was linear. As $F$ increased, the secona term became more important and the waves grew exponentically The major part of the wave energy was generated in the exponential phase.

The emperical value of $\alpha$ was found to be reasonably consistent with Phillips' transfer expression, assuming that threedimensional pressure spectra measured by Priestley (1965) over lana were typical also of the ocean.

The $\beta$-term was found to be larger than preaicted by Miles by a factor of 6 to 8 (figure 8 ). The theoretical values were basea on a logarithmic wina profile. Although wina profiles were not measurea, the experiments were performed under neutral conditions, for which logarithmic profiles are typical.

Barnett (1966) has measurea wave growth at higher wina speeds ( 40 knots) using as wave sensor an air-borne radar altimeter. The method yields the wave growth over a broad frequency band, but the airectional resolution: was smaller than in snyder and Cox's experiment. Only one case was analysed. The growth curves were again fittea to a source function $S=\alpha+\beta F$. The values of $\alpha$ and $\beta$, although more scattered, were consistent with Snyder and Cox's results, provided the turbulent pressure spectrum was scaled as the sixth power of the wind (Snycier ana Cox assumea a more plausible fourth-power relationship). The exponential growth rate was again consicierably larger than preaictea by Miles. 
The experiments indicate that neither Miles' nor Phillips' theory are capable of explaining the major part of the observea wave growth. This suggests that the remaining lowest-" order processes, i.e. the wave turbulence interactions (or, conceivably, non-linear interactions with the mean flow) are the principal source of wave energy. However, the conclusion shoula be treatea with caution, since the interaction theory is limitea to expansible interactions. Large, local disturbances, such as flow separation at the wave crests, are excluded. (In the case of flow separation, however, one would expect Jeffreys' (1926) sheltering theory to apply, which is similarly unable to explain the observea wave growth, cf. figure 8). The mechanism of wave generation in the ocean is still an open question.

It is of interest that Miles' mechanism has been verified in the laboratory for sinusoidal water waves (Shemain and Hsi, 1966) and artifical waves simulated by a moving sinusoidal belt (Zagustin et al., 1966). The phase shift of the wave-inauced perturbations across the critical layer was particulary clear in the latter experiment.

The natural turbulence spectrum in laboratory experiments is normally of too high frequency to stuay wave-turbulence interactions. However, laboratory investigations of this interaction mechanism by low-frequency moaulation of the mean air flow are feasible and would be of interest. 


\section{Interactions within the ocean}

\subsection{The lowest-oräer processes}

An incompressible velocity fielo in an infinite, non-rotating ocean may be aecomposea into surface gravity waves $\mathfrak{G}$, a mean current $U$ and a residual turbulent field $t$. If the fluid is stably stratified, the turbulence field can be decomposed further into internal sravity-wave modes $i_{1}, i_{2}, \ldots$ and a horizontal turbulence fieli $\mathrm{h}$. The decomposition is meaningful if the cross interactions between $i_{n}$ and $h$ are small comparea with the linear restoring forces of the internal modes. This is normally the case for small wave-numbers.

We consider first the aecomposition $g$, $\underset{m}{U}$ and $t$. As in the case of wave-atmosphere interactions, the equations of motion of the wave fiela $\delta$ can be expanced in powers of the components $g$ and $t$, the mean flow entering only implicitly in the expressions for the coupling coefficients. Formally, we are concerned with a two-component system $\delta$, $t$, and the lowest-order transfer. processes are icientical with the diagrams of figure 6 .

Diagram (i) represents the energy transfer aue to linear interactions with mean currents. It vanishes in the weak-interaction approximation, $|\mathrm{U}| \ll c$.

Diagram (ii) represents the parametric energy transfer gg' due to non-linear interactions with mean currents. The corresponaing interaction aiagrams are shown in figure 3 (the coupling coefficients aepena on the current profile). For $|\dot{U}| \ll c$, the transfer is non zero only for $|\mathrm{k}| \approx|\mathrm{k}|$.

Diagram (iii) represents the generation of waves by oceanic turbulence, which is probably unimportant. 
Diagrams (iv) ana (v) correspond, respectively, to scattering and parametric dampins of. waves by turbulence. The transfer rate of the parametric process is proportional to the wave spectrum, in accoraance with the heuristic concept of a turbulent "ediy viscosity". The eddy viscosity can be expressea as a Inear functional of the turbulence spectrum.

If the turbulence field is aecomposed further into internal modes $i_{n}$ and a horizontal turbulence fiela $h$, adaitional scattering processes into internal modes occur. However, these are normally unimportant for the energy balance of surface waves. (The process $\mathrm{g} \overline{\mathrm{s}} \rightarrow \mathrm{i}_{\mathrm{n}}$ and several internal-wave scattering processes have been investigated by Kenyon, 1966).

Scattering by a rancom ocean bottom can also be included in the weak-interaction theory, but will not be treatea here (cf. Hasselmann, 1966).

\subsection{The interaction equations}

Consiaer the interactions between a mean current $U=\left(U_{1}(z)\right.$,

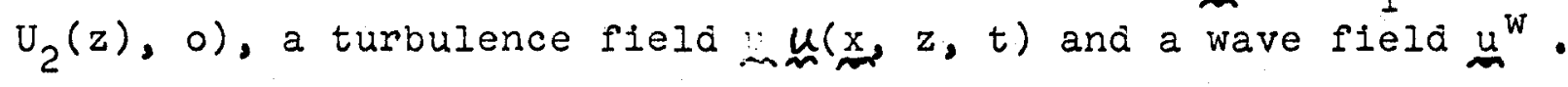

We assume that the turbulence and wave field are statistically homogeneous in $x$ ana that the density is constant through-out the fluid.

Let the superposition of the mean flow and the turbulence fiela represent a stationary turbulent shear flow which satisfies the equations of motion and the boundary condition at the bottom. At the surface, we assume $\zeta=0$, ana therefore $u_{3}=0$; the conaition of constant surface pressure is not satisfiea. (The fulfillment of both surface bounary conaitions for the complete flow is treatea as part of the wave-turbulence interaction.) We regarc the turbulent shear flow as given. 
We define the wave field as the potential flow

$$
u_{j}^{w}=\sum_{k} r_{k}(t) e^{i k_{m} \cdot x} \begin{cases}i k_{j} \frac{\cosh k(2+H)}{\cosh k H} & (j=1,2) \\ k \frac{\sinh k(2+H)}{\cosh k H} & (j=3)\end{cases}
$$

which satisfies the kinematic boundary conditions

$$
\begin{aligned}
\left(\frac{D}{D t}\right)(\zeta-z) \equiv \frac{\partial \zeta}{\partial t}+u_{1}^{w} \frac{\partial \zeta}{\partial x_{1}}+u_{2}^{w} \frac{\partial \zeta}{\partial x_{2}}-u_{3}^{w}=0 & \text { at } z=5
\end{aligned}
$$

and

$$
u_{3}=0 \quad \text { at } z=-H
$$

The wave field is uniquely determined by $\zeta(x, t)$ (except for a constant horizontal velocity, which we assume to be zero). In terms of the standard wave variables defined by equations $(4,2,4)$

$$
r_{k}=\frac{1}{\sigma} \sqrt{\frac{g}{2 g}}\left(a_{k}^{g}+a_{k}^{-g}\right)+\tilde{A}_{m}^{(1)} a+\tilde{A} a a a+\cdots \cdot
$$

where $A_{0}^{(1)} A^{(2)}$ oo are coupling coefficients determined by the non-linear terms in equation $(5,2,2)$ 。

The complete flow consists of the turbulent shear flow the

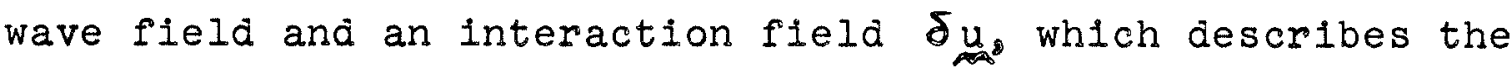
coupling between the wave field and the turbulence flow. 
The conations for weak coupling depend in detail on the type of interaction ana must be investigatea inaiviaually for. each transfer process. Generally, the turbulent shear flow can be treated as a perturbation of the wave fiela if both turbulent ana mean velocities are small compared with the wave phase velocities. Conversely, the wave fielo represents a perturbation of the turbulent flow if the energy transferea from the waves to the turbulent flow is small comparea with the total turbulent aissipation.

We describe the interaction field by the representation (4.3.1), (4.3.2). The equations for the components $\psi_{k}, \varphi_{\underline{k}}$ are obtained by subtracting the equations of motion of the turbulent shear flow from the equations of motion of the complete field,

$$
\begin{aligned}
\cdot \tilde{L}[\psi]= & \frac{d^{2} \bar{u}}{d z^{2}} \hat{\psi}+A^{(1)}(\psi+\hat{\psi}) \mu \\
& +A^{(2)} \varphi \underline{\mu} \\
& +A^{(5)}(\psi \psi+\psi \hat{\psi}+\hat{\psi} \psi)+A^{(6)}(\psi+\hat{\psi}) \varphi+A^{(2)} \varphi \varphi
\end{aligned}
$$

$$
\begin{aligned}
\left(\bar{u}-\frac{i}{k} \frac{\partial}{\partial t}\right) \varphi & =-\frac{d \hat{u}}{d z}(\psi+\hat{\psi})+A^{(3)}(\psi+\hat{\psi}) \underline{\mu}+A^{(4)} \varphi \underline{\mu} \\
& +A^{(8)}(\psi \psi+4 \hat{\psi}+\hat{\psi} \psi)+A^{(9)}(\psi+\hat{\psi}) \varphi+\dot{A}^{(10)} \varphi \varphi
\end{aligned}
$$

Equations (5.2.3), (5.2.4) are identical with equations (4.5.1), (4.5.2) except for adiitional terms involving the wavefield stream function

$$
\hat{\psi}_{k}=-i r_{k} \frac{\sinh k(z+H)}{\cosh k H}
$$


$-64 \cdot-$

The kinematic boundary condition at the surface,

$$
\frac{D}{D t}(\zeta-2)=0 \quad \text { at } z=\zeta
$$

yields, on account of $(5.2 .2)$,

$$
\begin{gathered}
\mu_{k}=\bar{U} S_{k}+\tilde{B}^{(1)} S_{u}+\tilde{B}^{(2)} \zeta(4+\hat{\psi})+\cdots \\
\text { at } z=0
\end{gathered}
$$

Similarly,

$$
4_{k}=0 \text { at } z=-H
$$

$(5.2 .8)$

The conation of constant surface pressure yields

$$
\begin{aligned}
\frac{\partial^{2}}{\partial z \partial t}\left(\psi_{k}+\hat{\psi}_{k}\right)-g i k \zeta_{k} & =-i k \bar{u} \frac{\partial}{\partial z}\left(\psi_{k}+\hat{\psi}_{k}\right)+i k \frac{d \bar{u}}{d z}\left(\psi k+\hat{\psi}_{k}\right) \\
& +\tilde{c}^{(1)}(\psi+\hat{\psi})_{u}+\tilde{c}^{(2)} \varphi \underline{\mu}+\cdots
\end{aligned}
$$

at $z=0$

Equation (5.2.9) follows by Taylor expansion of the condition

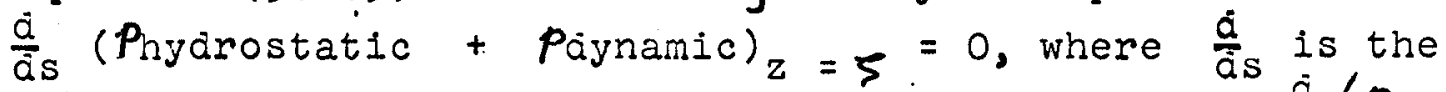
surface tangential derivative parallel to $\mathrm{k}$ and $\bar{a}$ (Paynamic) is expressed in terms of the velocities by means of the equations of motion.

Introducing standard wave variables in $\hat{\psi}_{k}$ and $\zeta_{k}$, and invoking equation (5.2.7), we obtain the wave equation

$$
\begin{aligned}
& \dot{a}_{k}^{g}+i \sigma a_{k}^{g}=\sigma \sqrt{\frac{\rho}{2 g}}\left(\bar{u}-\frac{i}{k} \frac{\partial}{\partial t}\right) \frac{\partial \psi k}{\partial z}+\frac{i}{2}\left(\frac{\sigma^{2}}{g k} \frac{d \bar{u}}{d z}-k \bar{u}\right)\left(a_{k}^{g}+a_{k}^{-g}\right) \\
& -\frac{i}{2} \frac{\sigma}{g} \bar{u} \frac{d \bar{u}}{d z}\left(a_{m}^{g}-a_{k}^{-g}\right)+\hat{c}_{m}^{(1)} \psi_{\mu}+\hat{c}^{(z)} \varphi_{m}+\cdots
\end{aligned}
$$


To determine the coupling coefficients, we neea to express the forcing terms on the right hand side of the equation in terms of the basic fielás $g, t$ ana $\underset{U}{U}$. This involves solving equations $(5.2 .4)-(5.2 .8)$ for the interaction fielás $\psi_{m}$ ana $\varphi_{k_{m}}$.

\subsection{Interactions with mean currents}

Interactions between waves and currents can be treatea as perturbations if $|\mathrm{d}| \ll c$, where $c$ is the phase velocity of the waves. In this case, the linear coupling coefficient $\mathrm{E}_{-k}^{-g} \mathrm{~g}$ is found to be real, and the energy transfer (3.4.2) vanishes. Linear interactions modify only the frequency ana velocity aistribution of the wave field. This is true generally for $\bar{U}<c$. As in Miles' problem, an energy transfer from the mean flow to the waves arises only through the phase shifts produced at a critical layer. In the present case, this would represent a strong interaction (since the coupling is not reduced by the factor $\rho a / s)$.

However, a weak energy transfer can occur at next oräer. Since secona-oraier scattering processes are excluaded by the negative curvature of the gravity-wave alspersion curve (section 3.6), we neea consicier only the parametric process $88^{\prime}$.

The relevant interaction diagram is shown in figure 9. The aifference interaction at the first vertex yielas a forced component $g^{\prime \prime}$ with a phase velocity $c^{\prime \prime}=\left(\omega-\omega^{i}\right) /\left|k k_{m}\right|^{\prime}$. If $c^{\prime \prime}>\bar{U}$, the coupling coefficients at both vertices are imaginary; the

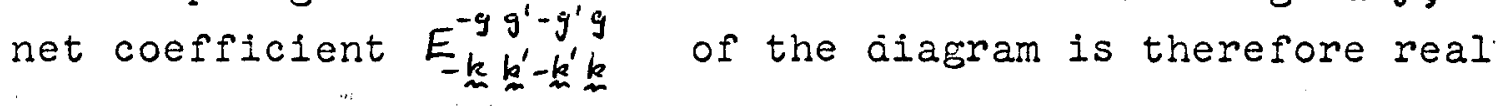
and yielas no contribution to the energy transfer $(3.4 .13)$. However, if $c^{\prime \prime}-\bar{U}$ passes through zero, a phase shift occurs in the first coupling coefficient, and an energy transfer results. 
For $\bar{U} \ll c$, a critical layer exists only for aifference interactions between components of approximately the same frequency. The net energy transfer is then of the form

$$
\frac{D F(k)}{D t} \equiv S_{g g^{\prime}}^{g}=-F(k) \int K\left(k, \varphi^{\prime}\right) F\left(k_{m}^{\prime}\right) d \varphi^{\prime} .
$$

where $k^{\prime}=k, \cos \varphi^{\prime}=\frac{k \cdot k^{\prime}}{k k^{\prime}}$

The kernel $K$ is a function of the mean current profile and can be expressea in terms of the response functions $w_{k} w_{k}$ and $G_{k}$ of section (4.4). The details of the analysis are similar to the case of wave-turbulence interactions and neea not be repeatea..

\subsection{Wave-turbulence interactions}

Interactions between waves and turbulence in the ocean yield the transfer processes shown in aiagrams (iv), ( $v$ ), figure 6 . The scattering processes (iv) involve interactions with turbulence scales of the same oraer or larger than a gravity wave length, whereas the parametric process ( $v$ ) depenas primarily on the small-scale turbulence structure.

\section{Wave-turbulence scattering}

The turbulence frequency $\omega_{t}$ can normally be neglected in the scattering conaition $\pm \omega^{\prime} \pm \omega_{t}=\omega$. It follows that a gravity-wave component $\tilde{b}^{\prime}$ of frequency $\omega^{\prime}$ is scatterea into a component $b$ of practically the same frequency but aifferent propagation airection. For a given component $\mathrm{g}^{\prime}$, the wave-number of the scattering turbulence component $k_{t}= \pm k^{\prime} \pm \frac{k}{m}$ is therefore confinea to the interval $0<k_{t}<2 k^{\prime}$; turbulence components of scale smaller than a half wave length ao not participate in scattering. 
In the approximation $\omega_{t} \ll \omega$, the energy of the gravity-wave field is conservec thy scattering processes (Hasselmann, 1966). The transfer expression follows from the form (3.6.1) for conservative wave-wave interactions in the limit of a zerofrequency fielä $\nu_{2}$,

$$
\frac{D F(\underline{k})}{D t} \equiv S_{\left(g^{\prime} t \bar{g}\right)}^{\bar{g}}+S_{\left(g^{\prime} \bar{g} \bar{g}\right)}^{\bar{g}}=\int K\left(k_{m}, k_{m}^{\prime}\right)\left\{F\left(k_{m}^{\prime}\right)-F(\underline{k})\right\} d \varphi^{\prime} \quad(5.4 .1)
$$

where $k^{\prime}=k, \cos \varphi^{\prime}=\frac{k \cdot k^{\prime}}{k k^{\prime}}$

(The source function $S_{\left(\bar{g}^{\prime} t \bar{g}\right)}^{\bar{g}}$, does not contribute, as the process $\overline{g^{\prime} t} \rightarrow \mathrm{g}$ is not compatible with the scattering condition for $\left.\omega_{t} \ll \omega, \omega^{\prime}\right)$. The kernel $K$ is symmetrical in $k$ and $k^{\prime}$ :and is a linear functional of the turbulence spectrum.

$$
\tilde{F}_{i j}\left(k_{n}^{\prime \prime}, z, z^{\prime}\right)=\int F_{i j}\left(k_{m}^{\prime \prime}, \omega^{\prime \prime}, z, z^{\prime}\right) d \omega^{\prime \prime}
$$

where $k_{n}^{\prime \prime}=\underline{m}-k_{n}^{\prime} \cdot F_{i j}$ is defined in equation (4.1.1).

The mean current $\mathrm{U}$ represents a small correction in the present problem and can be neglected. The response function of the interaction equations then reduce to exponentials and the transfer function occuring in $\mathrm{K}$ becomes a straightforward combination of the coupling coefficlents of equations (5.2.4) - (5.2.10). We shall not give $K$ explicitly, however, as it is alfficult to go further without more information about the turbulence spectrum $F_{1 j}$.

Phillips (1959) has estimated the decay of a single wave beam due to scattering, $1_{0}$ e. equation $(5.4 .1)$ with $F\left(\mathrm{k}^{\prime}\right)=0$, assuming an isotropic Kolmogoroff spectrum. The estimate is probably not very reliable, as the turbulence scales lie in the range in which the spectrum is strongly anisotropic, and the kernel $\mathrm{K}$ depends on both the scattering angle and the detalled tensor properties of $F_{i f}$. For the turbulence scales 
In question, it may be more appropriate to allow for the density stratification of the ocean and regard the threedimensional "turbulence" as a superposition of internal waves and a horizontal turbulence fleld. Formal expressions for scattering by internal waves and horizontal turbulence are given in Hasselmann (1966), but numerical estimates were not made. Kenyon (1966) has computed several cases of scattering between gravity-wave modes, but the processes considered were more relevant for the energy balance of internal waves than surface waves。

\section{Parametric damping ("eday viscosity")}

The parametric process $g$ t is determined by the interactions shown in figure 10. The contribution from a third diagram in which the components $g^{\circ}$ and $\xi^{\prime}$ of the second diagram are inter changed turns out o be negligible. A detailed analysis shows that the principal interactions involve small-scale turbulence components in the inertial subrange of the equilibrium spectrum.

The interactions can be determined, as before, by expanding the interaction fields in powers of wave components $a_{k}^{g}$ and turbulence components $u_{j k w}$. For the gt process we need retain only terms which are linear in $a_{k}^{b}$ and may therefore write

$$
\begin{aligned}
& 4_{k}=4_{k}^{(0)}+4_{k}^{(1)}+4_{k}^{(2)}+\cdots \\
& \varphi_{k}=\varphi_{k}^{(0)}+\varphi_{k}^{(1)}+\varphi_{k}^{(2)}+\cdots
\end{aligned}
$$

where the superscript refers to the power of the turbulence components.

For $\underline{U}=0$, the zero'th-order terms vanish, since the undisturbed wave solution is excluded in the definition of the interaction fiela. 
The first-order solution is determined by the equations

$$
\begin{array}{lll}
\tilde{L}\left[\psi^{(1)}\right]=A^{(1)} \hat{\psi} u & (5.4 .2) \\
-\frac{i}{k} \frac{\partial}{\partial t} \varphi^{(1)}=A^{(3)} \hat{\psi} \tilde{u} & (5.4 .3)
\end{array}
$$

with the bounary conaitions

$$
\begin{aligned}
& u^{(1)}=\tilde{B}^{(1)} S_{\tilde{s}} \text { at } z=0 \\
& (5.4 \cdot 4) \\
& \psi^{(1)}=0 \text { at } z=-H
\end{aligned}
$$

Higher-order solutions are determined by solving the equations $(4 \cdot 4 \cdot 7)-(4 \cdot 4 \cdot 10)$.

For $\underline{U}=0$, the inviscid Orr-Sommerfela operator reauces to the Laplacian form

$$
\tilde{L}=-\frac{i}{k} \frac{\partial}{\partial t}\left(\frac{\partial^{2}}{\partial z^{2}}-k^{2}\right)
$$

which enables the analysis tobe carried through explicitly. We neea not give the complete solution, however, since the major contribution arises from interactions with turbulence components of scale small comparea with a gravity wave length, 
which simplifies the analysis considerably.

In the firstoorder equations $(.4 .2)-(.4 .5)$, let $k$ ond $\mathrm{k}^{\prime \prime}$ be the respective wave numbers of the components $\hat{\psi}$ (wavefiela), $u$ (turbulence) and $\psi^{(1)}, \varphi^{(1)}$ (firstworaer interaction fiela) We assume that $k^{\prime} \ll k_{0}$ Then $k^{\text {pl }} \approx k^{p}$. since $k^{p *} k_{n}$ $+k_{0}$

The interaction field $\psi^{(1)}, \varphi^{(1)}$ may be represented as a superposition of two flelds $\psi_{v}^{(1)}, \varphi_{v}^{(1)}$ and $\psi_{s}^{(1)}, \varphi_{s}^{(1)}$, where $\psi_{v}^{(1)}, \varphi_{v}^{(1)}$ satisfies the inhomogeneous field equations $(.4 .2),(5.4 .3)$ with homogeneous boundary conditions and $\psi_{s}^{(1)}, \varphi_{s}^{(1)}$ is the solution of the homogeneous field equations with the inhomogeneous boundary conditions $(.04 .4)=(.04 .5)$.

Since the Green function $G\left(z, z^{\prime}\right)$ of the Laplace operator $(.4 .6)$ falls off as $e^{-k^{\prime \prime}\left|z-z^{\prime}\right|}$, the component $\psi_{v}^{(1)}(z)$ is determined, for large $k^{*}$, by the local values of the forcing function $A^{(1)} \hat{\psi} \mu$ in a thin layer of thickness $\delta=1 / k$. The component $\psi_{s}^{(1)}$ is sim.. ilarly limited to a surface layer of the thickness $\delta \cdot\left(\varphi_{v}^{(1)}\right.$ is rigorously local, and $\varphi_{s}^{(1)}$ vanishes).

The second-order fields $\psi^{(2)}, \varphi^{(2)}$ are determined by similar equations $(1.4 .7)-(1.4 .10)$ in which the products $\psi^{(1)} u, \varphi^{(1)} u$ occur as forcing terms. The wave-number of the u-component in this case is $-k^{p}$, so that the second-oraer excitation appears at the wave-number $k^{\prime \prime}-k^{\prime}=k$ of the gravity-wave component。 The net excltation involves a gravity-wave component and two turbulence components of opposite wave-number (figure 10)。

The forcing functions consist of surface terms and volume terms, both depending on small-scale turbulence components in a layer of thickness $\delta$. We assume that for these scales the turbulence can be regarded as locally isotropic. The integration over the layer can then be carried out and expressed in terms of the local scalar turbulence spectrum $E(k, \omega)$, where (cf。Batchelor, 1963) 


$$
F_{i j}(K, \omega)=\frac{\left\langle u_{i} \underline{\alpha} \omega\left(u_{j}, \omega\right)^{*}\right\rangle}{\Delta K \Delta \omega}=\frac{E(K, \omega)}{4 \pi}\left(\delta_{i j}-\frac{k_{i} K_{j}}{k^{2}}\right)_{(.0407)}
$$

and we have introduced the Fourier representation

$$
u_{j}(x, z, t)=\sum_{k, \omega} u_{j k} \omega e^{i\left(k_{m} x+k_{3} z+\omega t\right)}
$$

with respect to the three aimensional wave number $\mathrm{K}=\left(\mathrm{k}_{3} \mathrm{k}_{3}\right)$ 。 The wave equation $(.2010)$ involves the derivative $\frac{\partial u^{(2)}}{\partial z}$ at the surface. This is proportional to the surface forcing function plus an integral over the depth of the volume forcing function multiplied by $e^{k z}$ (for the present discussion, we take the depth as infinite, which eliminates the negative exponential $e^{-k z}$ in the Green function). Noting that the forcing functions are proportional to $\hat{\psi} \sim e^{k z}\left(a_{k}^{g}+a_{k}^{-g}\right)$, the wave equation finally reduces to the form

$$
\begin{aligned}
& \dot{a}_{k}^{g}+i \sigma a_{k}^{g}=-\gamma\left(a_{k}^{g}+a_{k}^{-g}\right) \\
& \gamma \equiv \gamma_{v}+\gamma_{s}=\int T(k, 4,8) \\
& \gamma, \omega) E(k, \omega) e^{2 k z} d k d \omega d z+\gamma_{s},(, 4.9)
\end{aligned}
$$

$\gamma_{s}$ depending on the turbulence spectrum at the surface. The wave aamping is given by the real part of $\gamma$.

$$
\frac{D}{D t} F(\underline{k}) \equiv S_{g t}^{g}=-2 \operatorname{Re}(\gamma) F(\underline{k})
$$


The coefficients $\gamma_{\mathcal{N}}$ and $\gamma_{s}$ involve interactions in surface layers of the thickness of a gravity wave length and a turbulence scale, respectively。 It may be expected that the energy transfer is not critically dependent on the turbulence characteristics in a thin surface layer of the order of a turbulence scale, and that therefore $\gamma_{s} \ll \gamma_{v}$ o However, an inspection of the interactions in terms of the velocity components indicates that this is not the case.

Let $\underset{m}{z}=u^{w}+\delta$ be the velocity of the combined wave and interaction fielas. Subtracting the equations of motion of the turbulent fiela $u$ from the equations of motion of the total fiela $u+v$, we obtain

$$
\frac{\partial v_{i}}{\partial t}=-\frac{1}{\rho} \frac{\partial \tilde{\omega}}{\partial x_{i}}-\frac{\partial}{\partial x_{j}}\left(v_{i} v_{j}\right)-\frac{\partial}{\partial x_{j}}\left(v_{i} u_{j}+v_{j} u_{i}\right)
$$

where $\widetilde{\omega}$ is the pressure difference between the fields $\underline{u}+\underline{v}$ and $u_{0}$ The viscous terms are found later to be negligible and have been discarded. Equation $(5.4 .11)$ is equivalent to equations $(0.2 .4),(0,2.5)$.

The boundary conditions are

$$
\begin{aligned}
& \frac{\partial S}{\partial t}-v_{3}+\sum_{j=1}^{2} \frac{\partial}{\partial x_{j}}\left(S u_{j}\right)=0 \text { at } z=0 \\
& \tilde{w}-g \zeta+5 \frac{\partial \tilde{\omega}}{\partial x_{3}}+5 \frac{\partial p}{\partial x_{3}}=0 \text { at } z=0
\end{aligned}
$$

and

$$
v_{3} \rightarrow 0 \text { for } z \rightarrow-\infty
$$


If the fleld $\mathrm{V}$ is expanded, as before, in powers of the turbulence components,

$$
\tilde{w}=v^{(0)}+w^{(1)}+w^{(2)}+\cdots
$$

we obtain for the second-order equation, following the analysis outined above,

$$
\frac{\partial v_{i}}{\partial t}=-\frac{1}{\rho} \frac{\partial \tilde{\omega}}{\partial x_{i}}+\frac{\partial}{\partial x_{j}} T_{\ddot{j}}^{(2)} \text { for } \quad z<0
$$

$$
\frac{\partial \zeta^{(2)}}{\partial t}-v_{3}^{(2)}=R^{(2)} \text { at } 2=0
$$

$$
\tilde{w}^{(2)}-g s^{(2)}=0 \text { at } z=0
$$

$$
v_{3}^{(2)} \rightarrow 0 \quad \text { for } 2 \rightarrow-\infty
$$

where the forcing terms $T_{i j}{ }^{(2)}$ and $R^{(2)}$ are proportional to the zero'th order wave-helght and the local turbulence spectrum.

The damping of the wave field can be deduced from energy considerations. If the energy spectrum

$$
\tilde{F}(k)=\frac{\rho}{2 \Delta k}\left\{g\left\langle S_{k} \zeta_{m}\right\rangle+\int_{-\infty}^{0}\left\langle v_{i k_{m}} v_{i-k}\right\rangle d z\right\}
$$


$-74-$

is expanded in powers of the turbulence components, $\tilde{F}=\tilde{F}^{(0)}+\tilde{F}^{(1)} \tilde{F}(r)$ one finds readily that to lowest order

$$
\frac{\partial \tilde{F}}{\partial t}=\frac{\partial \tilde{F}^{(2)}}{\partial t}=\frac{1}{\Delta k} \operatorname{Re}\left\{g \sum_{-k}^{(0)} R_{k}^{(2)}+\int_{-\infty}^{0} \frac{\partial T_{i j k}^{(2)} v_{i-k}^{(0)}}{\partial x_{j}} d z\right\}
$$

where the subscripts $k$ refer to Fourier components with respect to horizontal wave number, and

$$
\frac{\partial}{\partial x_{j}} \equiv \begin{cases}i k_{j} & (j=1,2) \\ \frac{\partial}{\partial z} & (j=3)\end{cases}
$$

The energy loss represents the work done against a surface The energy loss represents the work done against a surface
pressure proportional to $R_{k}^{(2)}$ and the volume stress-force $\frac{\partial T_{i j}^{(2)}}{\partial x_{j}}$
The essential feature of equation $(4.19)$ is that the work
per volume is associated with a stress force. The total energy
loss can therefore not be uniquely divided into volume and surface contributions. This may be seen by rewriting the stress term In equation (.4.19) as the difference between the work done by the surface s res and the dissipation $\Phi_{k}$.

$$
\operatorname{Re} \int_{-\infty}^{0} \frac{\partial T_{i j k}^{(2)} v_{i-k}^{(0)}}{\partial x_{j}} d z=\operatorname{Re}\left\{T_{i 3 k}^{(2)} v_{i-k}^{(0)}\right\}_{z=0}-\Phi_{k}
$$

where

$$
\Phi_{k}=\operatorname{Re} \int_{-\infty}^{0} T_{i j k}^{(2)} \frac{\partial v_{i-k}^{(0)}}{\partial x_{j}} d z
$$

The surface-stress term and the dissipation are comparable if the turbulence at the surface and within the fluid are of the same order 。

A quantitative estimate of the surface contributions is fficult, risc the turbulence in a space layer of the dimension of a turbulence scale is not isotropic. However, the dissipation $\Phi_{k}$ is insensitive to the turbulence properties in the thin surface layer and can be evaluated assuming local isotropy throughout. 
One finds, after some analysis (Hasselmann, 1967b)

$$
\begin{array}{ll}
\frac{D F}{D t}=-\beta_{\phi} F & (1.4 .20) \\
\beta_{\phi}=\frac{16 \pi k^{2}}{15} \int_{-\infty}^{0}\left\{\int_{0}^{\infty} E\left(K^{\prime}, \sigma\right) d K^{\prime}\right\} e^{2 k z} 2 k d z & .4021)
\end{array}
$$

Equations (4.20) - (4.21) are exact if the turbulerienoes not extend to the surface ( $e_{\circ} g$ o turbulence caused by breaking internal waves or internal shear layers).

In the inertial subrange, the scalar turbulence spectrum is given by (cf。Batchelor, 1963)

$$
E(k, \omega)=\varepsilon^{2 / 3} k^{-5 / 3} \omega_{0}^{-1} f\left(\omega / \omega_{0}\right)
$$

where $\rho \mathcal{E}$ is the turbulent energy dissipation per unit volume,

$$
\omega_{0}=\varepsilon^{1 / 3} K^{3 / 3}
$$

and $f(x)$ is a universal function. Turbulence measurements in a tidal channel by Grant et al。 (1962) indicate that $\int_{-\infty}^{+\infty} f(x) d x \approx 1.5$.

If the wave damping is due primarily to interactions in the inertial subrange, equation ( 5.4 .21 ) becomes

$$
\beta_{\phi}=\frac{\bar{\varepsilon} k \delta}{g}
$$


where $\bar{\varepsilon}=\int_{-\infty}^{0} \varepsilon(z) 2 k e^{2 k z} d z \quad$ is the weightea mean aissipation over the depth and $\delta=\frac{8 \pi}{5} \int_{0} x f(x) d x$ is a constant. (For finite depth, $\left.\bar{\varepsilon}=\int_{-H}^{0} \varepsilon \frac{\sinh ^{2} k(z+H)}{\sinh 2 k H} 4 k d z\right)$. Equation $(5,4.22)$ corresponds to a "turbulent viscosity" $\quad \nu_{t}=\frac{\bar{\varepsilon} \delta}{4 g^{k}}$

It is readily verified that the contributions to $\beta_{\phi}$ from interactions outside the inertial subrange are negligible。

At low we-numbers, $E\left(K^{\prime},-0, \rightarrow\right.$ since $\sigma / \omega_{0} \rightarrow \infty:$ the wave frequency becomes large compared with the turbulence frequencies as the turbulence scales approach a wave-lergth. The contribution from anisotropic turbulence of scales comparable with are larger than a wave-length is negligible provided the integral $\int_{0}^{\infty} x f(x) d x$ converges at infinity。 (This is ensured if the acceleration spectrum exists)。

At high wave numbers, the contribution from the dissipation range of the turbulence spectrum is negligible if $\sigma \ll \omega_{0}\left(K_{3}\right)=$ $\varepsilon^{1 / 3} K_{s}^{2 / 3}$. where $K_{s}=\varepsilon^{1 / 4} \nu^{-3 / 4}$ is the upper limit of the inertial subrange at which the viscous and inertial forces beocme comparable $\left(\nu=\right.$ viscosity). The conaition yielas $\varepsilon \gg \nu \sigma^{2}$ or, on account of equation $(5,4,22), \beta_{\phi} \gg \gamma k^{2} \delta$. The viscous decay factor for deepwwater waves is $\beta,=4 \nu k^{2}$ : the expression $(5.4 .22)$ is therefore valid provided the parametric damping is large compared with the laminar viscous damping. This is, in fact, the only case of interest, since the viscous damping is always negligible for ocean waves.

It remains to be verified that the interactions are weak from the point of view of the turbulence field. This is presumably the case if the energy gained by the turbulence through interactions with waves is small compared with the energy transfer due to internal turbulence interactions. The wave-turbulence energy transfer per unit volume at depth $z$ is $\frac{2 \varepsilon \delta}{9} \int F(k) k^{2} e^{2 k z} d z$ 
This is clearly small compared with $\varepsilon$ if the mean square wave slope is small, whlch we have assumed throughout。

Measurements of $\varepsilon$ in the open ocean have not been made, but lower bounds can be infered from the known tidal dissipation of the oceans. A uniform dissipation over all oceans corresponds to $\varepsilon \approx 10^{-5}$ ergs $\mathrm{cm}^{-3} \mathrm{sec}^{-1}$, which yields entirely negligible damping. However it is believed that the tidal energy is dissipated mainly in shallow seas of rather limited area. In these regions, the values of $\varepsilon$ may range from about 0.1 to maximally $10 \mathrm{ergsm}^{-3} \mathrm{sec}^{-1}$ (cf。Munk and MacDonald, 1960)。This corresponds to a damping factor for a $60 \mathrm{~m}$ wave of $\beta_{\phi}=10^{-7}$ to $10^{-5}$ secs, or a decay time of 100 days to 1 day. It appears that the parametric damping due to tidal turbulence is weak even in regions of high tidal dissipation. (We have assumed a fairly uniform distribution of turbulence. Localised wave-turbulence interactions in a boundary layer at the ocean bottom are more important, of。 section 5.5).

Another source of turbulence is white-capping。 In this case $\varepsilon$ is given by the energy lost by the waves through wave-breakingo Since we have seen that the energy loss due to wave-turbulence interactions is small compared with $\varepsilon$ if the mean-square wave slope is small, the parametric wave damping is negligible also in this case (in a fully-developed sea, the mean square wave slope is of the order of 0.02 )。

By the same reasoning, the "turbulent viscosity" will always be negligible unless a source of turbulence energy exists which is large compared with the energy lost by the waves。 It is difficult to find such a source in the open ocean. The energy transfer from the atmosphere is almost certainly too small. Excluding extreme situations such as very strong currents in shallow water, it appears that the turbulent viscosity. although often considered in wave-prediction methods, is not an important parameter in the energy balance of ocean waves. 


\subsection{Strong interactions}

Our applications of the general interaction formalism have been limited to interaction which are weak and expansible。 For ocean waves, all interactions are clearly weak in the mean, since the observed wave growth and decay times are large compared with a wave period. However, the interactions may be relatively strong in highly localized regions and can then no longer be expanded. Formally, the theory breaks down if abnormally large values of the skewness, kurtosis, etco associated with highly intermittent fields outweigh the expansion parameter in the moment expansions.

The transfer expressions can then no longer be truncated at the lowest-order moments (the spectra). As examples of such interactions we discuss briefly white capping and the damping of finite-depth waves by bottom friction.

\section{White capping}

Quantitative measurements and an adequate theory of white capping are both lacking. but it is generally believed that white capping is the principal dissipative mechanism balancing the generating processes in a "fully developed" equilibrium spectrum。

Phillips (1958) has suggested that for dimensional reasons white capping leads to an equilibrium frequency spectrum propor tional to $\omega^{-5}$. The power law has been confirmed by several measurements, the observed exponent varying between -4.5 and -5.5 . However, the dimensional argument is aifficult to support.

An $\omega^{-5}$ frequency spectrum corresponds to a two-aimensional wave-number spectrum

$$
F(k)=\operatorname{cgk}^{-4} s(\varphi, k)
$$


where $\varphi$ is the propagation direction, $s(\varphi, k)$ is the "spreading factor", normalized such that ${ }_{-\pi} \int_{\mathrm{s}}^{+\pi}(\varphi, k) d \varphi=1$, and $C$ is a constant. Phillips deduced the spectrum $(5.5 .1)$ essentially by assuming that the equilibrium spectrum was determined entirely by the white capping process and that this could be completely characterized locally by the three parameters g, $k$ and $F(k)$. However, this yielas an isotropic spectrum, since the parameters do not define a reference direction. The observed spectrum is a strongly anisotropic distribution $\left(s(\varphi) \sim \cos ^{4} \varphi\right)$ with the mean propagation parallel to the wind. This implies that the wind velocity is also an essential parameter of the problem, and the aimensional argument leading to the $\omega^{-5}$ law is not applicable。 ${ }^{+}$

It is, indeed, difficult to imagine an equilibrium spectrum which is independent of the energy input, unless the dissipative mechanism is envisaged as a strong, on off process which is effective only after the spectrum has exceeded a fixed, locally defined threshhold。 But it is improbable that white capping is local in $\mathrm{k}$-space. The visual impression suggests locality in $x$, and both properties are normally mutually exclusive. This is also indicated by the form of the spectrum (5.5.1). The instability conditions for white-capping are not known precisely, but it is generally believed that instability occurs when the local downward acceleration of the surface exceeds the gravitational acceleration $g$. Thus the root mean square acceleration of the surface is presumably one of the principal parameters characterizing white capping。 For equation (5.5.1), this quantity diverges at both ends of the spectrum. Thus the probability of white capping is determined not only by the equilibrium range, but depends also on the cut-off frequencies of the range.

F) Phillips actually applied the aimensional argument only to the one-almensional spectrum, which does not lead to a direct contradiction. However, in this case it must be assumed that the dependence on the wind velocity disappears after averaging our all propagation airections, which is difficult to justify physically。 
The effect of white capping on the equilibrium spectrum is not yet understood; it appears that dimensional arguments are inadequate and that the wave-breaking process itself needs investigating。

\section{Bottom friction}

The damping of finite-depth waves by bottom friction involves strongly non-linear, localized interactions in a non-stationary turbulent boundary layer. A rigorous treatment appears at present impossible。 We present here an approximate analysis (Hasselmann and Collins, 1967b) based on the empirical friction law

$$
\tau=-\rho c_{p} \mu|\underline{\mu}|
$$


where $\tau$ is the shear stress at the wall, $\mathrm{u}_{\mathrm{m}}$ is the flow velocity at the edge of the boundary layer and $c_{f}$ is a "constant" friction coefficient. Equation $(r, 5.2)$ is known to be a fair approximation for a wide range of turbulent flows. It has been tested for perlodic waves by Savage (1953), Iwagaki etal。 (1965) and Jonsson (1965), among others, and has been used in semiempirical wave prediction methods by Putnam and Johnson (1949) and Bretschneider and Reid (1954). The friction coefficient is, in fact, a slowly varying function of the flow parameters, but for the present first-order approach we shall regard $c_{f}$ as a constant.

We assume that the flow consists of a wave field $u^{W}$ and a mean current $\underline{u}^{c}$. We ignore interactions between the wave field and the mean current except in the turbulent boundary layer at the bottom. The complete flow in the $w=\underline{w}^{\mathrm{W}}+\underline{u}^{c}+\underline{u}^{t}$, where $\underline{u}^{t}$ is the turbulent velocity field in the bottom boundary layer. We regard $\underline{u}^{t}$ as zero outside a thin boundary layer of thickness $\delta \ll H_{0}$ (The wave velocity field is defined as the potential flow, equation $(5.2 .1)$, associated with the surface displacement 5 . We define $\underline{u}^{c}$ arbitrarily as constant in the boundary layer. The turbulent velocity is then given as the difference between the complete flow $w$ and the velocity fleld $\underline{u}^{W}+\underline{u}^{c}$ )。

The surface displacement may be represented as

$$
\zeta=\sum_{k} Z_{k}
$$

where to first order $z_{\mathrm{k}}$ are statistically orthogonal, free-wave components,

$$
z_{\underline{m}}=\hat{z}_{\underline{m}} \cos \left(\underline{k}_{0} x_{\infty}-\sigma_{t}+\widehat{\lambda}_{\underline{\underline{k}}}\right)
$$

with

$$
\begin{aligned}
& \left\langle z_{k}\right\rangle=0, \\
& \left\langle z_{k_{m}} z_{k^{p}}\right\rangle=\frac{\left\langle\hat{z}_{k} \hat{Z}_{k^{\prime}}\right\rangle}{2}=\frac{2 \Delta k F(k)}{\rho g} \delta_{k k^{\prime}} \quad(5.504)
\end{aligned}
$$


The wave velocity field is accordingly

$$
\underline{u}^{\mathrm{w}}=\sum_{\mathrm{W}}^{\mathrm{W}}
$$

where the components $U_{k}$ depend Iinearly on $Z_{k^{0}}$ In particular,

$$
u_{m}=\frac{g k}{6 \cosh k H} Z_{m} \text { at } z=-H
$$

On account of the boundary-layer interactions, the amplitudes $\mathrm{Z}_{\mathrm{k}}$ and phases $A_{\mathrm{k}}$ are not exactly constant, but vary slowly with time. To first order, the damping of the amplitudes can be determinec from the free-wave field by dalculating the work cone by the bottom stress against the freewwave velocity.

If we multiply the equations of motion of the complete flow

$$
\rho \frac{\partial w_{i}}{\partial t}=-\frac{\partial p}{\partial x_{i}}-\rho \frac{\partial}{\partial x_{j}}\left(w_{i} w_{j}\right)+\nu \rho \nabla^{2} w_{i}
$$

by $U_{i k}$, take expectation values and then integrate over the depth from $-H$ to $\zeta$ we obtain, applying the usual boundary conditions and the orthogonality property $(y 05)$,

$$
2 \Delta k_{m} \frac{\partial F(k)}{\partial t}=\left\langle\tau_{i} u_{i k}\right\rangle_{z=-H}-\int_{-H}^{-H+\delta}\left\langle T_{i j} \frac{\partial u_{i k}}{\partial x_{j}}\right\rangle d z-\frac{\partial}{\partial t} \int_{-H}^{-H+\delta}\left\langle\left(u_{i}^{t}+u_{i}\right) u_{i k}\right\rangle d z
$$

where $\quad T_{i j}=-\rho \frac{\partial}{\partial x_{j}}\left(w_{i} w_{j}\right)+\rho \nu \nabla^{2} w_{i}$

is the total stress

tensor and $\tau_{i}=T_{3 i}$

For $\delta \ll H$, the last two terms can be neglected, so that

$$
2 \Delta k \frac{\partial F(k)}{\partial t}=\left\langle\tau_{i} u_{i k k}\right\rangle_{2=-H}
$$

(tue factor 2 arises from the normalisation of $F(k)$ in section $\left.(2.1), \int F(\underline{k}) d \underline{m}=\frac{E}{2}\right)$. 
$-82-$

In deriving $(5,5,6)$ we invoked only the orthogonality of the Fourier components. However, if the interactions are weak in the mean, we may now substitute the free -wave velocities in the expression on the right hand side of the equation of or $\delta \ll H$, we may further replace the velocity $u_{u}=u^{w}+u^{c}$ at the edge of the boundary layer by the velocity at the wall in equation $(5.5 .2)$ for $\underset{n}{r}$. In the following, all velocities refer to the values at the wall.

It is convenient to introduce, for fixed $\mathrm{k}$, the variables

$$
\underline{u}=\underline{u}=\underline{\underline{u}}
$$

which are orthogonal to $\mathrm{U}_{\mathrm{K}}$

$$
\begin{gathered}
\left\langle u_{i}^{\prime} \cdot u_{j k}\right\rangle=0 \\
(\text { equations }(05.3)-(5,505))
\end{gathered}
$$

Since $\underline{u}^{\prime}$ differs from $\underline{m}$ by an infinitesmal quantity, we may express $\tau$ in terms of $\underline{u}^{p}, \underline{w}_{m}$ and then expand with respect to $\underset{\mathrm{K}}{\mathrm{U}}:$

$$
\tau=-\rho c_{f} u^{\prime}\left(\underline{u}^{\prime}+u_{k}\right)\left(1+\frac{u_{n}^{\prime} \cdot u_{k}}{u^{\prime}}+\cdots\right)
$$

so that

$$
\left\langle\tau_{i k} u_{i k}\right\rangle=-\rho c_{f}\left\langle u^{\prime}\left(u_{i}^{\prime} u_{i k}+u_{i k} u_{i k}+\frac{u_{i}^{\prime} u_{j}^{\prime} u_{i k} u_{j k}}{u^{\prime}}+\ldots\right)\right\rangle(5.507)
$$

always,

Now, a linear field of dispersive free waves is Gaussian (except for a short transition period after an initial, nonGaussian state, cf。Hasselmann, 1967 a). Since ur and $\mathrm{u}_{\mathrm{k}}$ depend lInearly on the wave field, they are jointly Gaussian. Moreover, they are statistically orthogonal, and one component, $\mathrm{U}_{\mathrm{N}^{\prime}}$ has 
$-83-$

zero mean. It follows that $\mu^{\prime}$ and $U_{k}$ are, in fact, statistically independent. The mean product of equation (3.5.7) can therefore be divided into two factors,

$$
\left\langle\tau_{i} u_{i k}\right\rangle=-\rho^{c} f\left\langle u_{i k} u_{i k}\right\rangle\left\langle u^{\prime}\right\rangle+\left\langle u_{i k} u_{j k}\right\rangle\left\langle\frac{u_{i \cdot}^{\prime} u_{j}^{\prime}}{u^{\prime}}\right\rangle
$$

We may now replace aug $^{\prime}$ again by $\mathrm{u}_{\mathrm{m}}$ and obtain, substituting in equation $(5.5 .6)$ and allowing for equations $(5.5 .4),(5.5 .5$.$) ,$

$$
\frac{\partial F(k)}{\partial t}=-\nu_{i j} k_{i} k_{j} F(k)
$$

where the anisotropic viscosity tensor

$$
\nu_{i j}=\frac{g c_{f}}{\sigma^{2} \cosh ^{2} k H}\left\{\delta_{i j}\langle u\rangle+\left\langle\frac{u_{i} u_{j}}{u}\right\rangle\right\}
$$

The quantities $\langle u\rangle,\left\langle\frac{u_{i} u_{j}}{u}\right\rangle$ are determined by the Gaussian probability distribution of the variables $u_{i}$. The mean of the distribution is equal to $\mathrm{u}^{c}$; the covariance matrix is determined by the spectrum,

$$
\mu_{i j}=\left\langle\left(u_{i}-u_{i}^{c}\right)\left(u_{j}-u_{j}^{c}\right)\right\rangle=\frac{2 g}{\rho} \int \frac{k_{i} k_{j}}{\sigma^{2} \cosh ^{2} k H} F(k) d k
$$

In the zero-current case, the men quantities of equation $(5,5,9)$ can be expressed in terms of complete elliptic integrals,

$$
\begin{aligned}
\langle u\rangle & =\sqrt{\frac{2 \mu_{\theta 1}}{\pi}} \underline{E} \\
\left\langle\frac{u_{1}^{2}}{u}\right\rangle & =\sqrt{\frac{2 \mu_{11}}{\pi}}\left(\frac{\underline{E}}{x^{2}}-\frac{\underline{k}}{x^{2}}\left(1-x^{2}\right)\right) \\
\left\langle\frac{u_{2}^{2}}{u}\right\rangle & =\sqrt{\frac{2 \mu_{11}}{\pi}}\left(\frac{1-x^{2}}{x^{2}}\right)(\underline{K}-\underline{E})
\end{aligned}
$$


where $x=\sqrt{1-\frac{\mu_{22}}{\mu_{11}}} \quad \underline{K}(x)$ and $E(x)$ are complete elliptic integrals of the first and second kina, respectively, and the coorainate system has been chosen such that $\mu_{12}=0, \mu_{22}<\mu_{10}$ The damping is a maximum in the mean propagation direction.

Figures 11 and 12 show two examples of the spectral decay computed for the case of a zero mean current and a mean current of $0.7 \mathrm{~m} / \mathrm{sec}$ at $45^{\circ}$ to the initial mean wave direction. The (constant) water depth of $100 \mathrm{~m}$ is representative of the North Sea. The initial energy distribution corresponas to a $40-\mathrm{knot}$ Pierson-Moskowitz spectrum, equation $(23,3)$, with a $\cos ^{4} \varphi$ spreading factor. The friction factor $c_{f}=0.015$ was determined by comparison of theoretical predictions with wave observations made at two offoshore stations at Panama City. Florida (Hasselmann and Collins, 1967). The value is consistent with other experimental data for periodic waves (cfo Jonsson, 1965), but is probably too large for the mean-current case. However, the same value of $c_{f}$ was taken in both cases for the sake of comparison.

It appears that wave damping by bottom friction can be quite important in shallow seas and continental margins. का we mention that although "the computations were based on the rather crude friction law $(5.502)$, this was not essential for the analysis. The same method can be applied for a more sophisticated friction law。

\subsection{Comparison with observations}

The direct method of investigating interaction processes experimentally is by cross-spectral or cross-bispectral analysis of the interacting fields. Unfortunately, measurements of this type are difficult to make, and one is limited largely to indirect evidence from wave observations. However, bispectral analysis was used by Hasselmann et al。(1963) to measure second-order wave interactions. In this case, the measurements involved only the wave field. 
The propagation of ocean swell over long aistances may be expected to depend more strongly on interactions within the ocean than wave-air interactions, and should therefore shed some light on the processes discussed in this chapter. We may include here also the wave-wave interactions discussed in section 3.6 。

Snodgrass et al. (1966) have measured swell attenuation in the Pacific. Waves radiated from storms South of New Zealand were recorded at six stations spaced at approximately equal distances along a $12,000 \mathrm{~km}=110^{\circ}$ great circle between New Zealand and Alaska. Twelve major storms were identified during the ten-week experiment. Only low-frequency storm waves $(\sigma / 2 \pi<0.1$ cycles per second) could be jetected above the local-sea background. (An increase in wind speed adas additional low-frequency waves to the "fully developed" spectrum, but does not appreciable affect the spectrum at intermediate and high frequencies, cf. equation $(2,3,3)$ )。

A significant attenuation was observed only within the first two or three thousand kilometers from the storm center. Over the remaining distance, the waves propagated virtually undamped.

The computed attenuation due to wave-wave scattering was found to be negligible for frequencies below 0.1 cycles per second at distances greater than a few thousand kilometers from the storm center. We have seen that the parametric damping due to small-scale turbulence is also small. The observations show further that wave-turbulence scattering is unimportant in most of the ocean. Scattering should have been noticable, besides by wave attenuation, by the late arrival of scattered energy, which was not detected。

The station spacing was inadequate for accurate measurement of the wave attenuation in the near zone. However, the general features of the observed decay were consistent with the computed energy transfer due to wave-wave scattering. Figure 13 shows a typical example of the spectra observed close to the storm. 
The spectrum in the generating region was not measured, but could be estimated from the winds reported in the generating region means the Pierson Moskowitz formula. The spectra are corrected for aistortion due to dispersion. The lower panels show the computed energy transfer aue to wave-wave scattering。 The small positive energy transfer at the peak appears to be typical of a peak close to a low-frequency cut-off (Hasselmann, $1963 \mathrm{~b}$ )。 The energy lost at intermediate frequencles is transfered to higher frequencies, not shown in the figures.

The travel time for 80 millicycle-per-second waves from the storm to the first station, Cape Palliser, New Zealand, was approximately $10^{5} \operatorname{secs}(1 \mathrm{day}$ ); from Cape Palliser to the second station, Tutulla, the travel time vas approximately $2.10^{5}$ secs. Bearing in mind that the transier rates in each figure refer to the initial spectrum, the wave wave scattering accounts quite well for the observed changes.

Beyond Tutuila, the spectral densities were reduced so far by dispersion and scattering that the computed scattering became negligible (except for angular beam broadening, which has no direct effect on the one-dimensional frequency spectrum). The observed attenuation beyona Tutulla was also negligible。 Wave-breaking or scattering by turbulence can not be excluded in the near zone by the data. However, the order-of-magnitude agreement with the computed transfer rates due to wave wave scattering demonstrate that wave-wave scattering is at least an important term in the net energy balance of the wave field. 
6. Conclusions

We have discussed the coupling between ocean waves and random fields in the ocean and atmosphere from the viewpoint of a general interaction formalism. The theory is complete, in the sense that we have been able to treat all lowest-order processes. However, it applies only to interactions which are weak and expansible.

The validity of the expansion procedure must be investigated for each process. A rigorous convergence proof was not attempted, but it can generally be verified that the expansions are consistent in the sense that the expansion parameters are small and the lowest-oraer interacting flelds may be regarded as statistically independeru. The convergence question is not associated with finite-oraer interaction theory as such. It arises already in the linear represensation of 'free' wave flelds. The interaction analysis is neither more nor less rigorous than the linear theory (excluding, of course the problem of statistical closure). Interactions with atmospheric turbulence are an important case in which this was not demonstrated, although the assumptions appear plausible and are valid for sufficiently small wave heights. The question can be resolved by trial computations or measurements.

Wave breaking and turbulent bottom friction are examples of non-expansible process. However, in both cases the interactions are weak in the mean. This property proved sufficient to determine the damping due to a non-analytic turbulent bottom stress。

The analysis for expansible interactions is basically straight forward, but can become involved algebraically. Interaction diagrams are useful in describing the structure of the expansion independent / / / gebraic details。 The net energy transfer is the result of many interaction combinations, which may be classed into scattering and 
parametric processes. Transfer alagrams afforà a concise notation for aistinguishing between the various processes. Even where the transfer functions are not known in detall, the general structure of the transfer expressions summarized by the transfer diagrams can be helpful in understanaing the overall energy balance of the wave field.

Numerical estimates exists at present for only a few processes: the wave-generation processes of Phillips and Miles, wave-wave scattering, and parametric damping by turbulence. We have not attempted to estimate rather complicated transfer expressions by order-of-magnitude dimensional considerations. However, computations based on simplifled boundary-layer models should be feasible. A more fundamental problem is estimating the energy loss due to white-capping, at present the one basically undetermined process in the radiation balance equation.

Only very few measurements bearing on the wave energy balance have been made, but these have proved extremely fruitful. Further experiments of this kind would be very desirable, Laboratory (and, if possible, field) studies of the interaction mechanisms by cross correlation techniques would also be invaluable in assessing the relative significance of the varlous processes. 


\section{Acknowledgments}

This article is based largely on work done while the author was at the Institute of Geophysics and Planetary Physics at the University of California, San Diego. The author is grateful for many stimulating discussions during this time with Drs, Munk, Cox, Miles, Gilbert, Backus and many others. The work was supported in part by the National Science Foundation under grant GP 2414. The work reported in the second part of section 5.5 was supported by the National Engineering Science Company。 
$-90=$

Appendix Coupling coefficients

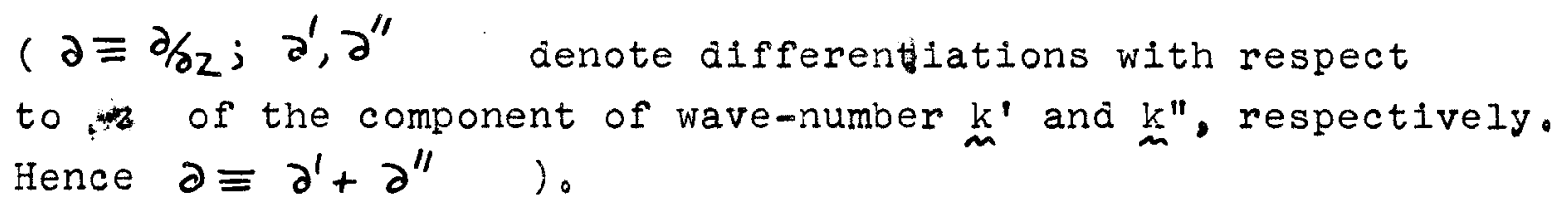

$$
A^{(\prime)}= \begin{cases}\frac{k^{\prime} k_{j}}{k^{2}} \partial \partial^{\prime \prime}-\frac{\left(k_{l}^{\prime} k_{l}^{\prime} k_{j}+k_{l}^{\prime} k_{l} k_{j}^{\prime}\right) \partial \partial^{\prime}}{k^{2} k^{\prime}}+k^{\prime} k_{j}^{\prime} & (j=1,2) \\ \frac{i k_{l}^{\prime} k_{l}}{k^{\prime} k^{2}} \partial \partial^{\prime} \partial^{\prime}+i \frac{k_{l}^{\prime} k_{l}^{\prime \prime}}{k^{\prime}} \partial^{\prime}-i k^{\prime} \partial & (j=3)\end{cases}
$$

$$
A^{(2)}= \begin{cases}\frac{\eta^{\prime} k_{l}^{\prime \prime}}{k^{2}}\left(k_{j}+k_{j}^{\prime}\right) \partial & (j=1,2) \\ -i \eta_{l}^{\prime} k_{l}^{\prime \prime}\left(\frac{\partial \partial^{\prime}}{k^{2}}+1\right) & (j=3)\end{cases}
$$

$A^{(3)}=\left\{\begin{array}{l}\frac{k_{l}^{\prime} k_{l}^{\prime \prime}}{k k^{\prime}} \eta_{j} \partial^{\prime}-\frac{k^{\prime} \eta_{j}}{k} \partial^{\prime \prime}+\frac{k_{l}^{\prime} \eta_{l} k_{j}^{\prime}}{k k^{\prime}} \partial^{\prime} \\ -i \frac{k_{l}^{\prime} \eta_{l}}{k k^{\prime}} \partial^{\prime} \partial^{\prime}\end{array}\right.$

$$
\begin{aligned}
& \left.C_{j}=1,2\right) \\
& (j=3)
\end{aligned}
$$

$$
A^{(4)}= \begin{cases}\frac{-\eta^{\prime} k^{\prime \prime} \eta_{j}}{k}-\frac{\eta^{\prime} \eta_{e} k_{j}^{\prime}}{k} & (j=1,2) \\ \frac{i \eta_{l}^{\prime} \eta_{l} \partial^{\prime}}{k} & (j=3)\end{cases}
$$


$-91-$

$$
\begin{aligned}
& B^{(1)}=\frac{1}{\sqrt{2 \rho g}}\left\{\begin{array}{l}
\frac{i s^{\prime} k_{j}^{\prime}}{2 k} \\
0
\end{array}\right. \\
& (j=1,2) \\
& (j=3) \\
& c^{(1)}= \begin{cases}\frac{\rho_{a}}{k^{2}}\left(k_{j} \frac{k_{e}^{\prime} k_{l}^{\prime \prime} \partial^{\prime}}{k^{\prime}}+\frac{k_{j}^{\prime} k_{l}^{\prime} k_{l}}{k^{\prime}} \partial^{\prime}-k^{\prime} k_{j} \partial^{\prime \prime}\right) & (j=1,2) \\
0 & (j=3)\end{cases} \\
& c^{(2)}= \begin{cases}-\frac{\rho_{a}}{k^{2}}\left(k_{j}^{\prime} \eta_{l}^{\prime} k_{l}+k_{l}^{\prime \prime} \eta_{l}^{\prime} k_{j}\right) & (j=1,2) \\
0 & (j=3)\end{cases} \\
& \left.T^{(1)}\left(z^{\prime}\right)=\frac{i s^{\prime}}{\sqrt{2 \rho g}} \frac{\partial}{\partial z}\left\{G_{\underline{k}}^{\omega}\left(z, z^{\prime}\right) w_{\underline{k}}^{s^{\prime} \sigma^{\prime}}\left(z^{\prime}\right)\right\}\right\}_{z=0}\left\{A^{(1)}-\frac{A^{(z)} \frac{d \hat{u}^{\prime}}{d z}}{\left(\overline{u^{\prime}}-s^{\prime} \sigma^{\prime} / k^{\prime}\right)}\right\} \\
& +\delta\left(z^{\prime}\right)\left\{\frac{i s^{\prime}}{\sqrt{2 \rho g}}\left[c^{(1)}\left(\overline{u^{\prime}}-\frac{s^{\prime} \sigma^{\prime}}{k^{\prime}}\right)-c^{(z)} \frac{d \hat{u}^{\prime}}{d z}\right]\right. \\
& \left.+\rho_{a} B^{(1)}\left[\frac{d w_{k}^{\omega}}{d z^{\prime}}-\frac{d \bar{u}}{d z^{\prime}}\right]\right\}
\end{aligned}
$$

where $\bar{U}^{\prime}=\frac{\underline{u} \cdot k^{\prime}}{k^{\prime}}, \hat{U} \cdot=\underline{U} \cdot \underline{y}^{\prime}$ and all terms are taken a ty $z^{\prime}$, unless otherwise indicated. The arguments of the coefficients $A^{(1)}, A^{(2)}, B^{(1)}, C^{(1)}$ and $C^{(2)}$ are the same as in the expressions given above. 
$-92-$

$$
\begin{aligned}
& T^{(z)}\left(z, z^{\prime}\right)=\left\{\frac{\partial}{\partial z} G_{k}^{\omega}\left(z, z^{\prime \prime}\right)\right\}_{z=0}\left\{\alpha_{j l} G_{k^{0}}^{\omega^{0}}\left(z^{\prime \prime}, z^{\prime}\right)+\beta_{j l} \delta\left(z^{\prime}\right) w_{k^{0}}^{\omega^{0}}\left(z^{\prime \prime}\right)\right. \\
& \left.+\gamma_{j l} \delta\left(z^{\prime}-z^{\prime \prime}\right) z_{k^{0}}^{\omega^{0}}\left(z^{\prime \prime}\right)+\varepsilon_{j l} \delta\left(z^{\prime}\right) \delta\left(z^{\prime \prime}\right)\right\}
\end{aligned}
$$

where

$$
\begin{aligned}
& \alpha_{j l}=\frac{i s^{\prime} \rho_{a}(\bar{u}-\omega / k)}{\sqrt{2 \rho g}}\left\{A_{l}^{(1)}-\lambda^{0} A_{l}^{(2)}\right\}\left\{A_{j}^{(1)}-\lambda^{\prime} A_{j}^{(2)}\right\} \\
& \beta_{j l}=\frac{\rho_{a}(\bar{u}-\omega / k)}{\left(\overline{u^{0}}-\omega^{0} / k^{0}\right)}\left\{A_{l}^{(1)}-\lambda^{0} A_{l}^{(2)}\right\} B_{j}^{(1)} \\
& \gamma_{j l}=\frac{i s^{\prime}}{\sqrt{2 \rho g}} \frac{\rho_{a}(\bar{u}-\omega / k)}{\left(\overline{u^{0}}-\omega^{0} / k^{0}\right)} A_{l}^{(2)}\left\{A_{j}^{(3)}-\lambda^{\prime} A_{j}^{(4)}\right\} \\
& \varepsilon_{j l}=\left\{C_{l}^{(1)}-\lambda^{0} C_{l}^{(2)}\right\} B_{j}^{(1)}+\frac{i s^{\prime}}{\sqrt{2 \rho g}} C_{l}^{(2)}\left\{A_{j}^{(3)}-\lambda^{\prime} A_{j}^{(4)}\right\} \\
& \text { with } k_{2}^{0}=k^{\prime}+k^{\prime \prime} \\
& \lambda^{0}=\frac{d \hat{u}^{0}}{d z} /\left(\overline{u^{0}}-\omega_{/ 0}^{0}\right) \\
& \lambda^{\prime}=\frac{d}{d z} \hat{u^{\prime}} /\left(\overline{u^{\prime}}-\omega^{\prime} / k^{\prime}\right)
\end{aligned}
$$

The arguments of the coefficients $A_{j}^{(1)}, A_{j}^{(2)}, \ldots$ are the same as in the previous expressions. The wave-numbers $k^{\prime}$ and $k^{\prime \prime}$ in the coefficients $A_{I}^{(1)}, A_{I}^{(2)}, \ldots$ are replaced by $\mathrm{K}_{\mathrm{K}}^{0}$ and $\mathrm{K}^{\prime \prime}$, respectively, ana $\mathrm{J}$ is replaced by 1 . 


\begin{tabular}{|c|c|c|}
\hline Backus, G.E. & $(1962)$ & Deep Sea Res. 9, 185-197. \\
\hline Baer, L. & $(1962)$ & $\begin{array}{l}\text { Lockhea Missile anà Space Co., } \\
\text { - Rep.-801296. }\end{array}$ \\
\hline Barnett, T.P. & $(1966)$ & Ph.D, thesis, Univ, Cal, , San Diego. \\
\hline Batchelor, G.K. & $(1963)$ & $\begin{array}{l}\text { The Theory of Homogeneous turbulence. } \\
\text { Camb. Univ. Press. }\end{array}$ \\
\hline Benjamin, B.T. & (1959) & Journ, Fluid Mech, 6, 161-205. \\
\hline Bretschneider, C.I. & (1959) & Beach Erosion Boara. Tech.Mem. No.118 \\
\hline $\begin{array}{l}\text { Bretschneider, C.I. } \\
\text { \& R.O. Reid }\end{array}$ & $(1954)$ & Beach Erosion Boara Tech.Mem. No. 45 . \\
\hline Burling, R. W. & $(1959)$ & Dtsch.Hyörogr.z. 12, 45-117. \\
\hline Chanörasekhar, $S$. & (1959) & Raciative Transfer, Dover Publ. \\
\hline Darbyshire, J. & $(1955)$ & Proc.Roy.Soc.A., 230, 560-569. \\
\hline Darbyshire, J. & (1959) & Dtsch.Hyärogr.z. 12, 1-13, 196-203. \\
\hline Dorrestein, $R$. & $(1960)$ & Journ.Geophys.Res. 65, 637-642. \\
\hline Eckart, $G$. & $(1953)$ & Journ. Appl. Phys. 24, 1485-1494. \\
\hline Fons, $\mathrm{C}$. & $(1966)$ & Cahiers Oceanogr. 18, 16-33. \\
\hline
\end{tabular}

Gelci, R, ,

H. Cazalé \& J. Vassal

(1956) Buli.d'Inform. au Comité Central a'oceanogr. et d'Etude des Cotes, 8 .

Gelci, R.

(1962) Journ.Mech.Phys. de I'Atmosph.,

2 Ser., $4,15-41$.

Grant, H.L.,

Stewart, R.W. \& Noilliet, A.

(1962) Journ.Fluia Mech. 12, 241-263.

Groves, G.W.

\& Melcer, J. (1961) Geofis.Intern. 1, 77-93. 


\begin{tabular}{|c|c|c|}
\hline Hasselmann, $K$. & $(1960)$ & Schiffstechnik 1, 191-195. \\
\hline Hasselmann, $K$. & (1962) & Journ. Fluia Mech. 12, 481-500. \\
\hline Hasselmann, $\mathrm{K}$. & $(1963 a)$ & Journ. Fluid Mech. 15, 273-281. \\
\hline Hasselmann, $K$. & $(1963 b)$ & Journ. Fluid Mech, 15, 385-398. \\
\hline Has selmann, $K$. & $(1966)$ & Rev. Geophys. $4,1-32$ \\
\hline Hasselmann, $K$. & $(1967 a)$ & Proc.Roy.Soc.A. (in the press). \\
\hline Hasselmann, $K$ & $(1967 \mathrm{~b})$ & (in preparation). \\
\hline
\end{tabular}

Hasselmann, $K_{\text {, }}$

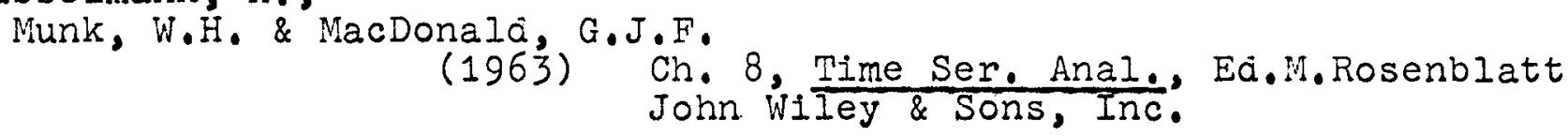

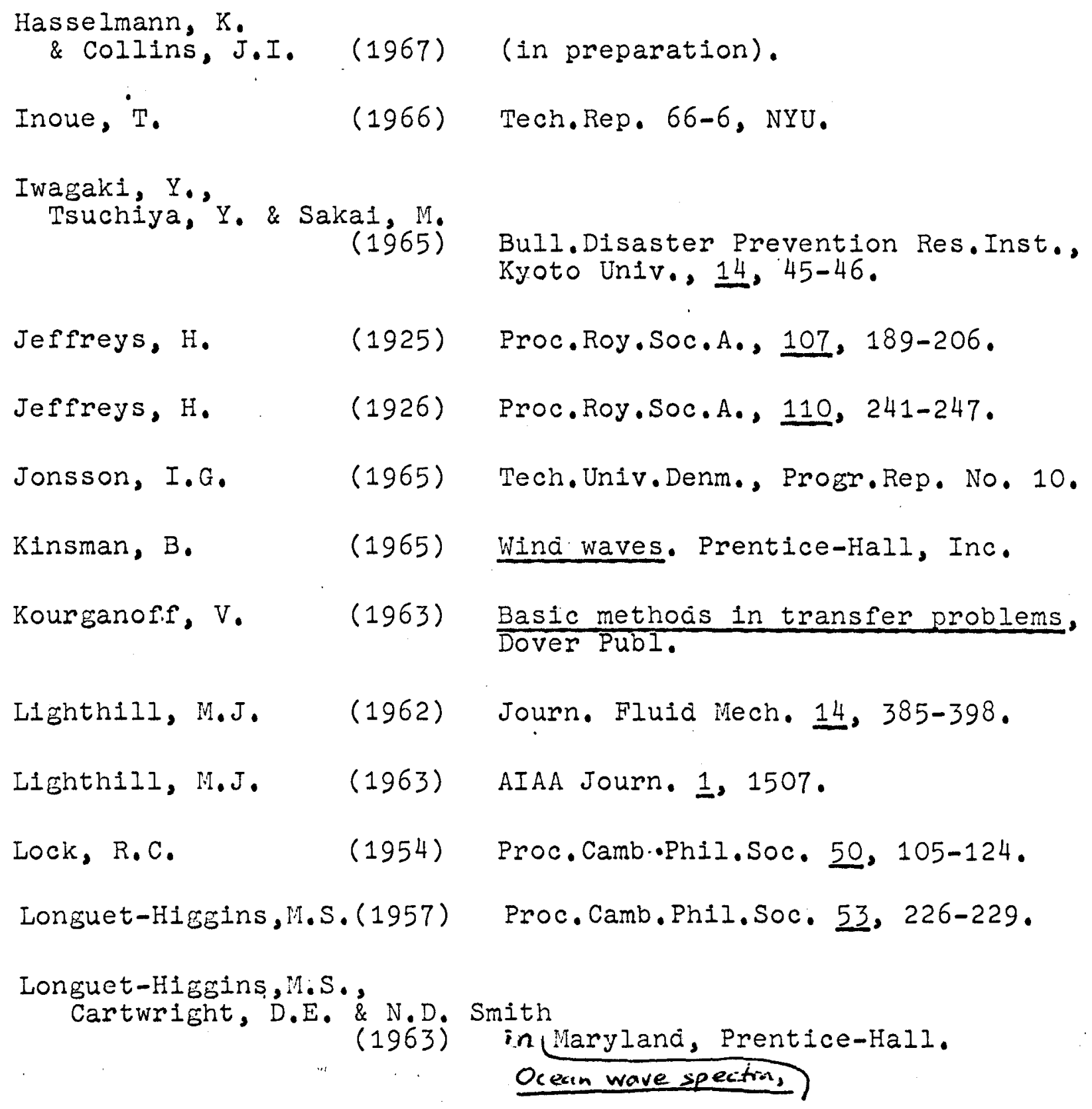




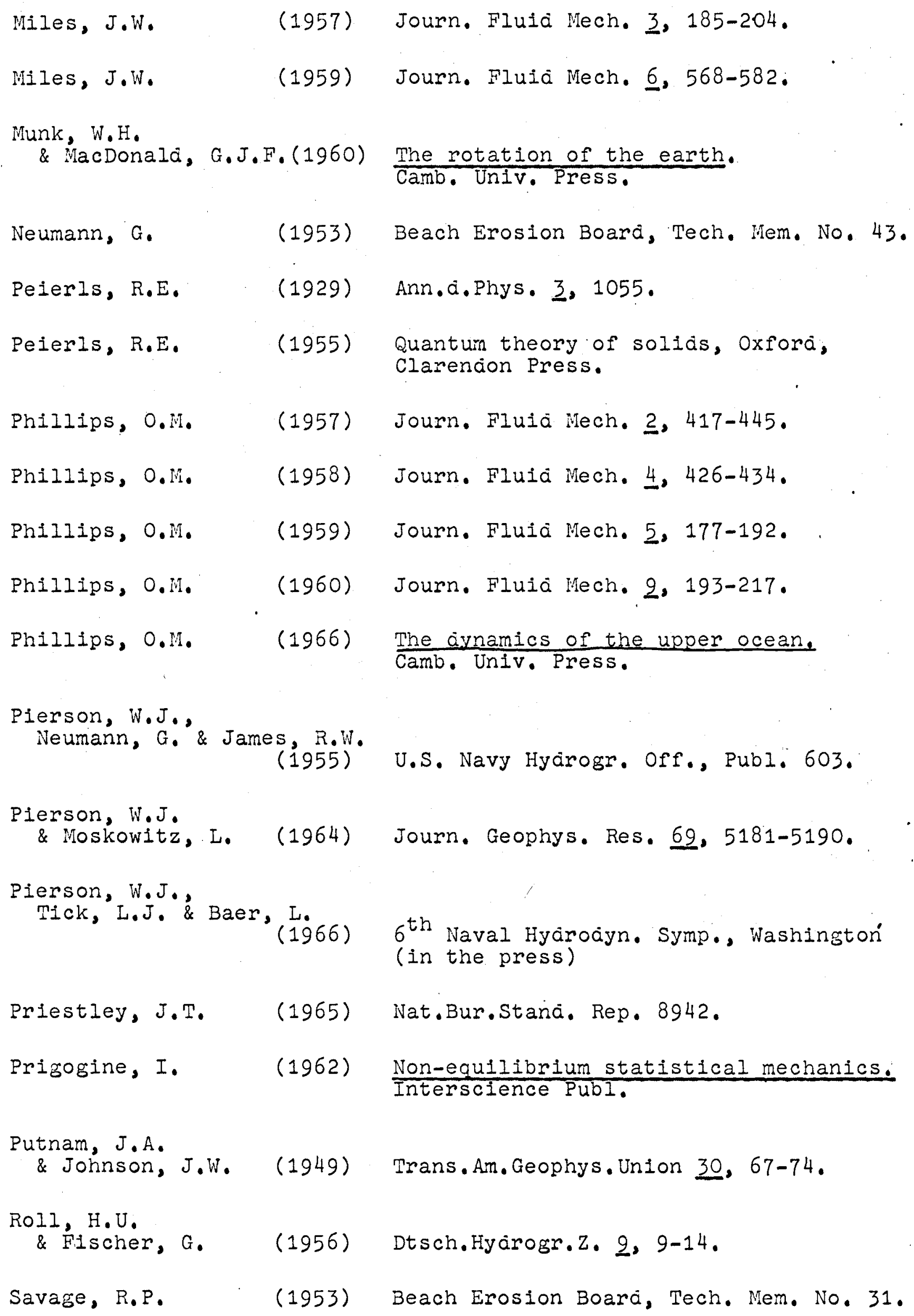




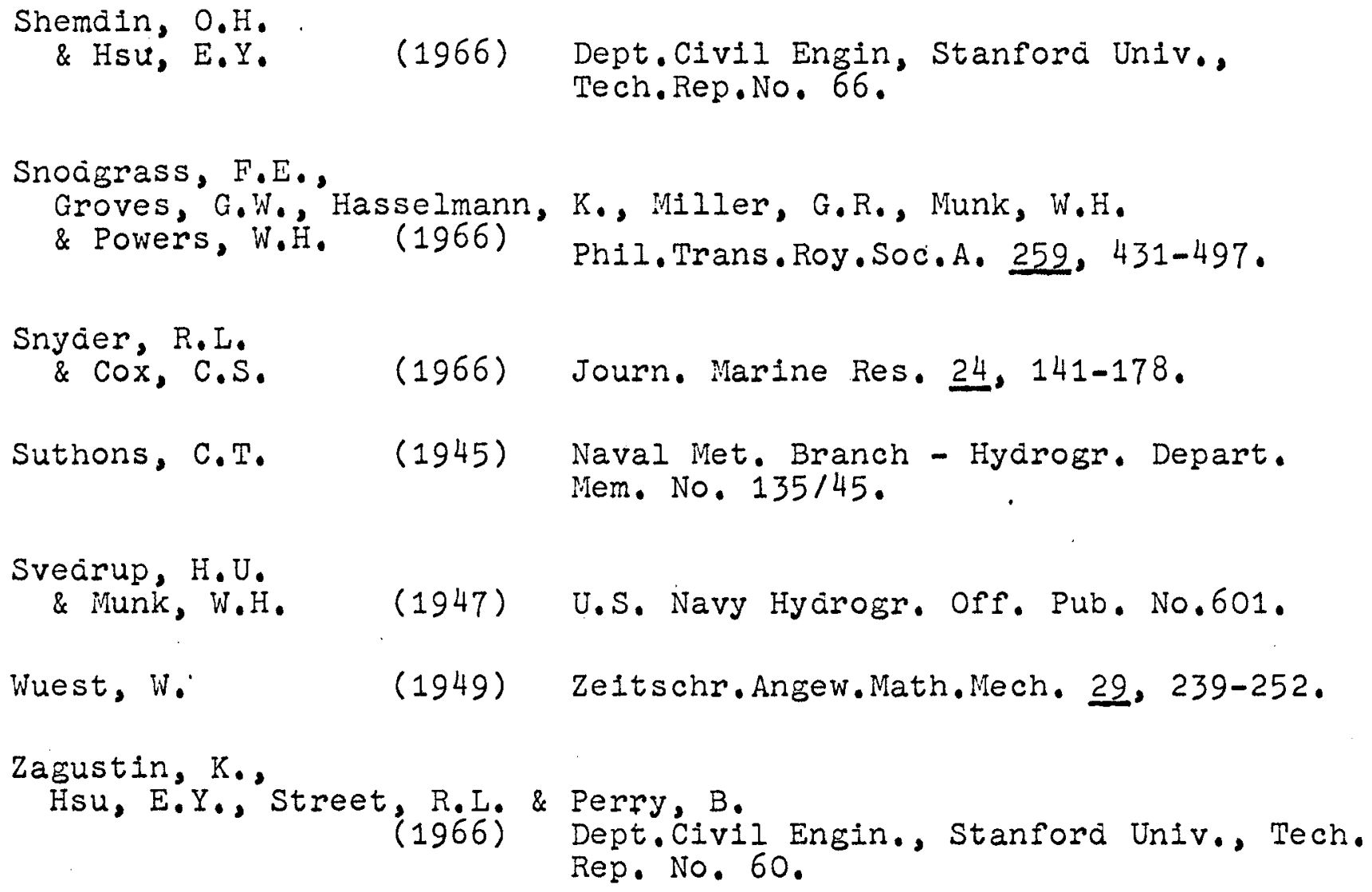


Fig. 1 Interaction diagrams corresponding to the linear transfer expressions (3.4.2) (diagrams (i)) and (3.4.3)

- (diagrams (ii))

Fig. 2 Interaction diagrams corresponaing to the source function (3.4.7). Diagrams (i), (ii) and (iii) correspond to the transfer functions $T_{\nu, \nu_{2} \rightarrow \nu}^{\nu}, T_{\nu \bar{\nu}_{1} \rightarrow \nu_{2}}^{\nu}$ and $T_{\nu \bar{\nu}_{2} \rightarrow \nu_{1}}^{\nu}$, respectively.

Fig. 3 Interaction diagrams corresponding to the source function (3.4.19). Diagrams (i) yield the first term of the transfer function $T_{\nu / \mu}^{\nu}$, equation (3.4.23). Diagrams (ii) correspond to the secona of the four terms in the parentheses $\{\ldots .$.$\} . The remaining terms in the parentheses are$ obtained by interchanging components and anti-components.

Fig. 4 Transfer aiagrams corresponaing to the transfer ex-

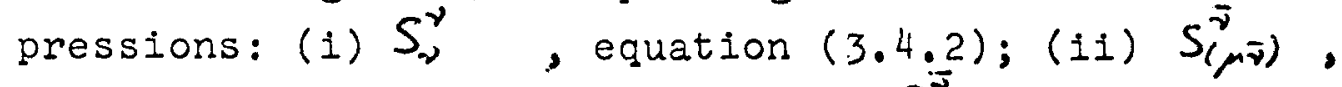
equation $(3.4 .3)$; (iii), (iv), (v) $S_{\left(\nu, v_{2} \bar{\nu}\right)}^{\bar{\nu}}$, equation $(3.4 .7) ;(v i) S_{\nu \nu_{1}}^{\nu}$, equation $(3.4 .13)$. Diagrams (ii) - (v) represent scattering aiagrams, (i) and (vi) parametric diagrams. Diagrams (iii), (iv) and (v) belong to a alagram set.

Fig. 5 Interaction (Feynman) diagrams for conservative gravitywave interactions. The energy transfer is given by equation $(3,6.5)$. The cormesponding transfer aiagrams are of the same $\sqrt{a s}$ aiagram (iii).

Fig. 6 . Lowest-oräer transfer diagrams for wave-atmosphere ana wave-ocean interactions. Diagrams (i), (ii) and (v) represent parametric processes, aiagrams (iii) and (iv) scattering processes. 
Wave-atmosphere interactions (i) : Iinear interactions with the ocean bounaary-layer flow according to Miles, (ii) : non-linear interaction with mean bounaary-layer flow, (iii) : Eckart-Phillips wave generation by turbulence. C

The process can be representea by three diagrams in terms of the turbulent velocity components $t$ or an equivalent Iinear aiagram $p^{t} \rightarrow \delta$, where $p^{t}$ is the turbulence pressure at the surface. (iv), (v) : wave-turbulence interactions.

Wave-ocean interactions (i) : linear interaction with mean current. The energy transfer vanishes in the weak-interaction approximation. (ii) : non-linear interactions with mean currents,(iii) : generation of waves by turbulence, (iv) : scattering of waves by turbulence, (v) : parametric aamping of waves by turbulence ("edày viscosity").

Fig. 7 Spectral distribution of surface pressure for waveatmosphere interactions (schematic). Shaing represents aistribution of the three-aimensional spectral aensity $F_{p}(k, \omega)$ in the $k_{1}-\omega$ plane.

Fig. 8 The growth parameter $\beta$ as function of wind speed $U$. $J$ : Jeffreys (1925), MI : Miles, BF : best-fit experimentally; $T$ : an empirical relation $\beta=\frac{\rho_{a}}{\rho}(k \cdot \mu-\sigma)$ suggested by Snyäer ana Cox (1966); The aata points are äiviaed into short runs + anä long runs $\square$. (From Snyäer anà Cox, 1966).

Fig. 9 Non-linear interaction äiagram for parametric damping of waves by mean currents.

Fig. 10 Interaction aiagrams for parametric äamping of waves by turbulence ("eäà viscosity").

Fig. 11 Wave damping aue to a turbulent bottom friction $\tau=-\rho c_{p} \mu|u|$, with $c_{f}=0.015$. The initial aistribution corresponas to a 40-knot Pierson-Moskowitz spectrum with a $\cos ^{4} \phi$ spreading factor. The water depth is $100 \mathrm{~m}$. (From Hasselnann ana Collins, 1967). 
Fig. 12 Wave camping aue to turbulent bottom friction. The same case as in fig. 11, with a superimposea bottom current of $0.7 \mathrm{~m} / \mathrm{sec}$ at $45^{\circ}$ to the initial mean wave direction. (From Hasselmann anà Collins, 1967).

Fig. 13 Wave spectra (upper panels) and the computed energy transfer due to wave-wave scattering (lower panels) in the near zone of a storm. Full ana dashea curves in the lower right panel correspona to a $\cos ^{4} \varphi$ and $30^{\circ}$ step-function spreaaing factor, respectively. (From snoagrass et al, 1966). 


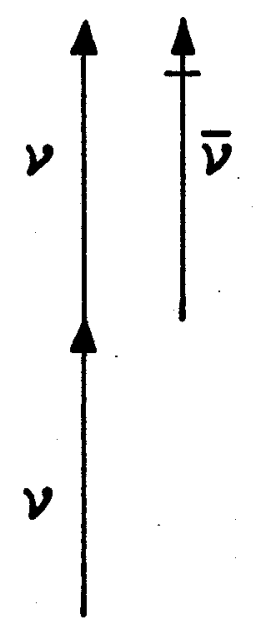

(i)

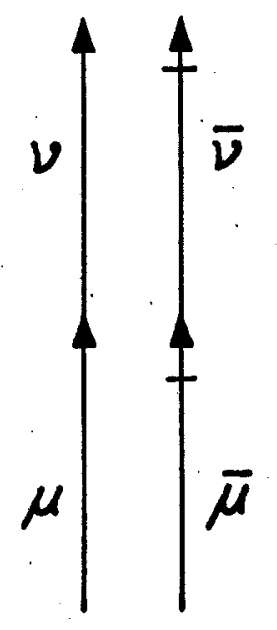

(ii)

Fig. 1 


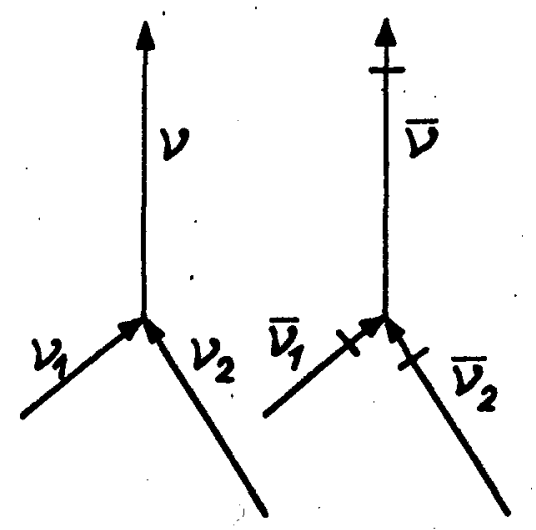

(i)

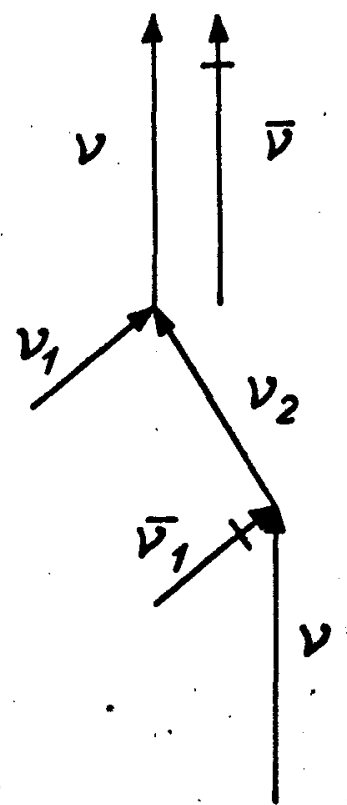

(ii)

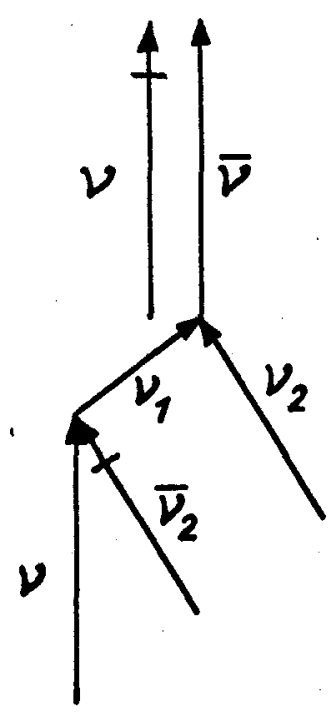

(iii)

Fig. 2 


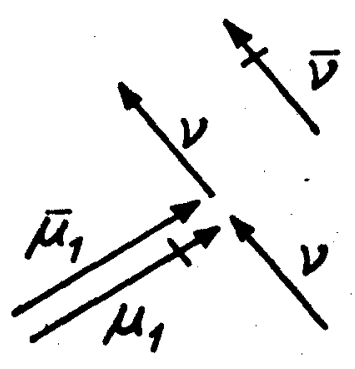

(i)

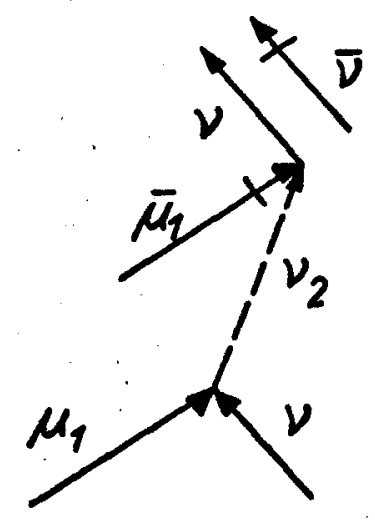

(ii)

Fig. 3 


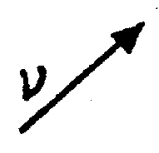

(i)

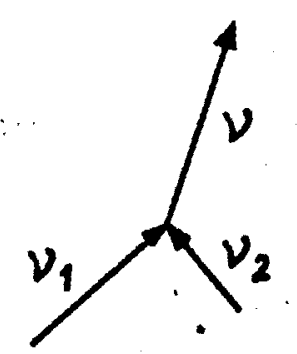

(iii)

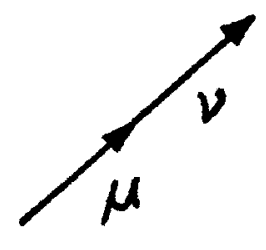

(ii)

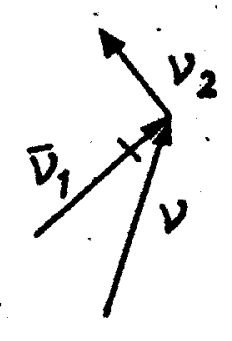

(iv)

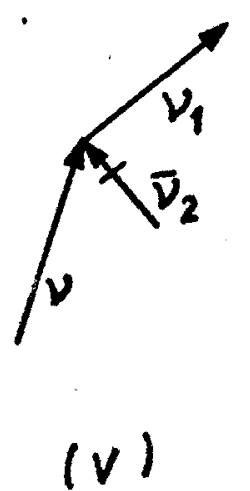

(v)

$$
\begin{aligned}
& \mu / \nu \\
& (v i)
\end{aligned}
$$

Fig. 4 


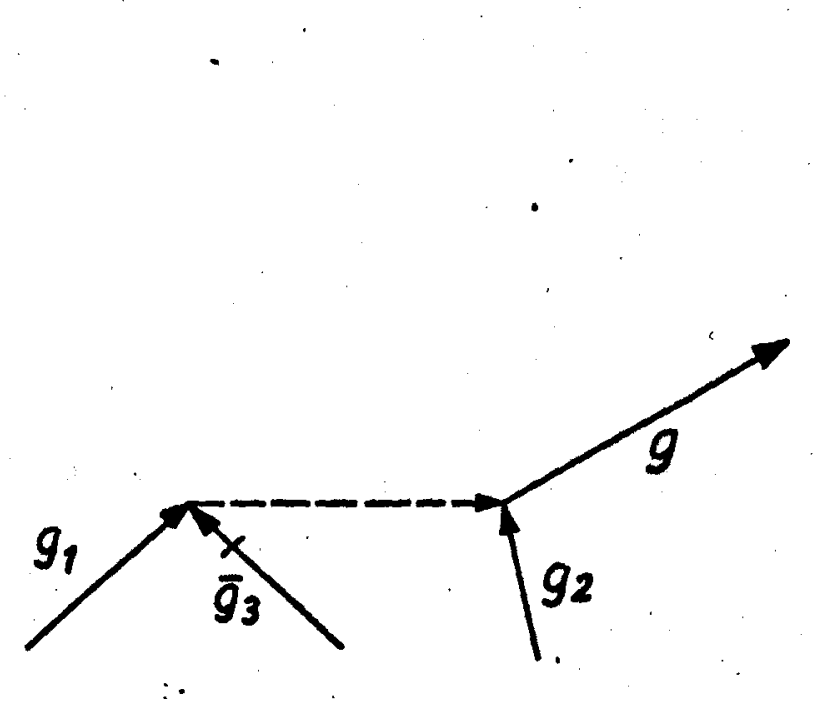

(i)

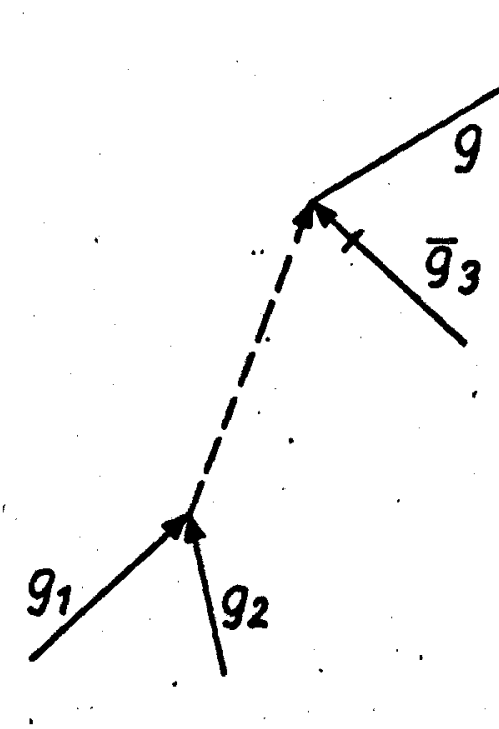

(ii)

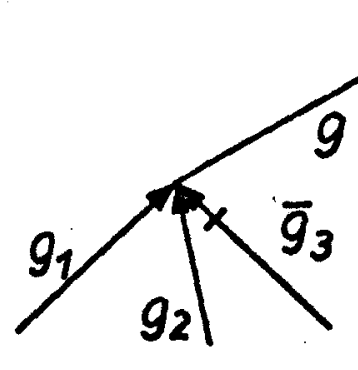

(iii)

Fig. 5 


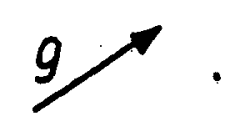

(i)

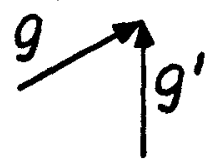

(ii)
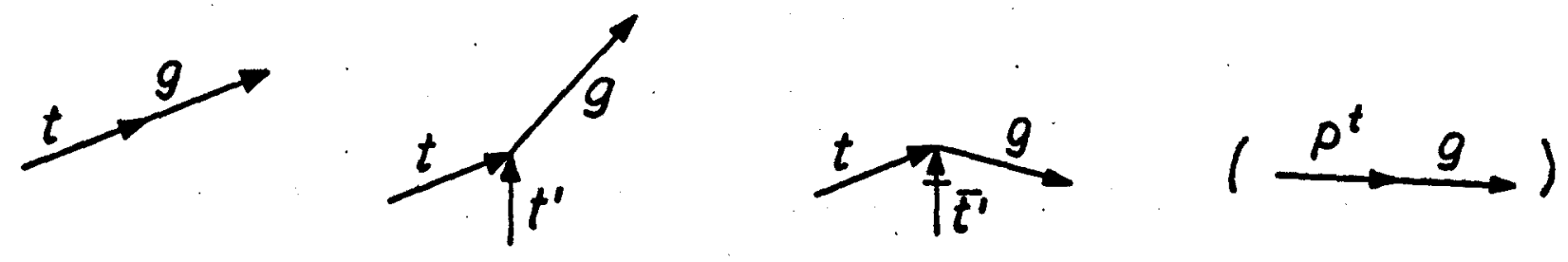

\section{(iii)}
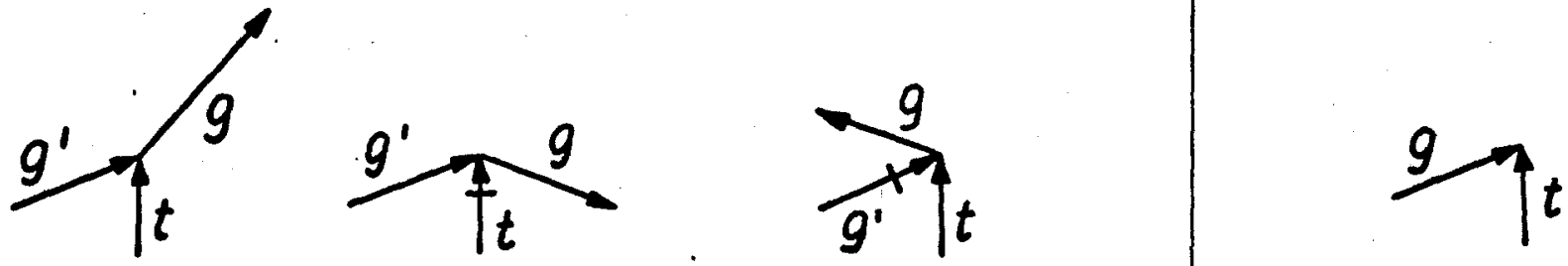

(iv)

(v)

Fig. 6 


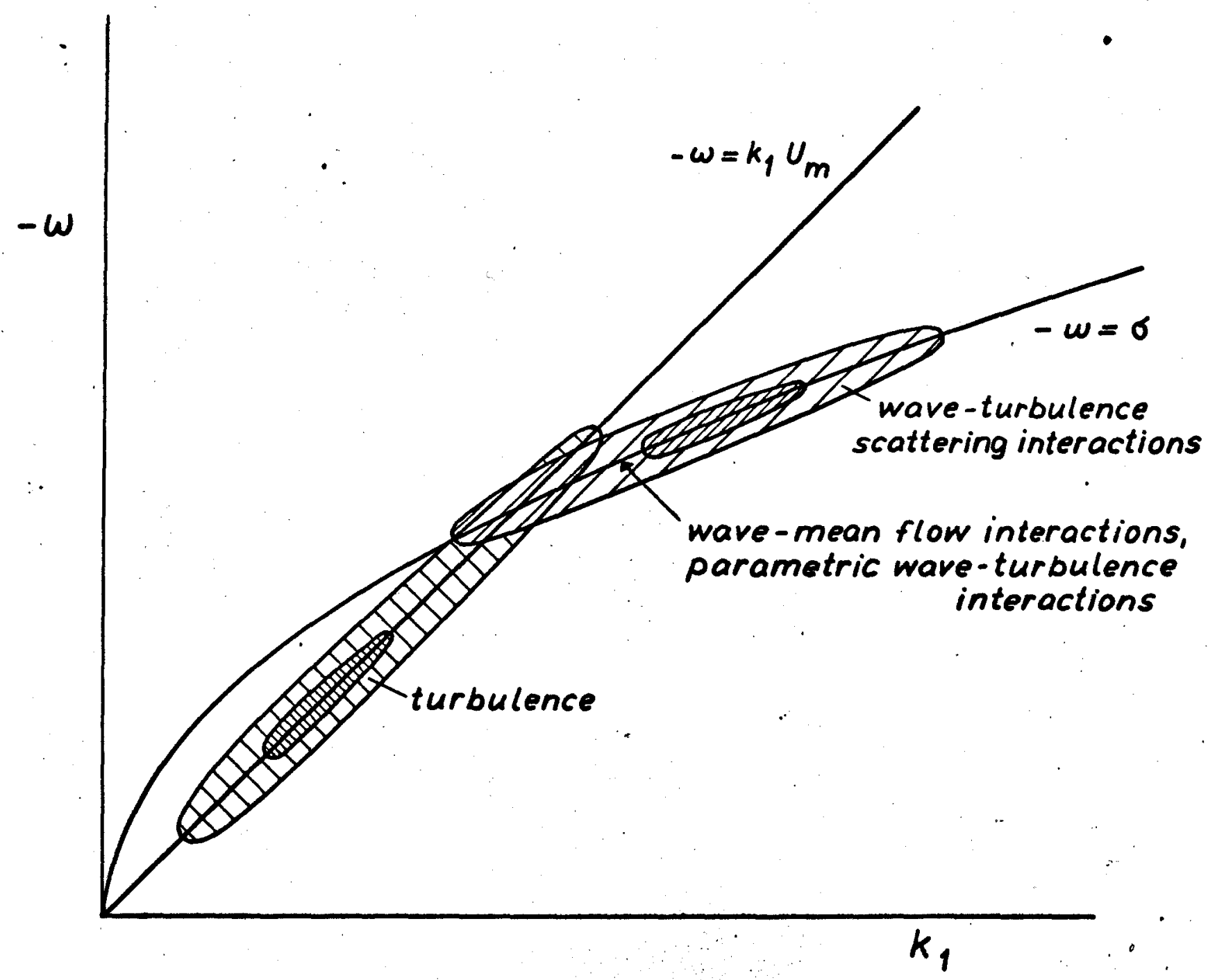

Fig. 7 


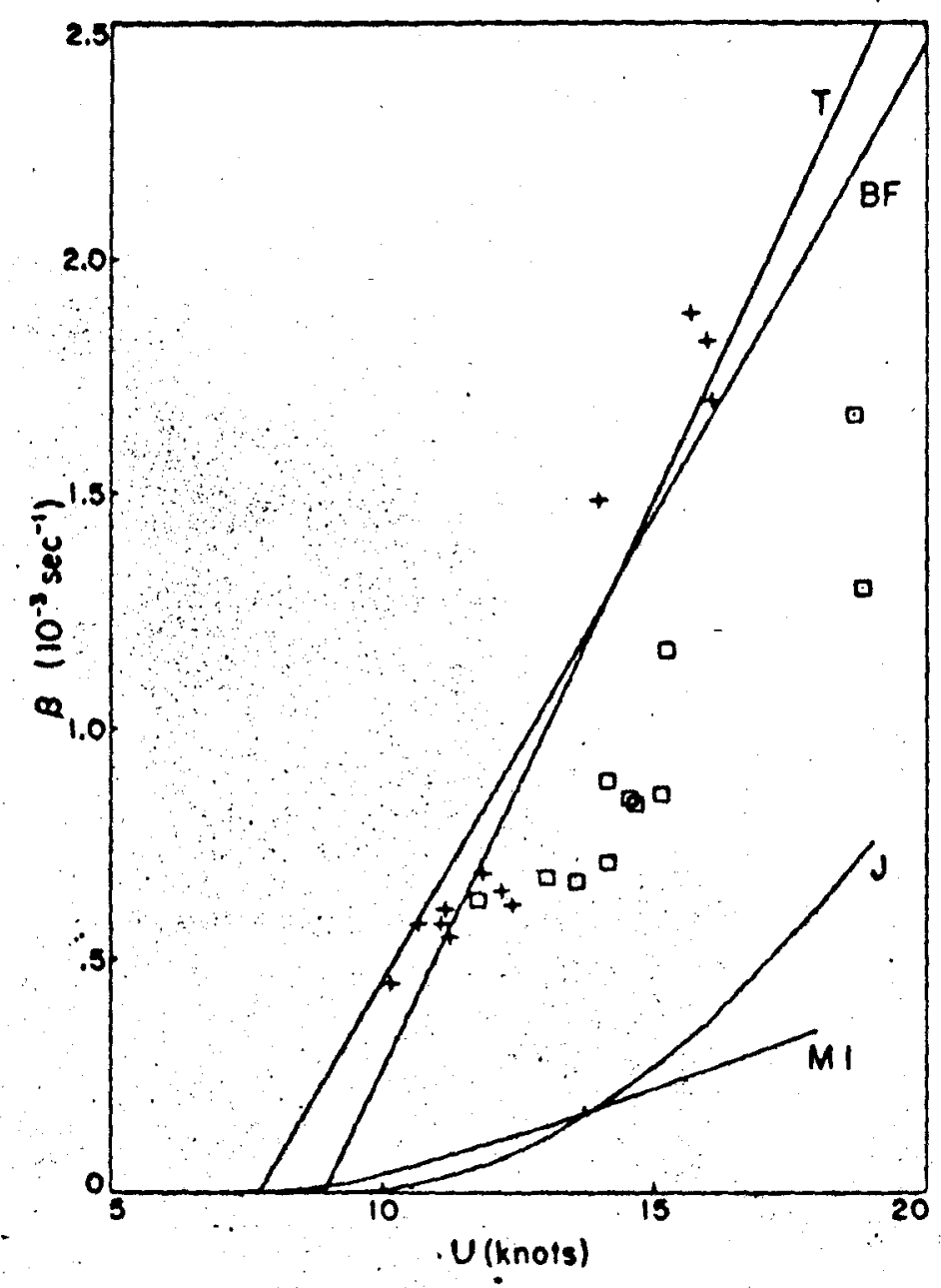

Fig. 8 


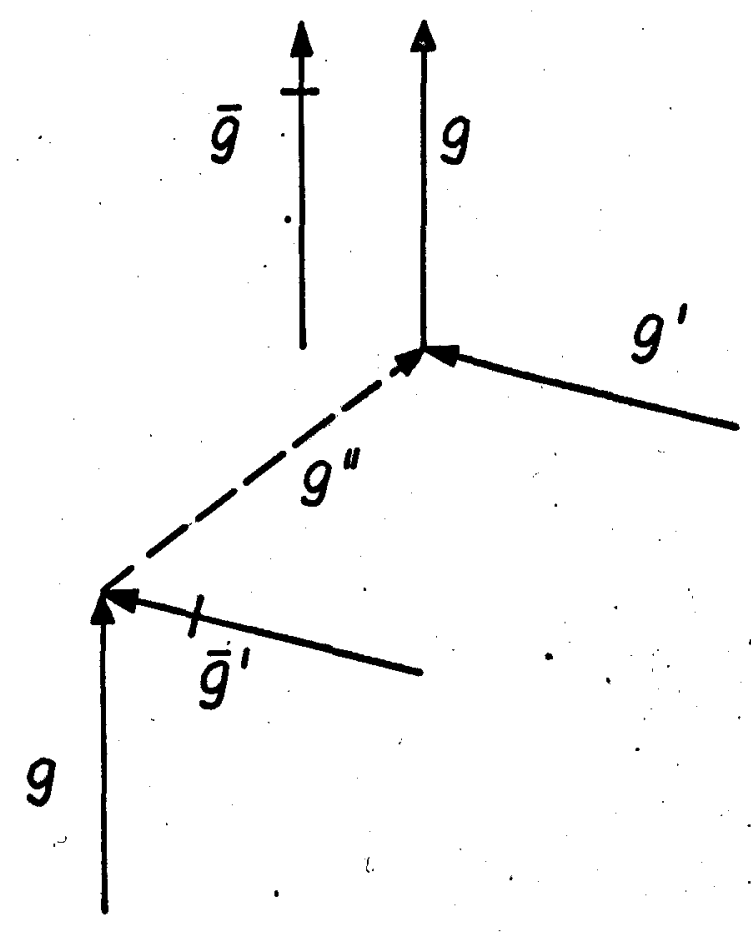

Fig. 9 


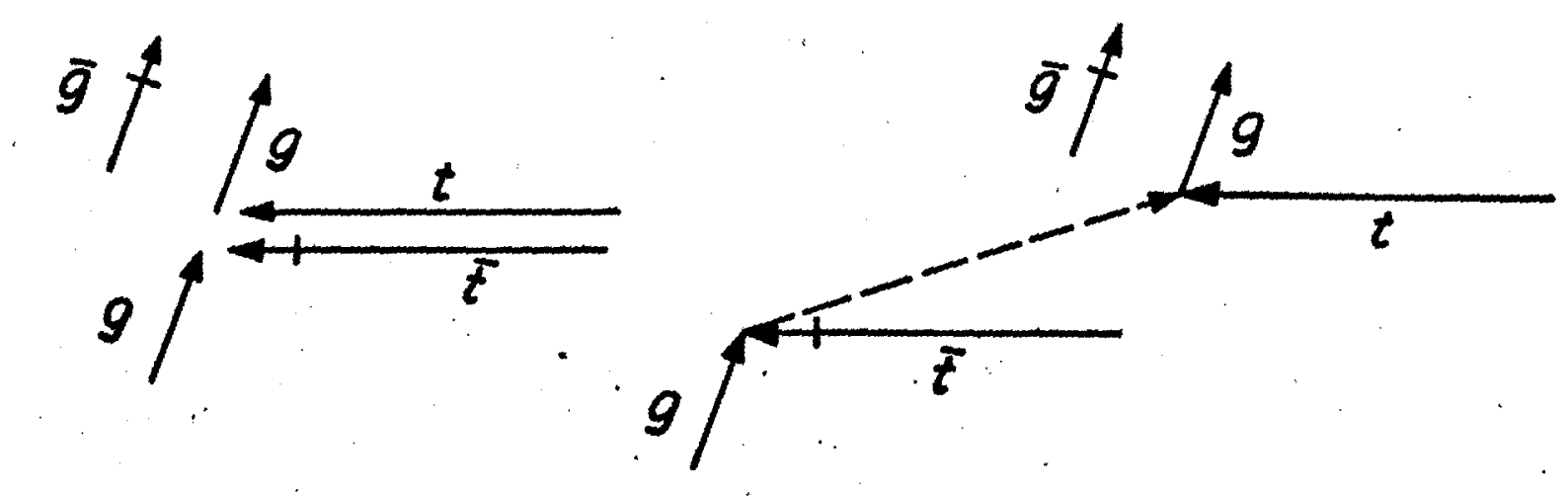

Fig. 10 


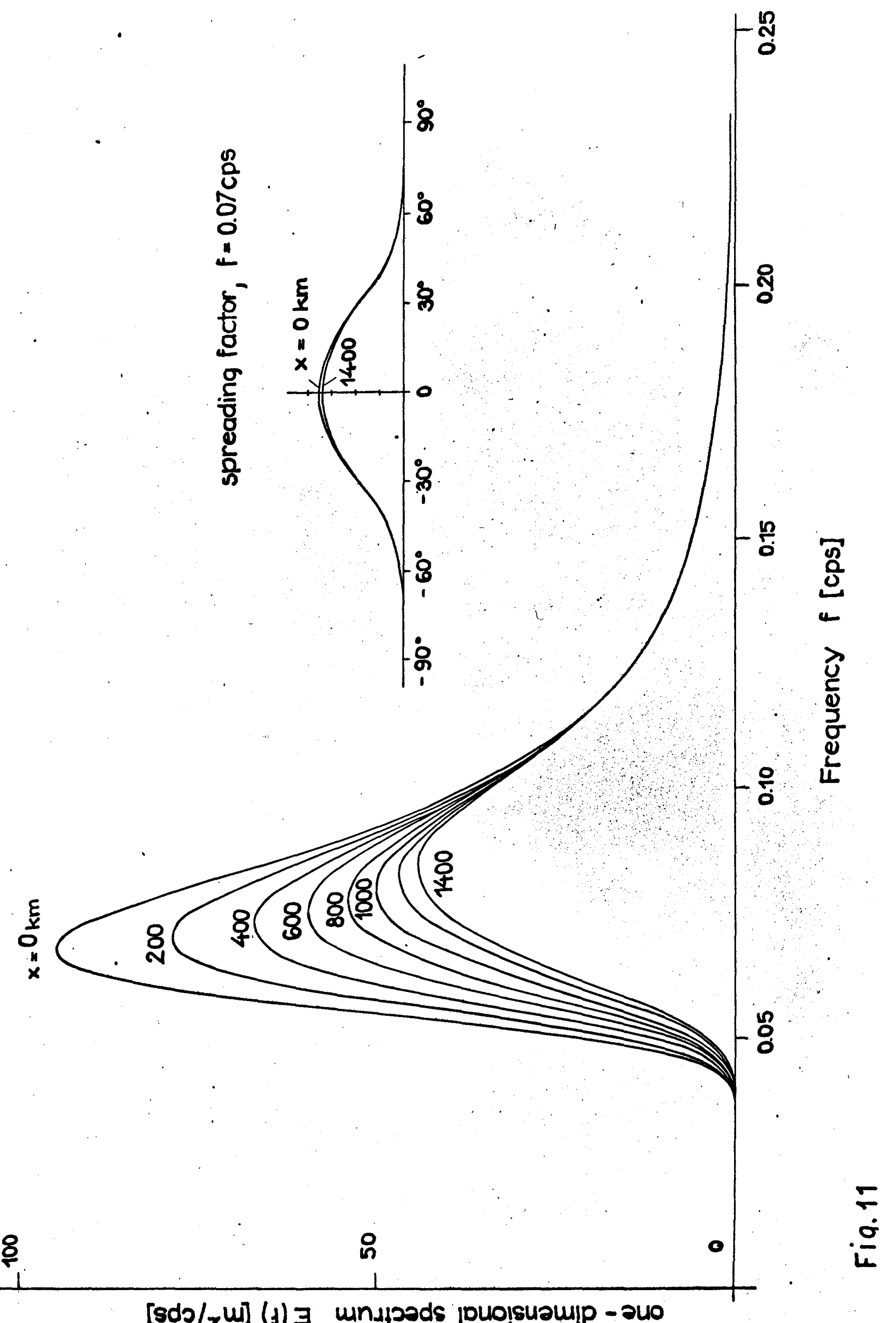




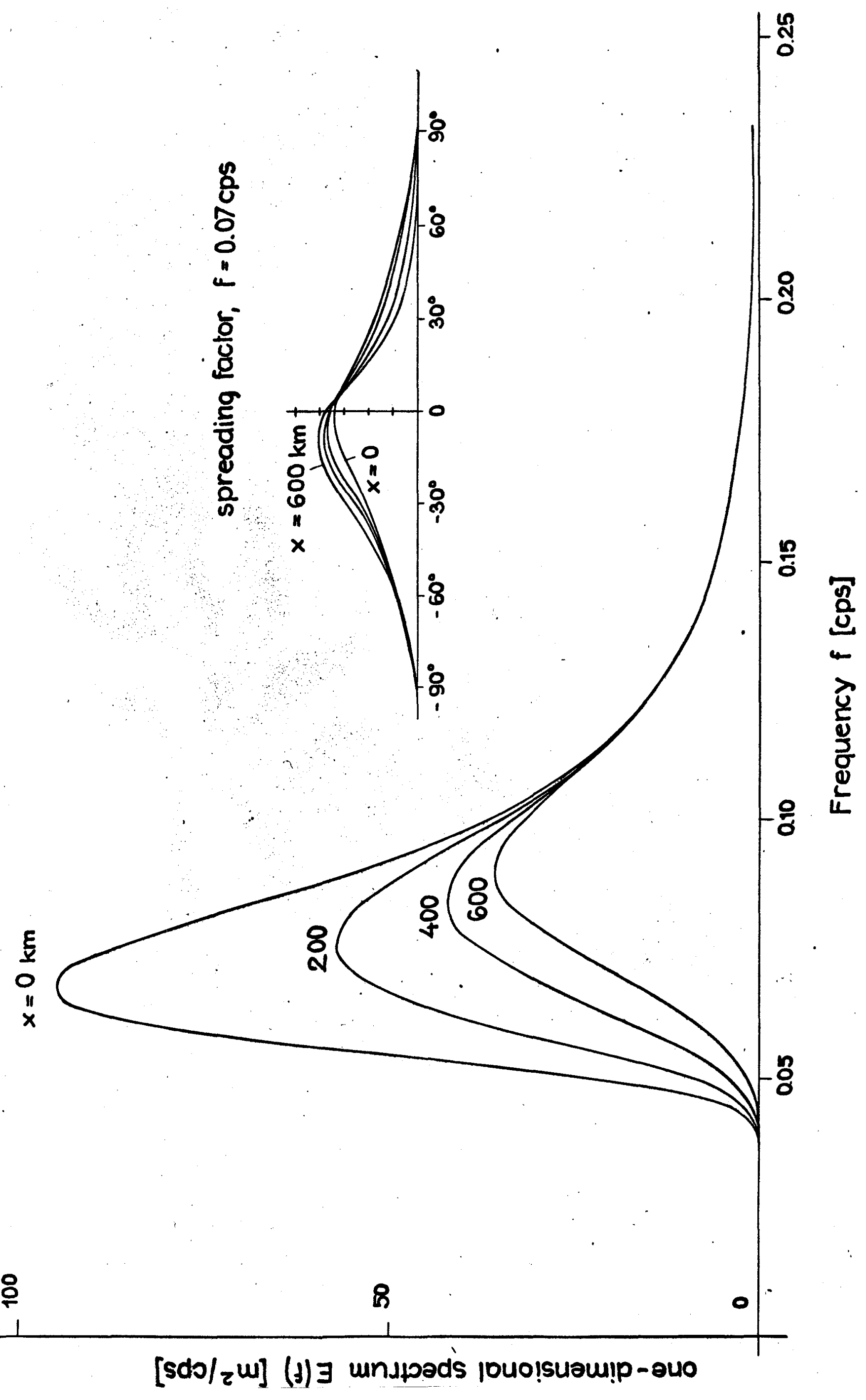



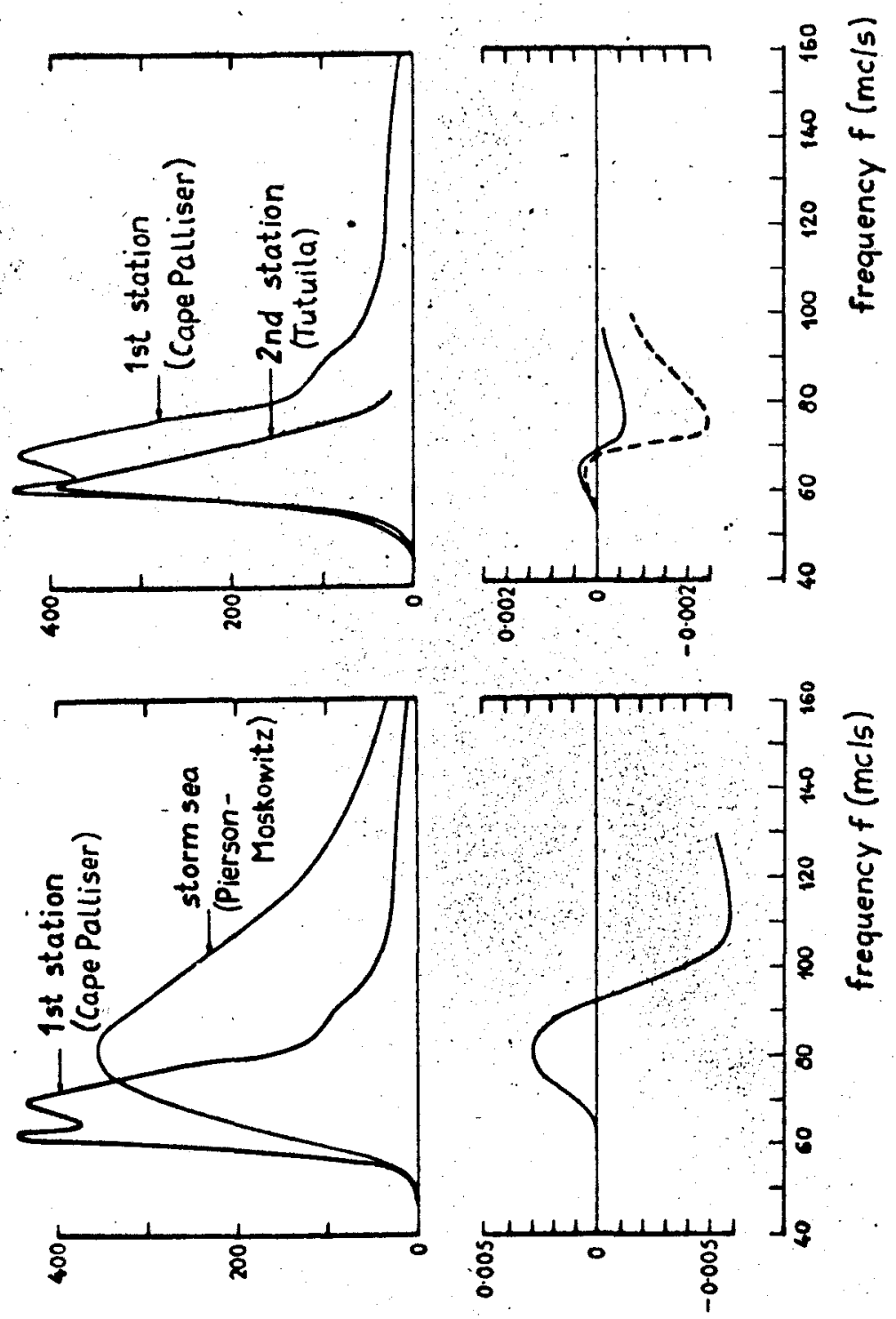

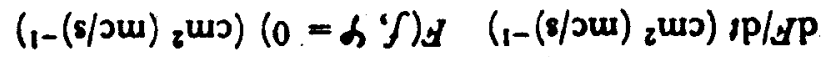

Fig. 13 